\title{
Zooming into the micro-level of experience : an approach for understanding and treating psychopathology
}

Citation for published version (APA):

Kramer, I. M. A. (2015). Zooming into the micro-level of experience : an approach for understanding and treating psychopathology. [Doctoral Thesis, Maastricht University]. Maastricht University. https://doi.org/10.26481/dis.20150430ik

Document status and date:

Published: 01/01/2015

DOI:

10.26481/dis.20150430ik

Document Version:

Publisher's PDF, also known as Version of record

\section{Please check the document version of this publication:}

- A submitted manuscript is the version of the article upon submission and before peer-review. There can be important differences between the submitted version and the official published version of record.

People interested in the research are advised to contact the author for the final version of the publication, or visit the DOI to the publisher's website.

- The final author version and the galley proof are versions of the publication after peer review.

- The final published version features the final layout of the paper including the volume, issue and page numbers.

Link to publication

\footnotetext{
General rights rights.

- You may freely distribute the URL identifying the publication in the public portal. please follow below link for the End User Agreement:

www.umlib.nl/taverne-license

Take down policy

If you believe that this document breaches copyright please contact us at:

repository@maastrichtuniversity.nl

providing details and we will investigate your claim.
}

Copyright and moral rights for the publications made accessible in the public portal are retained by the authors and/or other copyright owners and it is a condition of accessing publications that users recognise and abide by the legal requirements associated with these

- Users may download and print one copy of any publication from the public portal for the purpose of private study or research.

- You may not further distribute the material or use it for any profit-making activity or commercial gain

If the publication is distributed under the terms of Article $25 \mathrm{fa}$ of the Dutch Copyright Act, indicated by the "Taverne" license above, 


\section{Zooming into the micro-level of experience}

An approach for understanding and treating psychopathology

Ingrid Kramer 
ISBN 978-94-6299-064-7

Layout and printing: Ridderprint BV, Ridderkerk, the Netherlands Cover: Ridderprint BV, Ridderkerk, the Netherlands

(C) 2015 | Ingrid Kramer

All rights reserved. No part of this publication may be reproduced, stored in a retrieval system, or transmitted, in any form or by any means, mechanically, electronically, by photocopy, by recording or otherwise, without prior written permission of the author. 


\title{
Zooming into the micro-level of experience
}

\author{
An approach for understanding \\ and treating psychopathology
}

\author{
PROEFSCHRIFT
}

Ter verkrijging van de graad doctor aan de Universiteit Maastricht, op gezag van de Rector Magnificus, Prof. dr. L.L.G. Soete, volgens het besluit van het College van Decanen, in het openbaar te verdedigen op donderdag 30 april 2015 om 10:00 uur

door

\section{Ingrid Maria Adriana Kramer}

geboren op 12 april 1973 te Sittard 


\section{Promotor}

Prof. dr. J. van Os

\section{Copromotores}

Dr. M. Wichers

Dr. C.J.P. Simons

\section{Beoordelingscommissie}

Prof. dr. M.W. de Vries (voorzitter)

Prof. dr. W.J.M.J. Cuijpers (Vrije Universiteit Amsterdam)

Prof. dr. P.N. van Harten

Prof. dr. L. Krabbendam (Vrije Universiteit Amsterdam)

Dr. M. Marcelis

The research presented in his thesis was performed at the School for Mental Health and Neuroscience (MHeNS), Department of Psychiatry and Psychology, Maastricht University, Maastricht, the Netherlands.

The publication of this thesis was financially supported by: Servier Nederland Farma B.V., Janssen-Cilag B.V., Eurocept Pharmaceuticals 
Voor Floris, Maurits en het kleintje in mijn buik 
Paranimfen

Evert Krämer

Johan Pluijmakers 


\section{Table of contents}

Chapter 1 General introduction

Part 1 Experience Sampling Method for understanding psychopathology

Chapter 2 Evidence that genes for depression impact on the pathway from trauma 21 to psychotic-like symptoms by occasioning emotional dysregulation

Chapter 3 Time-lagged moment-to-moment interplay between negative affect and paranoia: new insights in the affective pathway to psychosis

\section{Part 2 Experience Sampling Method for treating psychopathology}

Chapter 4 Momentary assessment technology as a tool to help patients with depression help themselves

Chapter 5 A therapeutic application of the experience sampling method in the treatment of depression: a randomized controlled trial

Chapter 6 General Discussion

Chapter 7 Valorization, clinical implications and future directions

Epilogue Summary

Samenvatting

Dankwoord

Curriculum Vitae

List of publications 


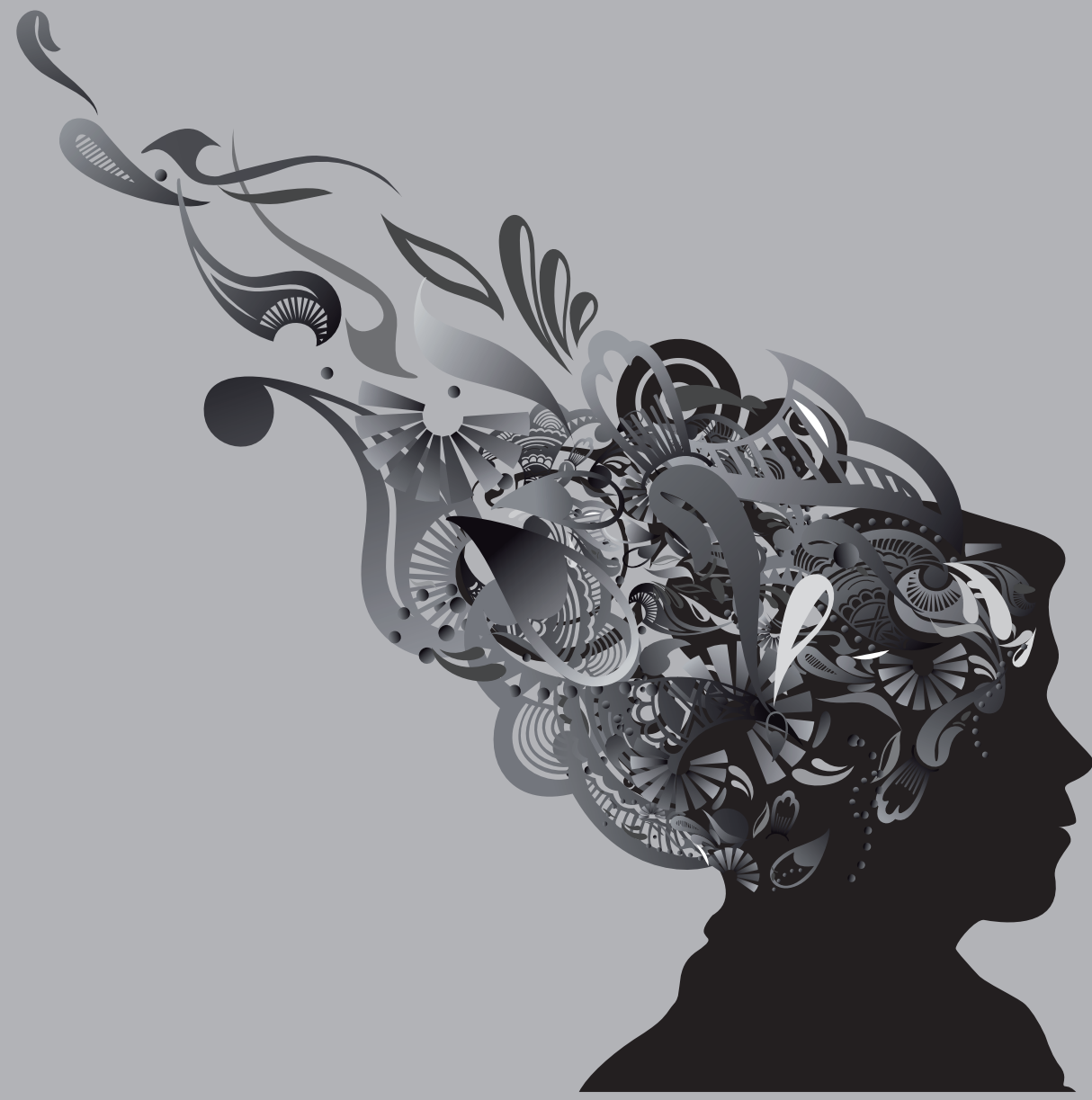




\section{CHAPTER 1}

General Introduction 
Mental disorders are common. The Netherlands Mental Health Survey and Incidence Study (NEMESIS) demonstrated that in the Netherlands $41.2 \%$ of the respondents experienced at least one mental disorder during their life (1). Symptoms of mental disorders interfere in the daily life of the individual and may create a substantial burden to the individual. They may be so overwhelming that the individual is less able or unable to fulfill daily roles and activities. Therefore, mental disorders are an important cause of loss of productivity and societal costs (2). Two dimensions of psychopathology associated with a substantial burden, are major depression and psychosis.

Major depression is a common mental disorder (life-time prevalence of $15.4 \%$ in the Netherlands (1)) characterized by feelings of sadness and/or a loss of interest or pleasure in activities almost every day for most of the days. If this occurs for at least two weeks and five or more additional symptoms are present, criteria for a DSMIV major depressive episode are fulfilled (3). Accompanying symptoms of a major depression are suicidal ideation, feelings of guilt or worthlessness, significant weight loss or gain, loss of energy, diminished ability to think or concentrate, and sleep disturbances (insomnia or hypersomnia). Growing evidence indicates that depression is a continuous phenotype ranging from the more prevalent subclinical or subthreshold depression, such as experiencing a low mood and/or loss of interest, to severe DSM-IV major depression at the other end (4-7). The depression continuum is associated with significant psychological distress, substantial loss of quality of life, disability in daily life activities, and high societal costs due to increased use of health services, decreased employment and productivity (4, 7-9). According to the WHO, by 2020, depression will be the second leading illness in terms of global burden of disease (6).

Non-affective psychosis (hereafter psychosis), on the other hand, is a less common but also potentially very disabling mental disorder (10). It is characterized by the presence of at least two of the following symptoms: delusions, hallucinations, disorganized speech, catatonic behavior or negative symptoms with a duration of at least one month, which cause occupational and social dysfunction for at least 6 months (3). Mounting evidence indicates that, analogue to the depression continuum, psychosis is a continuous phenotype that ranges from the more prevalent subclinical level of expression to the clinical phenotype with severe symptoms leading to impairment and help-seeking behavior (11-16). General population data of the Netherlands indicate a high life time prevalence of any psychotic or psychotic-like experience of $17.5 \%$ (11) while the lifetime prevalence of the clinical DSM-IV psychosis is much lower $(0.4 \%$ (1)).

Given the burden and large societal costs resulting from both depression and psychosis, it is important to gain more insight in the developmental pathways of the depression and psychosis continuum, as well as to develop effective interventions to treat these disabling psychiatric conditions. A promising way to examine the depression 
and psychosis continuum is to shift from the macro-level of affective and psychotic symptomatology to the micro-level of the 'smallest building blocks' of emotional experiences. This approach may lead to new insights in developmental pathways that could also contribute to the development of effective interventions.

The present thesis starts with (i) an examination of affective factors in the development of psychosis on a macro-level of symptomatology. To further unravel this developmental pathway (ii) a shift to the micro-level of daily life emotional experiences will be made. Continuing on the micro-level, it will be explored (iii) whether the microlevel of daily life emotional experiences may contribute to a refinement of the diagnostic process and the effectiveness of treatment of mental disorders.

\section{Affective pathway to psychosis}

Rising evidence indicates that on a macro-level of symptomatology, depression and psychosis often co-occur (17-20). Depression and psychosis tend to cluster in the same individual (21-23) as well as in the same families (24-26), suggesting shared genetic liability. Likewise, several environmental risk factors that are associated with the development of depression are also linked to the development of psychosis. Examples are childhood trauma (27-29), stress sensitivity (30-33), social defeat $(34,35)$, and negative life events (36-38). Given the frequent co-occurrence and shared environmental and genetic risk factors for the depression and psychosis continuum, it is attractive to hypothesize that there may be a shared developmental pathway. This is accounted for in the model of Myin-Germeys and van Os (39). They presented an affective pathway to psychosis in which childhood adversity and life events were identified as risk factors that result in an increased sensitivity to daily life stress, which sets an individual at risk for the development of psychosis. In chapter 2 , the affective pathway to psychosis was further explored on a macro-level of symptomatology.

To further unravel the relationship between affective and psychotic experiences and the impact of putative risk factors, a shift from the macro-level of symptomatology to the micro-level of daily life emotional experiences may help to elucidate underlying mechanisms. Zooming into daily life individual momentary emotional experiences may give a more refined and fine-grained view on the dynamic nature of depressive and psychotic experiences, the context in which they arise or react to, and underlying mechanisms. This approach calls for a measurement technique that is able to provide fine-grained, high resolution assessments on the micro-level of daily life emotional experiences.

\section{Experience Sampling Method}

The Experience Sampling Method (ESM) is ideally suited to capture subtle, momentary 
emotional experiences and dynamics in the flow of daily life. It is a within-day, momentary assessment technique that has been used to examine moment-tomoment emotional reactions to stress $(30,40-43)$. ESM provides the opportunity to assess current context, mood, thoughts, and symptoms in the flow of one's daily life. It encompasses frequent assessments resulting in a fine-grained film of daily life emotional experiences embedded in their context. ESM results in prospective, repetitive momentary assessments that are, in contrast to retrospective laboratory or in-the-office measurements, ecological valid and not influenced by emotional and cognitive biases $(44,45)$. For example, momentary assessments of dynamic daily life emotional patterns improve, independent of symptom severity assessed by retrospective measurements, the prediction of future symptomatology in patients with a history of major depression (46). The prospective, longitudinal character of the assessments makes it also possible to examine temporal dynamics of the variables of interest $(44,45)$. Recent ESM work has, for example, demonstrated that momentary emotional experiences such as an increase in anxiety predict the onset of a paranoid episode (47).

In the last three decades of ESM research, subjects received a digital wristwatch and a set of ESM self-assessment forms collected in a booklet for each day. Current technology, however, provides the possibility to obtain electronic or digital ESM assessments by means of a PDA or mobile phone app. In general, the ESM device (PDA, mobile phone, or wristwatch) is programmed to emit a signal (beep) at ten unpredictable moments a day in each of ten 90-minute time blocks between 7:30 and 22:30, and subjects are asked immediately after the beep to report their thought, current context, appraisals of the current situation, and mood $(30,32)$.

Using the Experience Sampling Method, the 'film of daily life' can be captured resulting in the possibility to zoom into the micro-level of emotional experiences and examine temporal dynamics between symptom clusters to further unravel developmental pathways of mental disorders. In chapter 3, ESM was used to further explore the affective pathway to psychosis on a micro-level of daily life emotional experiences.

\section{From science to practice}

Until recently, ESM or momentary assessment has been used only in the context of research to identify moment-to-moment patterns and mechanisms of psychopathology. This has resulted in a large body of knowledge on etiology and phenomenology of mental disorders $(44,48)$ such as major depression $(27,30,31,46,49,50)$ and psychosis $(32$, 33). Outside the domain of mental health care, momentary assessment techniques are already used in the assessment and treatment of hypertension (51), diabetes mellitus (52) and Parkinson disease (53). Likewise, ESM may also contribute to the diagnostic process in mental health care. Repetitive, prospective, in-the moment assessments of 
mental health symptomatology embedded in its context may provide a more reliable view on actual symptomatology and its variation over time compared to the traditional in-the-office assessments that heavily rely on memory and hence are likely influenced by cognitive but also emotional biases (54). The value of repetitive assessments to refine the diagnostic process and the evaluation of treatment effect has been recognized in clinical practice as evidenced, for example, by the widely used life chart methodology used by bipolar patients to capture past and current course of symptomatology (55, 56). Given the wealth of detailed ecologically valid and reliable daily life information provided by ESM, this procedure may also be fruitful in mental health care to refine the diagnostic process and treatment of heterogeneous mental disorders, such as depression (45, 47, 57-59). Chapter 4 encompasses a literature review in which the potential contribution of ESM in diagnostics and treatment of major depression was examined.

As major depression is one of the world's leading causes of disability (6), it is very important to optimize treatment. Unfortunately, adequate psychological and/ or pharmacological treatment is only available for a minority of patients (60), and of those that receive adequate treatment a high percentage is treatment resistant (61). For example, in a large group of depressed patients receiving pharmacological treatment for a period of 12-14 weeks, full remission was only accomplished in a third of the patients (62). Therefore, it is very important to search for opportunities to improve treatment outcome. Self-monitoring of symptoms is a psychological intervention that has been demonstrated to decrease depressive symptomatology (63-65). This procedure generally comprises once a day retrospective assessments of mood. As ESM consists of prospective, repetitive in-the-moments assessments, ESM may not only enhance the reliability of the self-monitoring assessments, but may also lead to the possibility to provide the patient with individualized information (feedback) on their own mood patterns over time and their embedded context. The wealth of detailed individual information of daily life emotional experiences and their context may be beneficial to the individual patient in helping to gain more insight in his personal patterns of emotional experiences. Therefore, the next step in the treatment of depression is to examine whether ESM can be used as an intervention (ESM-I) to increase insights in personalized patterns of positive affect. Personalized feedback focused on positive affect and its context may help both the patient and the professional carer in their search for custom opportunities to increase the experience of positive affect, thus enabling recovery from depression. Therefore, chapter $\mathbf{5}$ is directed to examine the effectiveness and feasibility of ESM-derived feedback on daily life positive affect in the treatment of depression. 


\section{Aims and outline of the thesis}

The overall aim of this thesis was to explore the relevance of daily life emotional experiences as a source for a better understanding of mechanisms underlying the development of symptoms of a mental disorder and to explore whether a shift from macro-level clinical symptomatology to micro-level emotional experiences may have a place in treatment of mental disorders. The present thesis thus starts at the macrolevel of symptomatology (chapter 2) after which a shift to the micro-level of experiences (chapter 3, 4, and 5) was made.

The first objective was to examine the affective pathway to psychosis. In chapter $\mathbf{2}$ we looked in a general population sample at the macro-level of psychotic symptomatology and examined whether genetic liability for the development of a depressive disorder interacts with childhood adversity, leading to the development of psychotic symptoms. Furthermore, putative underlying affective mechanisms were examined. Using the same sample, in chapter $\mathbf{3}$, a shift was made from the macro-level of psychotic symptomatology to the micro-level of daily life emotional dynamics to examine the effect of 'the smallest building blocks' of daily life negative affective experiences on the development of paranoia and psychotic symptoms. In addition, it was examined whether the risk factors explored in chapter 2 impacted on this micro-level as well.

The second aim of this thesis was to examine whether a shift from the macro-level of symptomatology to the micro-level of daily life emotional experiences could contribute to clinical practice. Chapter $\mathbf{4}$ encompasses a literature review in which possibilities of the use of ESM in the diagnostic process and treatment of major depression were examined. In chapter 5, a randomized controlled trial was presented in which it was examined whether ESM-derived feedback on daily life positive affect would increase the effectiveness of pharmacological treatment of depression. Furthermore, it was examined whether ESM-I was considered feasible and useful by patients.

In chapter 6, a general discussion of the findings of this thesis is presented. Finally, chapter 7 closes with valorization, clinical implications and suggestions for future research. 


\section{References}

1. Bijl RV, Ravelli A, van Zessen G. Prevalence of psychiatric disorder in the general population: results of the Netherlands Mental Health Survey and Incidence Study (NEMESIS). Soc Psychiatry Psychiatr Epidemiol. 1998;33(12):587-95.

2. de Graaf R, Tuithof M, van Dorsselaer S, ten Have M. Comparing the effects on work performance of mental and physical disorders. Soc Psychiatry Psychiatr Epidemiol. 2012;47(11):1873-83.

3. American Psychiatric Association. Diagnostic and Statistical Manual of Mental Disorders (DSM-IV-TR). Washington, DC: American Psychiatric Association; 2000.

4. Angst J, Merikangas K. The depressive spectrum: diagnostic classification and course. J Affect Disord. 1997;45(1-2):31-40.

5. Cuijpers P, Smit F. Subthreshold depression as a risk indicator for major depressive disorder: a systematic review of prospective studies. Acta Psychiatr Scand. 2004;109(5):325-31.

6. World Health Organisation.The World Health Report 2001 - Mental health: new understanding, new hope. Geneva, Switzerland: World Health Organisation. 2001.

7. Rodriguez MR, Nuevo R, Chatterji S, Ayuso-Mateos JL. Definitions and factors associated with subthreshold depressive conditions: a systematic review. BMC Psychiatry. 2012;12(1):181.

8. Rapaport MH, Clary C, Fayyad R, Endicott J. Quality-of-life impairment in depressive and anxiety disorders. Am J Psychiatry. 2005;162(6):1171-8.

9. Judd LL, Paulus MP, Wells KB, Rapaport MH. Socioeconomic burden of subsyndromal depressive symptoms and major depression in a sample of the general population. Am J Psychiatry. 1996;153(11):1411-7.

10. Malla A, Payne J. First-episode psychosis: psychopathology, quality of life, and functional outcome. Schizophr Bull. 2005;31(3):650-71.

11. van Os J, Hanssen M, Bijl RV, Ravelli A. Strauss (1969) revisited: a psychosis continuum in the general population? Schizophr Res. 2000;45(1-2):11-20.

12. van Os J, Linscott RJ, Myin-Germeys I, Delespaul P, Krabbendam L. A systematic review and meta-analysis of the psychosis continuum: evidence for a psychosis proneness-persistence-impairment model of psychotic disorder. Psychol Med. 2009;39(2):179-95.

13. Johns LC, Cannon M, Singleton N, Murray RM, Farrell M, Brugha T, et al. Prevalence and correlates of selfreported psychotic symptoms in the British population. Br J Psychiatry. 2004;185(4):298-305.

14. Olfson M, Lewis-Fernandez R, Weissman MM, Feder A, Gameroff MJ, Pilowsky D, et al. Psychotic symptoms in an urban general medicine practice. Am J Psychiatry. 2002;159(8):1412-9.

15. Poulton R, Caspi A, Moffitt TE, Cannon M, Murray R, Harrington H. Children's self-reported psychotic symptoms and adult schizophreniform disorder: a 15-year longitudinal study. Arch Gen Psychiatry. 2000;57(11):1053-8.

16. van Nierop M, van Os J, Gunther N, Myin-Germeys I, de Graaf R, ten Have M, et al. Phenotypically continuous with clinical psychosis, discontinuous in need for care: evidence for an extended psychosis phenotype. Schizophr Bull. 2012;38(2):231-8.

17. Wigman JTW, van Nierop M, Vollebergh WAM, Lieb R, Beesdo-Baum K, Wittchen H-U, et al. Evidence that psychotic symptoms are prevalent in disorders of anxiety and depression, impacting on illness onset, risk, and severity-implications for diagnosis and ultra-high risk research. Schizophr Bull. 2012;38(2):247-57.

18. Krabbendam L, Myin-Germeys I, De Graaf R, Vollebergh W, Nolen WA, Iedema J, et al. Dimensions of depression, mania and psychosis in the general population. Psychol Med. 2004;34(7):1177-86.

19. Buckley PF, Miller BJ, Lehrer DS, Castle DJ. Psychiatric comorbidities and schizophrenia. Schizophr Bull. 2009;35(2):383-402.

20. Boks MPM, Leask S, Vermunt JK, Kahn RS. The structure of psychosis revisited: the role of mood symptoms. Schizophr Res. 2007;93(1-3):178-85.

21. Hanssen M, Peeters F, Krabbendam L, Radstake S, Verdoux H, van Os J. How psychotic are individuals with non-psychotic disorders? Soc Psychiatry Psychiatr Epidemiol. 2003;38(3):149-54.

22. van Rossum I, Lieb R, Wittchen H-U, van Os J. Affective dysregulation and reality distortion: a 10-year prospective study of their association and clinical relevance. Schizophr Bull. 2011;37(3):561-71. 
23. Varghese D, Scott J, Welham J, Bor W, Najman J, O'Callaghan M, et al. Psychotic-like experiences in major depression and anxiety disorders: a population-based survey in young adults. Schizophr Bull. 2011;37(2):389-93.

24. Maier W, Lichtermann D, Minges J, Hallmayer J, Heun R, Benkert O, et al. Continuity and discontinuity of affective disorders and schizophrenia. Results of a controlled family study. Arch Gen Psychiatry. 1993;50(11):871-83.

25. Argyropoulos SV, Landau S, Kalidindi S, Toulopoulou T, Castle DJ, Murray RM, et al. Twins discordant for schizophrenia: psychopathology of the non-schizophrenic co-twins. Acta Psychiatr Scand. 2008;118(3):214-9.

26. Mortensen PB, Pedersen MG, Pedersen CB. Psychiatric family history and schizophrenia risk in Denmark: which mental disorders are relevant? Psychol Med. 2010;40(2):201-10.

27. Wichers M, Schrijvers D, Geschwind N, Jacobs N, Myin-Germeys I, Thiery E, et al. Mechanisms of geneenvironment interactions in depression: evidence that genes potentiate multiple sources of adversity. Psychol Med. 2009;39(7):1077-86.

28. Read J, van Os J, Morrison AP, Ross CA. Childhood trauma, psychosis and schizophrenia: a literature review with theoretical and clinical implications. Acta Psychiatr Scand. 2005;112(5):330-50.

29. Varese F, Smeets F, Drukker M, Lieverse R, Lataster T, Viechtbauer W, et al. Childhood adversities increase the risk of psychosis: a meta-analysis of patient-control, prospective- and cross-sectional cohort studies. Schizophr Bull. 2012;38 (4):661-71.

30. Wichers M, Myin Germeys I, Jacobs N, Peeters F, Kenis G, Derom C, et al. Genetic risk of depression and stress-induced negative affect in daily life. Br J Psychiatry. 2007;191:218-23.

31. Wichers M, Geschwind N, Jacobs N, Kenis G, Peeters F, Derom C, et al. Transition from stress sensitivity to a depressive state: longitudinal twin study. Br J Psychiatry. 2009;195(6):498-503.

32. Myin Germeys I, van Os J, Schwartz JE, Stone AA, Delespaul PA. Emotional reactivity to daily life stress in psychosis. Arch Gen Psychiatry. 2001;58(12):1137-44.

33. Myin Germeys I, Delespaul P, van Os J. Behavioural sensitization to daily life stress in psychosis. Psychol Med. 2005;35(5):733-41.

34. Gilbert P. Evolution and depression: issues and implications. Psychol Med. 2006;36(03):287-97.

35. Selten J-P, Cantor-Graae E. Social defeat: risk factor for schizophrenia? Br J Psychiatry. 2005;187(2):101-2.

36. Caspi A, Sugden K, Moffitt TE, Taylor A, Craig IW, Harrington H, et al. Influence of life stress on depression: moderation by a polymorphism in the 5-HTT gene. Science. 2003;301(5631):386-9.

37. Bebbington P, Wilkins S, Jones P, Foerster A, Murray R, Toone B, et al. Life events and psychosis. Initial results from the Camberwell Collaborative Psychosis Study. Br J Psychiatry. 1993;162(1):72-9.

38. Kendler KS, Karkowski LM, Prescott CA. Causal relationship between stressful life events and the onset of major depression. Am J Psychiatry. 1999;156(6):837-41.

39. Myin-Germeys I, van Os J. Stress-reactivity in psychosis: evidence for an affective pathway to psychosis. Clin Psychol Rev. 2007;27(4):409-24.

40. de Vries MW. The experience of psychopathology: investigating mental disorders in their natural settings. Cambridge: Cambridge University Press; 1992.

41. Csikszentmihalyi M, Larson R. Validity and reliability of the Experience-Sampling Method. J Nerv Ment Dis. 1987;175(9):526-36.

42. Delespaul P. Assessing schizophrenia in daily life: the experience sampling method. Maastricht, the Netherlands: Maastricht University Press; 1995.

43. Simons CJP, Wichers M, Derom C, Thiery E, Myin-Germeys I, Krabbendam L, et al. Subtle gene-environment interactions driving paranoia in daily life. Genes Brain Behav. 2009;8(1):5-12.

44. Myin-Germeys I, Oorschot M, Collip D, Lataster J, Delespaul P, van Os J. Experience sampling research in psychopathology: opening the black box of daily life. Psychol Med. 2009;39:1533-47.

45. Trull TJ, Ebner-Priemer U. Ambulatory assessment. Annu Rev Clin Psychol. 2013;9(1):151-76.

46. Wichers M, Peeters F, Geschwind N, Jacobs N, Simons CJP, Derom C, et al. Unveiling patterns of affective responses in daily life may improve outcome prediction in depression: a momentary assessment study. J Affect Disord. 2010;124(1-2):191-5. 
47. Thewissen V, Bentall RP, Oorschot $\mathrm{M}$, à Campo J, van Lierop $\mathrm{T}$, van $\mathrm{Os} \mathrm{J}$, et al. Emotions, self-esteem, and paranoid episodes: an experience sampling study. Br J Clin Psychol. 2011;50(2):178-95.

48. Heron KE, Smyth JM. Ecological momentary interventions: incorporating mobile technology into psychosocial and health behaviour treatments. Br J Health Psychol. 2010;15(1):1-39.

49. Geschwind N, Nicolson NA, Peeters F, van Os J, Barge-Schaapveld D, Wichers M. Early improvement in positive rather than negative emotion predicts remission from depression after pharmacotherapy. Eur Neuropsychopharmacol. 2011;21(3):241-7.

50. Wichers MC, Barge-Schaapveld DQCM, Nicolson NA, Peeters F, de Vries M, Mengelers R, et al. Reduced stress-sensitivity or increased reward experience: the psychological mechanism of response to antidepressant medication. Neuropsychopharmacology. 2009;34(4):923-31.

51. Parati G, Omboni S, Bilo G. Why is out-of-office blood pressure measurement needed? Home blood pressure measurements will increasingly replace ambulatory blood pressure monitoring in the diagnosis and management of hypertension. Hypertension. 2009;54(2):181-7.

52. Nardacci EA, Bode BW, Hirsch IB. Individualizing care for the many: the evolving role of professional continuous glucose monitoring systems in clinical practice. Diabetes Educ. 2010;36(Suppl 1):4S-19S.

53. Keijsers NILW, Horstink MWIM, Gielen SCAM. Ambulatory motor assessment in Parkinson's disease. Mov Disord. 2006;21(1):34-44.

54. Koster EHW, De Raedt R, Leyman L, De Lissnyder E. Mood-congruent attention and memory bias in dysphoria: exploring the coherence among information-processing biases. Behav Res Ther. 2010;48(3):219-25.

55. Denicoff K, Leverich G, Nolen W, Rush A, McElroy S, Keck P, et al. Validation of the prospective NIMHLife-Chart Method (NIMH-LCMTM-p) for longitudinal assessment of bipolar illness. Psychol Med. 2000;30(06):1391-7.

56. Denicoff KD, Ali SO, Sollinger AB, Smith-Jackson EE, Leverich GS, Post RM. Utility of the daily prospective National Institute of Mental Health Life-Chart Method (NIMH-LCM-p) ratings in clinical trials of bipolar disorder. Depress Anxiety. 2002;15(1):1-9.

57. Wichers M, Hartmann JA, Kramer IMA, Lothmann C, Peeters F, van Bemmel L, et al. Translating assessments of the film of daily life into person-tailored feedback interventions in depression. Acta Psychiatr Scand. 2011;123(5):402-3.

58. Wichers M, Simons CJP, Kramer IMA, Hartmann JA, Lothmann C, Myin-Germeys I, et al. Momentary assessment technology as a tool to help patients with depression help themselves. Acta Psychiatr Scand. 2011;124(4):262-72.

59. Trull TJ, Ebner-Priemer UW. Using experience sampling methods/ecological momentary assessment (ESM/EMA) in clinical assessment and clinical research: introduction to the special section. Psychol Assess. 2009;21(4):457-62.

60. World Health Organization. Depression. A global public health concern. Geneva, Switzerland: World Health Organization. 2012.

61. Wiles N, Thomas L, Abel A, Ridgway N, Turner N, Campbell J, et al. Cognitive behavioural therapy as an adjunct to pharmacotherapy for primary care based patients with treatment resistant depression: results of the CoBalT randomised controlled trial. Lancet. 2013;381(9864):375-84.

62. Trivedi MH, Rush AJ, Wisniewski SR, Nierenberg AA, Warden D, Ritz L, et al. Evaluation of outcomes with citalopram for depression using measurement-based care in STAR* D: implications for clinical practice. Am J Psychiatry. 2006;163(1):28-40.

63. Dimidjian S, Hollon SD, Dobson KS, Schmaling KB, Kohlenberg RJ, Addis ME, et al. Randomized trial of behavioral activation, cognitive therapy, and antidepressant medication in the acute treatment of adults with major depression. J Consult Clin Psychol. 2006;74(4):658-70.

64. Fuchs CZ, Rehm LP. A self-control behavior therapy program for depression. J Consult Clin Psychol. 1977;45(2):206-15.

65. Lewinsohn PM. A behavioral approach to depression. In: RJ Friedman \& Katz M, editor. The psychology of depression: contemporary theory and research. Oxford, England: Wiley; 1974. p. 157-78. 



\section{PART 1}

\section{Experience Sampling Method}

for understanding psychopathology 


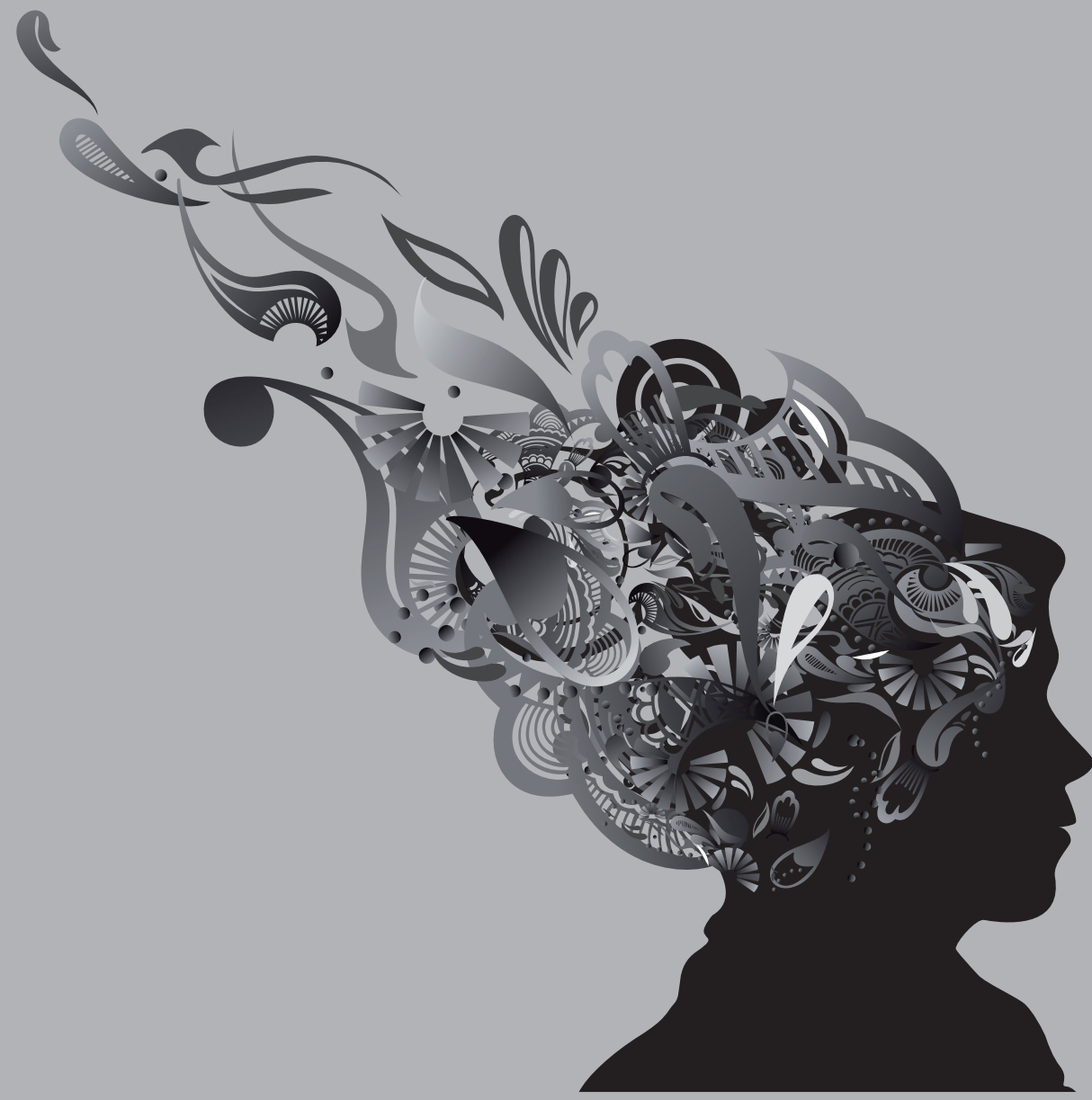




\section{CHAPTER 2}

\section{Evidence that genes for depression impact on the pathway from trauma}

to psychotic-like symptoms by occasioning emotional dysregulation

IMA. Kramer ${ }^{1,2}$, CJP. Simons ${ }^{1,2}$, I. Myin-Germeys ${ }^{2}$, N. Jacobs ${ }^{2,3}$, C. Derom ${ }^{4}$, E. Thiery ${ }^{5}$, J. van Os ${ }^{2,6}$, M. Wichers ${ }^{2}$

${ }^{1}$ GGzE, Institute of Mental Health Care Eindhoven and the Kempen, Eindhoven, the Netherlands

${ }^{2}$ Department of Psychiatry and Psychology, South Limburg Mental Health Research and Teaching Network, EURON, Maastricht University, the Netherlands ${ }^{3}$ Faculty of Psychology, Open University of the Netherlands, Heerlen, the Netherlands

${ }^{4}$ Department of Human Genetics, University Hospital Gasthuisberg, Katholieke Universiteit Leuven, Leuven, Belgium

${ }^{5}$ Association for Scientific Research in Multiple Births, Ghent, Belgium

${ }^{6}$ King's College London, Department of Psychosis Studies, Institute of Psychiatry, London, UK 


\section{Abstract}

Background: Genes for depression may act by making individuals more sensitive to childhood trauma. Given that childhood adversity is a risk factor for adult psychosis and symptoms of depression and psychosis tend to cluster within individuals and families, the aim was to examine whether the association between childhood adversity and psychotic-like symptoms is moderated by genetic liability for depression. A secondary aim was to determine to what degree a depression-related increase in stress-sensitivity or depressive symptoms themselves occasioned the moderating effect.

Methods: Female twins $(n=508)$ completed both prospective and retrospective questionnaires regarding childhood adversity, Symptom Check List-90-Revised (SCL90-R), SCID-I (psychotic symptoms) and psychotic trait liability (the Community Assessment of Psychic Experiences (CAPE)). Stress sensitivity was indexed by appraisals of event-related stress and negative affect (NA) in the flow of daily life, assessed with momentary assessment technology for five consecutive days. Multilevel regression analyses were used to examine moderation of childhood adversity by genetic liability for depression in the prediction of follow-up psychotic symptoms.

Results: The effect of childhood adversity was significantly moderated by genetic vulnerability for depression in the model of both follow-up psychotic symptoms (SCL90-R) and follow-up psychotic trait liability (CAPE). The moderation by genetic liability was mediated by depressive experience, but not by stress sensitivity.

Conclusions: Genetic liability for depression may potentiate the pathway from childhood adversity to psychotic-like symptoms through dysfunctional emotional processing of anomalous experiences associated with childhood trauma. 


\section{Introduction}

Currentevidence suggests that childhood adversity is associated not only with depression (1-3), but also with psychotic outcomes. Although reviews on the association between childhood trauma and psychosis are not consistent (4-6), numerous methodologically robust studies, including several prospective studies (7-12), have all demonstrated associations between childhood trauma and psychotic symptoms/psychotic disorder (13-20). The pattern of shared environmental risk indexed by childhood trauma is of major interest because depression and psychosis are found to cluster in the same individuals (21-24) and also in the same families $(25,26)$, suggesting shared genetic liability.

There is strong evidence that genetic risk for depression is expressed in part as sensitivity to childhood trauma (27-29). Given that major depression and psychotic disorder share genetic risk, it is attractive to hypothesize that (i) genetic liability for depression moderates the effect of an environmental risk factor, such as childhood adversity, on the development of psychotic outcomes and (ii) moderation is occasioned by affective mechanisms (Figure 1). Daily life stress sensitivity has been identified as an intermediate phenotype for both psychosis and depression; that is, it is associated with genetic risk for these disorders (30-33). In addition, childhood adversity, alone and in interaction with genetic liability for depression, has been shown to increase adult daily life stress sensitivity $(29,34)$. Stress sensitivity has also been shown to predict follow-up depressive and psychotic symptoms. Therefore, genetic liability for depression may act on risk for both psychosis and depression by increasing daily life stress sensitivity (see Figure 1., hypothesis 1). However, another possibility is that genetic risk on the pathway to vulnerability is shared until the emergence of depressive symptoms themselves (see Figure 1., hypothesis 2), which may impact on the subsequent emergence of psychosis $(35,36)$. Depressive symptomatology may induce misassignment of emotional salience, which can give rise to the onset of psychotic symptoms (24).

In the current study we examined whether genetic liability for depression moderates the effect of childhood adversity on the development of follow-up psychotic symptoms. We hypothesized that stress sensitivity or experience of depressive symptoms may represent potential mechanisms underlying this interaction (Figure 1), that is, the interaction between genetic liability for depression and childhood adversity is mediated by stress sensitivity or depressive symptomatology. Analyses were conducted in a large general population twin sample, making it possible to build interactive models with genetic liability for depression as hypothesized. It has been shown that psychotic experiences exist as a continuous distribution of symptoms in the general population (37), ranging from subclinical psychotic experiences to a psychotic disorder. The 
median prevalence of these psychotic experiences in the general population is $5.3 \%$, with a median incidence of $3.1 \%$ (38). Subclinical psychosis may be more sensitive to detect associations with risk factors in general population studies. The advantage of a general population design is that effects of illness characteristics, such as medication or severe psychopathology, on the variables under examination can be avoided.

Hypothesis 1: The interaction between depression genes and childhood adversity results in an increased stress sensitivity which is the common causal basis for both depression and psychosis

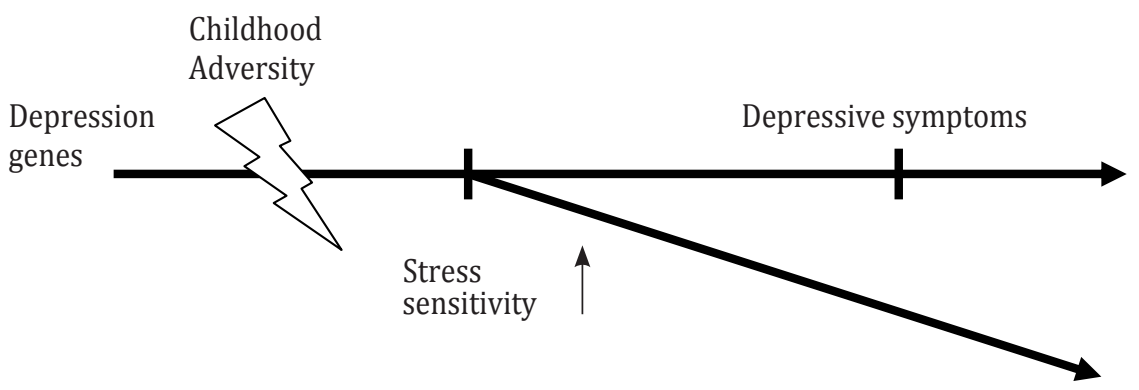

Psychotic

symptoms

Hypothesis 2: The interaction between depression genes and childhood adversity results in emotional dysregulation which causes psychosis

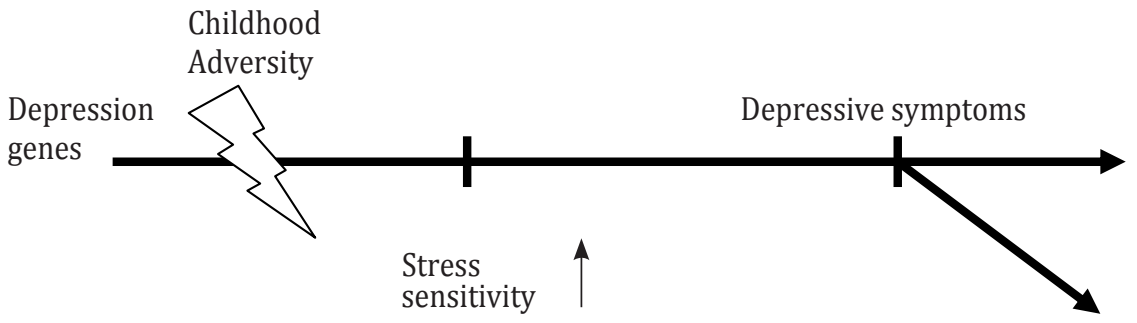

Psychotic symptoms

Figure 1 | Model of the hypothesized moderating influence of the genetic disposition for depression on the relationship between childhood trauma and the development of psychosis. Stress sensitivity and experience of depression are hypothesized to represent underlying mechanisms. 


\section{Method}

\section{Sample}

The study sample consisted of 621 general population female twins and their non-twin sisters ( $n=44)$, aged 18-61 years, from Flanders, Belgium. Given the evidence for gender differences in the type of environmental stressors that are associated with psychosis (39) and depression (40), a female-only sample was chosen to improve homogeneity. The intended age range was young to middle adulthood (mean age at baseline 27.9 years, $S D=7.9)$. The sample was relatively highly educated $(63 \%$ college or university degree, $36 \%$ secondary education, and 1\% primary education only). The twins were recruited from the East Flanders Prospective Twin Survey (EFPTS) (218 pairs) and from birth registers of Flemish municipalities in Belgium. The EFPTS population-based survey has prospectively recorded all multiple births in the province of East Flanders since 1964 (41, 42). Zygosity was determined through sequential analysis based on sex, fetal membranes, blood groups, and DNA fingerprints. The project was approved by the local ethics committee and all participants gave written informed consent.

\section{Procedure}

The sample was assessed at five time points, including a baseline (T0) and four followup measurements (T1-T4). The average number of days between T0 and T1 was 132, 91 between $\mathrm{T} 1$ and T2, 116 between T2 and T3, and 91 between T3 and T4. The first interview was at individuals' homes, and follow-up data were collected using questionnaires and telephone interviews. All interviews were performed by trained research psychologists or graduate psychological assistants. The Experience Sampling Method (ESM) was only carried out at T0.

\section{Measurements}

\section{Childhood adversity}

Childhood adversity was measured at baseline (T0) using the shortened version of the 70-item Childhood Trauma Questionnaire (CTQ) (43-45). The shortened version (46) comprises 25 items that assess five dimensions of childhood maltreatment: (1) physical abuse, (2) emotional abuse, (3) sexual abuse, (4) physical neglect, and (5) emotional neglect. At the request of the Flemish Twin Register, the four most explicit items concerning sexual and physical abuse were omitted, resulting in a 21 -item self-report questionnaire (e.g. 'I was maltreated', 'I was beaten often', 'I was abused', 'There was not enough food', and 'I was neglected'). Items were scored on a scale of 1 (never true) to 5 (very often true). Cronbach's $\alpha$ for this 21 -item questionnaire was 0.93 . 


\section{Depressive symptoms}

The Structured Clinical Interview for DSM-IV Axis I disorders (SCID-I) (47) was administered by trained psychologists to obtain current and lifetime diagnosis of major depressive disorder of both proband and co-twin. The SCID was administered twice, at baseline (T0) and at follow-up period 4 (T4).

A continuous measure of depressive symptoms was obtained using the Symptom Check List-90-Revised (SCL-90-R) $(48,49)$, which is a widely used 90 -item self-report questionnaire measuring general psychological distress. The depression subscale consists of 13 items. The SCL-90-R was administered five times, including baseline (T0) and all four follow-up periods (T1-4).

\section{Psychotic symptoms}

Psychotic symptoms were measured in three ways. First, SCID psychotic symptoms were assessed with the SCID-I (47). The sum score of the subscales delusions (consisting of 15 items) and hallucinations (consisting of eight items) was used to rate SCID psychotic symptoms. The SCID was administered twice, at T0 and at T4 follow-up.

Second, psychotic symptoms were assessed with the SCL-90-R $(48,49)$. The mean score of the subscales paranoid ideation (consisting of six items) and psychoticism (consisting of 10 items) was used to rate SCL-90-R psychotic symptoms. The SCL-90-R was administered five times, that is at all T0-T4 measurement occasions.

Third, the measurement of psychotic trait liability consisted of the Community Assessment of Psychic Experiences (CAPE) $(22,50,51)$, which is based on the Peters et al. Delusions Inventory (PDI) (52), modified to also include hallucinatory experiences. The CAPE is a 42-item self-report questionnaire to assess lifetime psychotic experiences. It measures positive (20 items), negative (14 items) and depressive (eight items) dimensions of the subclinical psychosis phenotype in the general population. A new variable was created consisting of the mean score of the positive symptom dimension of the CAPE. The CAPE was administered three times, at T0 and at T2 and T4.

\section{Stress sensitivity}

Stress sensitivity was measured in daily life as appraisals of minor daily events and their effect on negative affect (NA), collected within the ESM framework. ESM is a momentary assessment technique to assess subjects in their daily living environment and has been extensively validated for the use of immediate effects of stressors on mood (30,53-58). Subjects received a digital wristwatch and a set of ESM self-assessment forms collected in a booklet for each day. The wristwatch was programmed to emit a signal (beep) at an unpredictable moment in each of ten 90 -minute time blocks between 730 and $2230 \mathrm{~h}$, on five consecutive days. Compliance to the ESM protocol was evaluated in a random 
subsample (58 subjects; 1938 observations) of the sample used in the current study and was very high (96.4\%) (59).

Measures of daily life stress and NA were collected at each beep within the ESM framework. To measure ESM event-related stress, subjects were asked to report the most important event that happened between the current and the previous beep. This event was subsequently rated on a seven-point bipolar scale (from -3 = very unpleasant, $0=$ neutral, to 3 = very pleasant). The scale was reversed so that higher scores represent higher disliking the event (event stress). NA was assessed at each beep with six mood adjectives ('insecure', 'lonely', 'anxious', 'low', 'guilty' and 'suspicious') rated on a sevenpoint Likert scale. NA was calculated by averaging the NA items weighted for the rotated factor loadings of these items obtained in principal component analysis (Cronbach's $\alpha=$ .76). Stress sensitivity was defined as NA reactivity towards small daily life stressors in the flow of an individual's daily life. To create the variable stress sensitivity, the weighted mean NA was regressed on ESM event-related stress.

\section{Statistical analysis}

First, the effect of childhood adversity on the development of psychotic symptoms over the follow-up period was examined. Second, a new variable was constructed that represented the individual's genetic vulnerability to depression. This variable was coded 0 in case of no genetic vulnerability (a twin sister without a lifetime depression diagnosis), 1 for having a dizygotic (DZ) sister with a lifetime depression diagnosis and 2 for a monozygotic (MZ) sister with a lifetime depression diagnosis $(29,60)$. The higher an individual loads on this factor, the greater the genetic liability for major depression. To examine whether the effect of childhood adversity on follow-up psychotic outcomes was moderated by genetic vulnerability to depression, a multilevel linear regression model was used to account for clustering of multiple measurements of psychosis (level 1) within subjects (level 2) and clustering of subjects within twin pairs (level 3). Follow-up psychotic outcomes, adjusted for their baseline values, were modelled at three separate levels of (i) psychotic symptoms, indexed by the SCL-90-R at T1-T4, (ii) SCID psychotic symptoms, indexed by the SCID at T4, and (iii) trait liability indexed by the CAPE positive symptom dimension at T2 and T4. Effect sizes on psychotic outcomes at different levels of genetic liability and childhood adversity were calculated from the model containing the interaction, forming the appropriate linear combinations with the STATA LINCOM routine (61). Main effects and interactions were assessed by Wald tests (62).

Finally, in case of significant interaction, it was examined whether stress sensitivity or depressive symptoms mediated the interaction effects on follow-up psychotic outcomes. For this purpose, the interaction model was additionally controlled for 
stress sensitivity or mean depressive symptoms on either the SCL-90-R or the CAPE respectively. Furthermore, follow-up psychotic outcomes were regressed on these putative mediators to demonstrate that variations in these variables significantly accounted for variations in the outcome variables, thus meeting all mediator conditions. Data were analysed with the XTREG and XTMIXED modules in STATA v. 11.0 (61) .

All models of psychotic outcomes were adjusted for their baseline value. By correcting for the baseline psychosis value, measures of follow-up psychotic outcomes thus indexed increase in psychotic symptomatology over the follow-up period. All variables included in the analyses were standardized (by dividing the variables by their between-subject standard deviation), yielding standardized effect sizes ( $\beta$ ). This allows for a comparison of effect sizes across different measurements.

\section{Results}

\section{Subjects' characteristics}

Of the total sample of 621 subjects, all 44 non-twin sisters were excluded. One subject was excluded because of incorrect data on zygosity. Another 68 subjects were excluded because of missing data on either childhood adversity or co-twin lifetime depression diagnosis. This resulted in a dataset of 508 subjects (312 MZ and 196 DZ subjects), with a mean age of 27.1 years $(S D=7.4$, range 18-46). The majority of the subjects were currently employed (59.0\% employed, $38.3 \%$ students, $2.4 \%$ unemployed, and $0.4 \%$ on sick leave). A small number of subjects used psychotropic medication: 21 (4.1\%) used antidepressants, one $(0.2 \%)$ antipsychotics, four $(0.8 \%)$ anxiolytics, and eight $(1.6 \%)$ hypnotics.

For all three psychosis variables, subjects were included in the analyses if at least one of the follow-up measurements was completed. Mean follow-up psychotic symptoms score assessed by the SCL-90-R ranged from 1 to 3.1 (median of 1.2), the sum score of follow-up psychotic symptoms assessed by the SCID ranged from 0 to 4 (median of 0 ), and mean follow-up psychotic liability measured by the CAPE ranged from 1 to 1.9 (median of 1.1). Owing to partial missing data on the follow-up measurements of psychotic experiences, differences in group size exist, ranging from 409 subjects for SCID measurements to 476 for SCL-90-R scores and 463 subjects for CAPE measurements. No differences could be demonstrated between subjects who dropped out after baseline compared to subjects who did not for SCL-90-R, SCID or CAPE psychotic symptoms.

The average childhood adversity score was $1.7(S D=.6)$. Sixty-one subjects $(12.0 \%)$ had one or more SCID psychotic symptoms at baseline. Twenty-three subjects (4.5\%) had a current depression at baseline. Eighty-seven subjects $(17.2 \%)$ had a lifetime depression diagnosis. These prevalence rates are comparable to the 1-month prevalence of $3.4 \%$ 
and the life-time prevalence of $20.4 \%$ reported in another female general population (63). Ninety probands (17.7\%) had a co-twin with a lifetime diagnosis of depression. Eleven percent of the mothers of the twins were diagnosed with depression, $0.6 \%$ with bipolar disorder, and $0.2 \%$ with a psychotic disorder. With respect to the fathers of the twins: $5.8 \%$ were diagnosed with depression, $0.8 \%$ with bipolar disorder, and $0 \%$ with a psychotic disorder. Independence of the variables of the interaction term (adversity and genetic liability for depression) was demonstrated $(\beta=.05, p=.68$ for the DZ twins and $\beta=-.04, p=.75$ for the MZ twins).

\section{Association between childhood adversity and follow-up psychotic outcomes}

Childhood adversity was associated with the development of follow-up SCL-90-R psychotic symptoms $(\beta=.16, p \leq .001)$ and with follow-up CAPE psychotic liability $(\beta=.13, p \leq .001)$. A trend towards significance was demonstrated between childhood adversity and the development of follow-up SCID psychotic symptoms $(\beta=.09, p=.08)$.

\section{Moderation by genetic liability for depression}

Genetic liability for depression significantly moderated the effect of childhood adversity on follow-up SCL-90-R psychotic symptoms. High genetic risk was associated with a greater effect of childhood adversity on SCL-90-R psychotic symptoms. MZ co-twin lifetime depression, compared to no co-twin depression (the reference category), significantly increased the effect of childhood adversity on follow-up SCL-90-R psychotic symptoms, whereas the effect of DZ co-twin lifetime depression was not significantly different compared to the effect of no co-twin depression (Table 1 and Figure 2a).

Similarly, the effect of childhood adversity on follow-up CAPE trait liability was greater for subjects with a high genetic risk for depression. MZ co-twin lifetime depression, compared to no co-twin depression, significantly increased the effect of childhood adversity on follow-up CAPE trait liability, whereas the effect of DZ co-twin lifetime depression was comparable to the effect of no co-twin depression (Table 1 and Figure 2b).

The interaction effect on follow-up SCID psychotic symptoms was not significant.

\section{Stress sensitivity as a mediator of interaction}

To examine whether stress sensitivity might represent the mechanism underlying the significant interaction effect between genetic liability for depression and childhood adversity in the models of follow-up SCL-90-R psychotic symptoms and follow-up CAPE trait liability, analyses were carried out again controlling for stress sensitivity. This did not result in a large or significant reduction of the interaction effect between genetic liability for depression and childhood adversity in the model of SCL-90-R or CAPE 
Table 1 | Overview of the interaction models with $\beta$ coefficient, $\chi^{2}, p$ value, and number of subjects and twin pairs for each separate analysis on three different scales of psychotic-like symptoms (SCL-90-R, SCID, and CAPE).

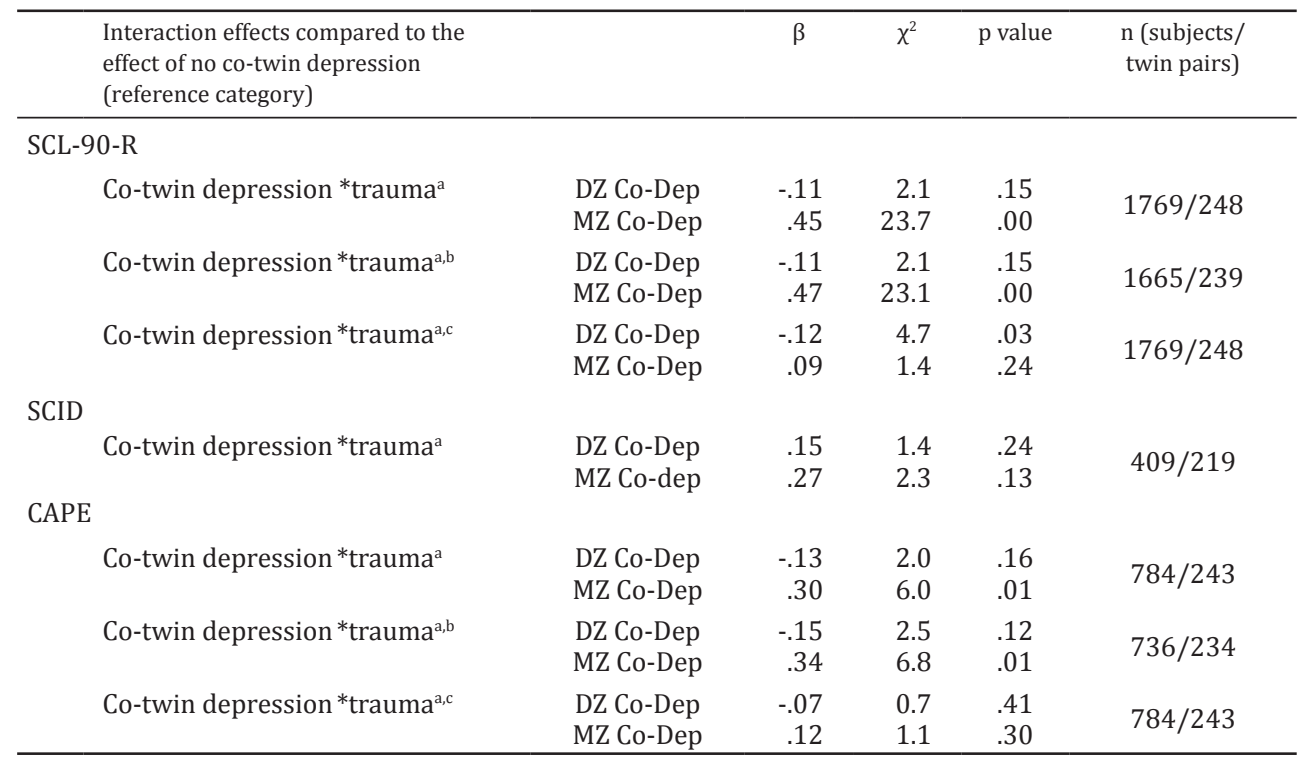

SCL-90-R, Symptom Checklist-90-Revised; SCID, Structured Clinical Interview for DSM-IV Axis I disorders; CAPE, Community Assessment of Psychic Experiences; MZ, monozygotic; DZ, dizygotic; Co-Dep, co-twin lifetime depression.

a Analyses were corrected for current psychotic symptoms

b Analyses that demonstrated a significant interaction effect were corrected for stress sensitivity.

c Analyses that demonstrated a significant interaction effect were corrected for mean depressive symptoms.

A

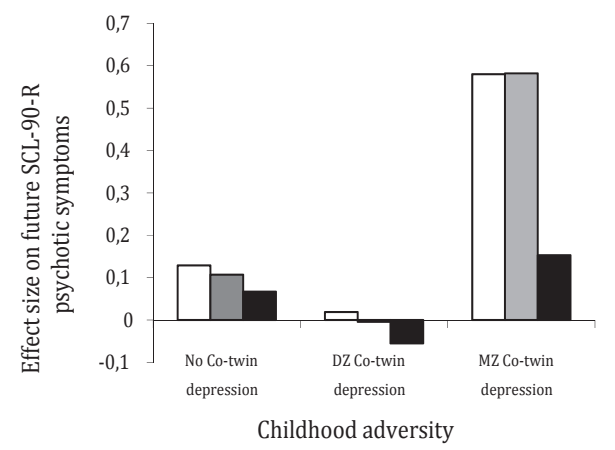

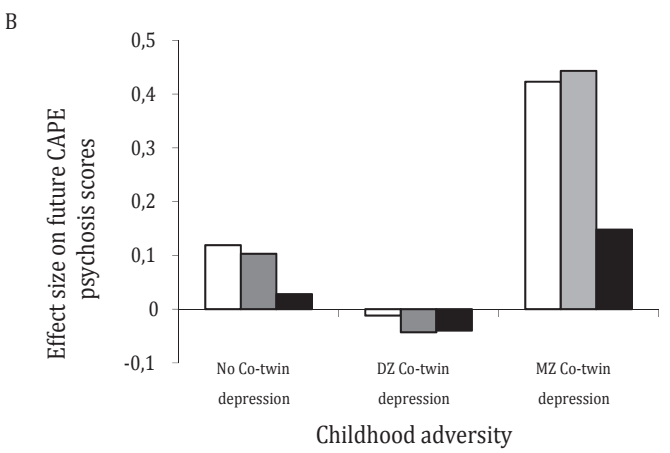

Figure 2 | Standardized effect sizes of childhood adversity on (A) follow-up SCL-90-R psychotic symptoms and (B) follow-up CAPE psychosis scores with and without correction for possible underlying mechanisms, stratified by level of genetic vulnerability to depression

Interaction with childhood adversity; $\square$, interaction corrected for stress sensitivity;

口, interaction corrected for emotional dysregulation. 
psychotic outcomes. If anything, controlling for stress sensitivity resulted in an increase in effect size $(0.3 \%$ and $4.7 \%$ increase for SCL-90-R and CAPE respectively). Having an MZ co-twin with lifetime depression, compared to a co-twin without depression, still moderated the effect of childhood adversity in the model of both follow-up SCL-90-R psychotic symptoms (Table 1 and Figure 2a) and follow-up CAPE trait liability (Table 1 and Figure 2b).

\section{Depressive symptoms as a mediator of the interaction}

Similarly, to examine whether depressive experiences might represent the mechanism underlying the significant interaction effect between genetic liability for depression and childhood adversity in the models of follow-up SCL-90-R psychotic symptoms and follow-up CAPE trait liability, analyses were carried out again controlling for mean SCL90-R or mean CAPE depressive symptoms. This resulted in a loss of significance of the interaction effect between genetic liability for depression and childhood adversity on both the follow-up SCL-90-R and follow-up CAPE scores (a 73.6\% and 64.9\% decrease in effect size respectively). Having an MZ co-twin with a lifetime depression, compared to a co-twin without depression, no longer moderated the effect of childhood adversity in the model of both follow-up SCL-90-R psychotic symptoms (Table 1 and Figure 2a), and follow-up CAPE trait liability (Table 1 and Figure 2b). Sensitivity analyses that excluded depressive items that overlap with negative symptoms, such as vital symptoms and anhedonia, yielded similar results. In addition, the results showed that depressive symptoms preceded the development of psychotic-like symptoms because mean baseline depressive symptoms on either the SCL-90-R or the CAPE predicted follow-up psychotic-like symptoms ( $\beta=.23, p \leq .001$ and $\beta=.16, p \leq .001$ respectively).

\section{Discussion}

In this study we aimed to investigate whether genetic liability for depression acts as a moderator in the association between childhood adversity and the development of psychotic-like symptoms.

First, we demonstrated that, in general population females, genetic liability for depression moderates the association between childhood adversity and follow-up psychotic-like symptoms. In females, a high genetic risk for depression increased the effect of childhood adversity on developing psychotic-like symptomatology over the follow-up period. This finding was replicated in two separate measures of psychotic-like symptoms. The identification of genetic liability for depression as a moderator of the effect of childhood adversity on the development of follow-up psychotic-like outcomes, may suggest a partially shared genetic pathway to both depression and psychosis in 
females (64).

Second, two hypothesized mechanisms underlying the interaction effect in the development of follow-up psychotic-like symptoms were tested, stress sensitivity and emotional dysregulation. The upper figure in Figure 1 depicts hypothesis 1, where increased stress sensitivity resulting from childhood adversity $\mathrm{x}$ genetic liability to depression is the hypothesized shared pathway to psychotic-like symptoms. Although stress sensitivity has been identified previously as an underlying intermediate phenotype for both depression and psychosis (30-32), the current results support the second hypothesis in which increased risk for psychotic-like symptoms emerges from the experience of depressive symptoms rather than from increased stress sensitivity (Figure 1, hypothesis 2). This indicates that, in females, the occurrence of depressive symptoms plays a role in the development of psychotic-like symptoms. The finding is in accordance with other studies that showed that depressive symptoms precede the onset of psychotic disorders (65-67). Because stress sensitivity is a risk factor for future depressive symptoms $(29,30,68)$, stress sensitivity is probably on the pathway to psychosis indirectly via a path where stress sensitivity increases the experience of depressive symptoms, which in turn induce psychotic-like symptoms. The fact that depressive symptoms may mediate the onset of psychotic-like symptoms is of relevance to the development of new effective interventions and prevention strategies to battle psychosis. Furthermore, the partially shared etiology reported in the current study [gene-environment interaction (GxE) on risk for depressive symptoms] provides an explanation for the observed co-occurrence of the two symptom clusters in psychotic patients.

Emotional processes are involved in attributing salience to experiences and are therefore undeniably involved in the development of psychotic symptoms $(66,69-75)$. According to the cognitive model of psychosis of Garety and colleagues $(76,77)$, stress triggers emotional and cognitive changes that can, in a biopsychosocial vulnerable individual, result in appraisals of the origins of anomalous experiences as externally caused. Externalizing appraisal, the judgment that the experience is externally caused, can lead to positive symptoms of psychosis, for example delusions. Owing to biased appraisals, emotional processes can also lead to misassignment of emotional salience to experiences. This mechanism has recently been proposed as an intermediate (cognitive) mechanism that facilitates the transition from emotional dysregulation to reality distortion (24) . Another mechanism leading to psychosis may be the tendency to worry, which is associated with both the onset and persistence of hallucinations (70). For example, the experience of auditory hallucinations has been related to higher scores on metacognitive beliefs concerning both positive beliefs about worry and negative beliefs about uncontrollability and danger (78). Anomalous experiences can lead, by 
means of delusional interpretation of these experiences, to an increased tendency to worry $(79,80)$ and a depressive response $(69,80,81)$. Specific aspects of the delusional interpretation have been related to emotional distress and depression (82). The results also emphasize the likelihood of shared neurobiological mechanisms involved in psychosis and depression. Shared mechanisms may involve altered hypothalamicpituitary-adrenal (HPA) axis activation (83-85) and neurobiological sensitization mechanisms $(29,86)$. Prevention of psychosis could take place not only at the stage of the emergence of depressive symptoms, but also at the stage where certain individuals, following experience of depressive symptoms, make the transition to psychosis. Therefore, efforts should be put into elucidating the underlying mechanism of this transition and understanding how emotional, cognitive and biological factors may act together in the transition from depressive to psychotic symptoms.

\section{Psychosis: a dimensional phenotype}

No significant interaction effects on follow-up SCID scores could be demonstrated, probably because of a lack of power. Psychotic symptoms as measured using the SCID are rare in the general population and were experienced only by 38 subjects $(9.3 \%)$ in this sample at the follow-up measurement. However, evidence has accumulated that the phenotype of psychosis, like other psychopathological phenotypes, is dimensional in nature $(38,50,65,87-93)$ and that the much more frequent subclinical psychotic experiences share a similar etiological background with the more severe SCID psychotic symptoms $(37,38,50,94)$. Therefore, examining subclinical psychotic symptoms in the general population is i) informative with regard to the clinical expression of these symptoms, ii) has much higher power than when examining clinical symptoms or diagnosis of psychosis, and iii) does not suffer from confounding due to medication or other disease status-associated factors, which is often the case in clinical studies. The current results showed two similar and significant interaction effects for the two separate measures of subclinical psychotic symptoms used in this study. The fact that the interaction effect between genetic liability and early adversity was replicated in another measure of psychotic-like symptoms supports the results.

\section{Limitations}

There were several methodological issues. Childhood adversity was measured with a self-report questionnaire and retrospectively. The measure for childhood adversity, the short version of the CTQ, was adapted on request of the Flemish Twin Register. Although the total average childhood adversity score may be somewhat lower because of the omission of the four most explicit items, the interaction between childhood adversity and genetic liability for depression was significant. Omission of these items may have 
resulted in reduced effect sizes of early adversity on psychosis. Thus, the current results probably reflect a conservative estimation of the effects in the general population. The low average childhood adversity score and its limited variability may also indicate that the subjects sample is a privileged and relatively healthy group.

Subjects were female with a high mean educational level. Because of gender differences in risk factors for different pathways to psychosis (33), the results of the study may not be generalized to men and to those with lower educational level.

A limitation of ESM in the measurement of stress sensitivity is that the effects between stress appraisals and NA can go in either direction. However, either explanation bears clinical relevance.

\section{Acknowledgements}

This research was supported by the Netherlands Organisation for Scientific Research; the Fund for Scientific Research, Flanders and Twins, a non-profit association for scientific research in multiple births (Belgium) (to the East Flanders Prospective Survey). All twins are thanked for their cooperation. Dr. M.C. Wichers was supported by the Dutch Medical Council (VENI grant nr. 916.76.147). Dr. I. Myin-Germeys was supported by a 006NARSAD Young Investigator award and by the Dutch Medical Council (VENI and VIDI Grant).

\section{Declaration of interest:}

None. 


\section{References}

1. Chapman DP, Whitfield CL, Felitti VJ, Dube SR, Edwards VJ, Anda RF. Adverse childhood experiences and the risk of depressive disorders in adulthood. J Affect Disord. 2004;82(2):217-25.

2. Heim C, Nemeroff CB. The role of childhood trauma in the neurobiology of mood and anxiety disorders: preclinical and clinical studies. Biol Psychiatry. 2001;49(12):1023-39.

3. Wiersma JE, Hovens JGFM, van Oppen P, Giltay EJ, van Schaik DJF, Beekman ATF, et al. The importance of childhood trauma and childhood life events for chronicity of depression in adults. J Clin Psychiatry. 2009;70(7):983-9.

4. Read J, van Os J, Morrison AP, Ross CA. Childhood trauma, psychosis and schizophrenia: a literature review with theoretical and clinical implications. Acta Psychiatr Scand. 2005;112(5):330-50.

5. Larkin W, Read J. Childhood trauma and psychosis: evidence, pathways, and implications. J Postgrad Med. 2008;54(4):287-93.

6. Bendall S, Jackson HJ, Hulbert CA, McGorry PD. Childhood trauma and psychotic disorders: a systematic, critical review of the evidence. Schizophr Bull. 2008;34(3):568-79.

7. Deloore E, Drukker M, Gunther N, Feron F, Deboutte D, Sabbe B, et al. Childhood negative experiences and subclinical psychosis in adolescence: a longitudinal general population study. Early Interv Psychiatry. 2007;1:201-7.

8. Schreier A, Wolke D, Thomas K, Horwood J, Hollis C, Gunnell D, et al. Prospective study of peer victimization in childhood and psychotic symptoms in a nonclinical population at age 12 years. Arch Gen Psychiatry. 2009;66(5):527-36.

9. Mackie CJ, Castellanos-Ryan N, Conrod PJ. Developmental trajectories of psychotic-like experiences across adolescence: impact of victimization and substance use. Psychol Med. 2010:1-12.

10. Arseneault L, Cannon M, Fisher HL, Polanczyk G, Moffitt TE, Caspi A. Childhood trauma and children's emerging psychotic symptoms: a genetically sensitive longitudinal cohort study. Am J Psychiatry. 2011;168:65-72.

11. Spauwen J, Krabbendam L, Lieb R, Wittchen H-U, van Os J. Impact of psychological trauma on the development of psychotic symptoms: relationship with psychosis proneness. Br J Psychiatry. 2006;188:527-33.

12. Elklit A, Shevlin M. Female sexual victimization predicts psychosis: a case-control study based on the Danish registry system. Schizophr Bull. 2011;37(6):1305-10.

13. Fisher H, Morgan C, Dazzan P, Craig TK, Morgan K, Hutchinson G, et al. Gender differences in the association between childhood abuse and psychosis. Br J Psychiatry. 2009;194(4):319-25.

14. Whitfield CL, Dube SR, Felitti VJ, Anda RF. Adverse childhood experiences and hallucinations. Child Abuse Negl. 2005;29(7):797-810.

15. Lataster T, van Os J, Drukker M, Henquet C, Feron F, Gunther N, et al. Childhood victimisation and developmental expression of non-clinical delusional ideation and hallucinatory experiences: victimisation and non-clinical psychotic experiences. Soc Psychiatry Psychiatr Epidemiol. 2006;41(6):423-8.

16. Scott J, Chant D, Andrews G, Martin G, McGrath J. Association between trauma exposure and delusional experiences in a large community-based sample. Br J Psychiatry. 2007;190:339-43.

17. Shevlin M, Dorahy MJ, Adamson G. Trauma and psychosis: an analysis of the National Comorbidity Survey. Am J Psychiatry. 2007;164(1):166-9.

18. Kelleher I, Harley M, Lynch F, Arseneault L, Fitzpatrick C, Cannon M. Associations between childhood trauma, bullying and psychotic symptoms among a school-based adolescent sample. Br J Psychiatry. 2008;193(5):378-82.

19. Shevlin M, Houston JE, Dorahy MJ, Adamson G. Cumulative traumas and psychosis: an analysis of the national comorbidity survey and the British Psychiatric Morbidity Survey. Schizophr Bull. 2008;34(1):193-9.

20. Freeman D, Fowler D. Routes to psychotic symptoms: trauma, anxiety and psychosis-like experiences. Psychiatry Res. 2009;169(2):107-12.

21. Shergill SS, Van Os J, Murray RM. Schizophrenia and depression: what is their relationship? In: Keck PE, 
editor. Managing the depressive symptoms of schizophrenia. London: Science Press; 1999. p. 12-29.

22. Hanssen M, Peeters F, Krabbendam L, Radstake S, Verdoux H, van Os J. How psychotic are individuals with non-psychotic disorders? Soc Psychiatry Psychiatr Epidemiol. 2003;38(3):149-54.

23. Varghese D, Scott J, Welham J, Bor W, Najman J, O'Callaghan M, et al. Psychotic-like experiences in major depression and anxiety disorders: a population-based survey in young adults. Schizophr Bull. 2011;37(2): 389-93.

24. van Rossum I, Lieb R, Wittchen H-U, van Os J. Affective dysregulation and reality distortion: a 10-year prospective study of their association and clinical relevance. Schizophr Bull. 2011;37(3):561-71.

25. Argyropoulos SV, Landau S, Kalidindi S, Toulopoulou T, Castle DJ, Murray RM, et al. Twins discordant for schizophrenia: psychopathology of the non-schizophrenic co-twins. Acta Psychiatr Scand. 2008;118(3):214-9.

26. Mortensen PB, Pedersen MG, Pedersen CB. Psychiatric family history and schizophrenia risk in Denmark: which mental disorders are relevant? Psychol Med. 2010;40(2):201-10.

27. Lardinois M, Lataster T, Mengelers R, Van Os J, Myin-Germeys I. Childhood trauma and increased stress sensitivity in psychosis. Acta Psychiatr Scand. 2011;123(1):28-35.

28. Kendler KS, Prescott CA. Genes, environment, and psychopathology. Understanding the causes of psychiatric and substance use disorders. New York: Guilford Press; 2006.

29. Wichers M, Schrijvers D, Geschwind N, Jacobs N, Myin-Germeys I, Thiery E, et al. Mechanisms of geneenvironment interactions in depression: evidence that genes potentiate multiple sources of adversity. Psychol Med. 2009;39(7):1077-86.

30. Wichers M, Myin Germeys I, Jacobs N, Peeters F, Kenis G, Derom C, et al. Genetic risk of depression and stress-induced negative affect in daily life. Br J Psychiatry. 2007;191:218-23.

31. Gottesman II, Gould TD. The endophenotype concept in psychiatry: etymology and strategic intentions. Am J Psychiatry. 2003;160(4):636-45.

32. Lataster T, Wichers M, Jacobs N, Mengelers R, Derom C, Thiery E, et al. Does reactivity to stress cosegregate with subclinical psychosis? A general population twin study. Acta Psychiatr Scand. 2009;119(1):45-53.

33. Myin-Germeys I, van Os J. Stress-reactivity in psychosis: evidence for an affective pathway to psychosis. Clin Psychol Rev. 2007;27(4):409-24.

34. Glaser JP, van Os J, Portegijs PJ, Myin Germeys I. Childhood trauma and emotional reactivity to daily life stress in adult frequent attenders of general practitioners. J Psychosom Res. 2006;61(2):229-36.

35. Garety PA, Kuipers E, Fowler D, Freeman D, Bebbington PE. A cognitive model of the positive symptoms of psychosis. Psychol Med. 2001;31(2):189-95.

36. Freeman D. Suspicious minds: the psychology of persecutory delusions. Clin Psychol Rev. 2007;27(4):42557.

37. van Os J, Verdoux H, Maurice-Tison S, Gay B, Liraud F, Salamon R, et al. Self-reported psychosis-like symptoms and the continuum of psychosis. Soc Psychiatry Psychiatr Epidemiol. 1999;34(9):459-63.

38. van Os J, Linscott RJ, Myin-Germeys I, Delespaul P, Krabbendam L. A systematic review and meta-analysis of the psychosis continuum: evidence for a psychosis proneness-persistence-impairment model of psychotic disorder. Psychol Med. 2009;39(2):179-95.

39. Myin-Germeys I, Krabbendam L, Delespaul PAEG, van Os J. Sex differences in emotional reactivity to daily life stress in psychosis. J Clin Psychiatry. 2004;65(6):805-9.

40. Kendler KS, Thornton LM, Prescott CA. Gender differences in the rates of exposure to stressful life events and sensitivity to their depressogenic effects. Am J Psychiatry. 2001;158(4):587-93.

41. Loos R, Derom C, Vlietinck R, Derom R. The East Flanders Prospective Twin Survey (Belgium): a population-based register. Twin Res. 1998;1(4):167-75.

42. Derom CA, Vlietinck RF, Thiery EW, Leroy FO, Fryns JP, Derom RM. The East Flanders Prospective Twin Survey (EFPTS). Twin Res Hum Genet. 2006;9(6):733-8.

43. Bernstein DP, Fink L, Handelsman L, Foote J, Lovejoy M, Wenzel K, et al. Initial reliability and validity of a new retrospective measure of child abuse and neglect. Am J Psychiatry. 1994;151(8):1132-6.

44. Bernstein DP, Ahluvalia T, Pogge D, Handelsman L. Validity of the Childhood Trauma Questionnaire in an 
adolescent psychiatric population. J Am Acad Child Adolesc Psychiatry. 1997;36(3):340-8.

45. Arntz A, Wessel I. Jeugd Trauma Vragenlijst [Dutch version of the Childhood Trauma Questionnaire]. Maastricht, the Netherlands: Author; 1996.

46. Bernstein DP, Stein JA, Newcomb MD, Walker E, Pogge D, Ahluvalia T, et al. Development and validation of a brief screening version of the Childhood Trauma Questionnaire. Child Abuse Negl. 2003;27(2):169-90.

47. First M, Spitzer R, Gibbon M, Williams J. SCID-I. Structured clinical interview for DSM-IV Axis I disorders [SCID I. Gestructureerd klinisch interview voor DSM-IV As-I stoornissen]. Lisse, the Netherlands: Swets \& Zeitlinger; 1998.

48. Arrindel W, Ettema J. SCL-90. Een multidimensionele psychopathologie indicator [The SCL90. A multidimensional instrument for the assessment of psychopathology]. Lisse: the Netherlands: Swets \& Zeitlinger; 1986.

49. Derogatis LR. SCL90: administration, scoring and procedures manual-1 for the revised edition. Baltimore, MD: John Hopkins School of Medicine; 1977.

50. Stefanis NC, Hanssen M, Smirnis NK, Avramopoulos DA, Evdokimidis IK, Stefanis CN, et al. Evidence that three dimensions of psychosis have a distribution in the general population. Psychol Med. 2002;32(2):347-58.

51. Konings M, Bak M, Hanssen M, van Os J, Krabbendam L. Validity and reliability of the CAPE: A self-report instrument for the measurement of psychotic experiences in the general population. Acta Psychiatr Scand. 2006;114(1):55-61.

52. Peters ER, Joseph SA, Garety PA. Measurement of delusional ideation in the normal population: introducing the PDI (Peters et al. Delusions Inventory). Schizophr Bull. 1999;25(3):553-76.

53. Csikszentmihalyi M, Larson R. Validity and reliability of the Experience-Sampling Method. J Nerv Ment Dis. 1987;175(9):526-36.

54. de Vries MW. The experience of psychopathology: investigating mental disorders in their natural settings. Cambridge: Cambridge University Press; 1992.

55. Delespaul P. Assessing schizophrenia in daily life: the experience sampling method. Maastricht, the Netherlands: Maastricht University Press; 1995.

56. Myin-Germeys I, Oorschot M, Collip D, Lataster J, Delespaul P, van Os J. Experience sampling research in psychopathology: opening the black box of daily life. Psychol Med. 2009;39:1533-47.

57. Simons CJP, Wichers M, Derom C, Thiery E, Myin-Germeys I, Krabbendam L, et al. Subtle gene-environment interactions driving paranoia in daily life. Genes Brain Behav. 2009;8(1):5-12.

58. Palmier-Claus JE, Myin-Germeys I, Barkus E, Bentley L, Udachina A, Delespaul PAEG, et al. Experience sampling research in individuals with mental illness: reflections and guidance. Acta Psychiatr Scand. 2011;123(1):12-20.

59. Jacobs N, Nicolson NA, Derom C, Delespaul P, van Os J, Myin-Germeys I. Electronic monitoring of salivary cortisol sampling compliance in daily life. Life Sci. 2005;76(21):2431-43.

60. Kendler KS, Kessler RC, Walters EE, MacLean C, Neale MC, Heath AC, et al. Stressful life events, genetic liability, and onset of an episode of major depression in women. Am J Psychiatry. 1995;152(6):833-42.

61. StataCorp. Stata Statistical Software: Release 11. College Station, TX, USA: Statacorp LP; 2009.

62. Clayton D, Hill M. Wald tests. In: Clayton D, Hill M, editors. Statistical Models in Epidemiology. Oxford: Oxford Science Publications; 1993. p. 101-2.

63. Bijl RV, Ravelli A, van Zessen G. Prevalence of psychiatric disorder in the general population: results of the Netherlands Mental Health Survey and Incidence Study (NEMESIS). Soc Psychiatry Psychiatr Epidemiol. 1998;33(12):587-95.

64. Maier W, Lichtermann D, Minges J, Hallmayer J, Heun R, Benkert O, et al. Continuity and discontinuity of affective disorders and schizophrenia. Results of a controlled family study. Arch Gen Psychiatry. 1993;50(11):871-83.

65. Häfner H, an der Heiden W, Maurer K. Evidence for separate diseases?: Stages of one disease or different combinations of symptom dimensions? Eur Arch Psychiatry Clin Neurosci. 2008;258 Suppl 2:85-96.

66. Häfner H, Maurer K, Trendler G, an der Heiden W, Schmidt M, Könnecke R. Schizophrenia and depression: challenging the paradigm of two separate diseases. A controlled study of schizophrenia, depression and 
healthy controls. Schizophr Res. 2005;77(1):11-24.

67. Owens DGC, Johnstone EC. Precursors and prodromata of schizophrenia: findings from the Edinburgh High Risk Study and their literature context. Psychol Med. 2006;36(11):1501-14.

68. Wichers M, Geschwind N, Jacobs N, Kenis G, Peeters F, Derom C, et al. Transition from stress sensitivity to a depressive state: longitudinal twin study. Br J Psychiatry. 2009;195(6):498-503.

69. Krabbendam L, Myin-Germeys I, Bak M, van Os J. Explaining transitions over the hypothesized psychosis continuum. Aust N Z J Psychiatry. 2005;39(3):180-6.

70. Krabbendam L, van Os J. Affective processes in the onset and persistence of psychosis. Eur Arch Psychiatry Clin Neurosci. 2005;255(3):185-9.

71. Verdoux H, van Os J, Maurice-Tison S, Gay B, Salamon R, Bourgeois ML. Increased occurrence of depression in psychosis-prone subjects: a follow-up study in primary care settings. Compr Psychiatry. 1999;40(6):462-8.

72. van Os J. A salience dysregulation syndrome. Br J Psychiatry. 2009;194(2):101-3.

73. van Os J. 'Salience syndrome' replaces 'schizophrenia' in DSM-V and ICD-11: psychiatry's evidence-based entry into the 21st century? Acta Psychiatr Scand. 2009;120(5):363-72.

74. Birchwood M. Pathways to emotional dysfunction in first-episode psychosis. Br J Psychiatry. 2003;182:373-5.

75. Allen P, Freeman D, McGuire P, Garety P, Kuipers E, Fowler D, et al. The prediction of hallucinatory predisposition in non-clinical individuals: examining the contribution of emotion and reasoning. $\mathrm{Br} \mathrm{J}$ Clin Psychol. 2005;44(1):127-32.

76. Garety PA, Bebbington P, Fowler D, Freeman D, Kuipers E. Implications for neurobiological research of cognitive models of psychosis: a theoretical paper. Psychol Med. 2007;37(10):1377-91.

77. Garety PA, Kuipers E, Fowler D, Freeman D, Bebbington PE. A cognitive model of the positive symptoms of psychosis. Psychol Med. 2001;31(2):189-95.

78. Baker CA, Morrison AP. Cognitive processes in auditory hallucinations: attributional biases and metacognition. Psychol Med. 1998;28(5):1199-208.

79. Freeman D, Garety PA. Worry, worry processes and dimensions of delusions: an exploratory investigation of a role for anxiety processes in the maintenance of delusional distress. Behav Cogn Psychother. 1999;27(1):47-62.

80. Morrison AP, Baker CA. Intrusive thoughts and auditory hallucinations: a comparative study of intrusions in psychosis. Behav Res Ther. 2000;38(11):1097-106.

81. Birchwood M, Chadwick P. The omnipotence of voices: testing the validity of a cognitive model. Psychol Med. 1997;27(6):1345-53.

82. Green C, Garety PA, Freeman D, Fowler D, Bebbington P, Dunn G, et al. Content and affect in persecutory delusions. Br J Clin Psychol. 2006;45(4):561-77.

83. Corcoran C, Walker E, Huot R, Mittal V, Tessner K, Kestler L, et al. The stress cascade and schizophrenia: etiology and onset. Schizophr Bull. 2003;29:671-692.

84. Heim C, Newport DJ, Mletzko T, Miller AH, Nemeroff CB. The link between childhood trauma and depression: insights from HPA axis studies in humans. Psychoneuroendocrinology. 2008;33(6):693-710.

85. Phillips LJ, McGorry PD, Garner B, Thompson KN, Pantelis C, Wood SJ, et al. Stress, the hippocampus and the hypothalamic-pituitary-adrenal axis: implications for the development of psychotic disorders. Aust N Z J Psychiatry. 2006;40(9):725-41.

86. Collip D, Myin Germeys I, Van Os J. Does the concept of "sensitization" provide a plausible mechanism for the putative link between the environment and schizophrenia? Schizophr Bull. 2008;34(2):220-5.

87. Boks MPM, Leask S, Vermunt JK, Kahn RS. The structure of psychosis revisited: the role of mood symptoms. Schizophr Res. 2007;93(1-3):178-85.

88. Verdoux H, van Os J. Psychotic symptoms in non-clinical populations and the continuum of psychosis. Schizophr Res. 2002;54(1-2):59-65.

89. van Os J, Hanssen M, Bijl RV, Ravelli A. Strauss (1969) revisited: a psychosis continuum in the general population? Schizophr Res. 2000;45(1-2):11-20.

90. Van Os J, Gilvarry C, Bale R, Van Horn E, Tattan T, White I, et al. A comparison of the utility of dimensional 
and categorical representations of psychosis. UK700 Group. Psychol Med. 1999;29(3):595-606.

91. Krabbendam L, Myin-Germeys I, De Graaf R, Vollebergh W, Nolen WA, Iedema J, et al. Dimensions of depression, mania and psychosis in the general population. Psychol Med. 2004;34(7):1177-86.

92. Cougnard A, Marcelis M, Myin-Germeys I, De Graaf R, Vollebergh W, Krabbendam L, et al. Does normal developmental expression of psychosis combine with environmental risk to cause persistence of psychosis? A psychosis proneness-persistence model. Psychol Med. 2007;37(4):513-27.

93. Allardyce J, Suppes T, Van Os J. Dimensions and the psychosis phenotype. Int J Methods Psychiatr Res. 2007;16 (Suppl.):S34-S40.

94. Olfson M, Lewis-Fernandez R, Weissman MM, Feder A, Gameroff MJ, Pilowsky D, et al. Psychotic symptoms in an urban general medicine practice. Am J Psychiatry. 2002;159(8):1412-9. 


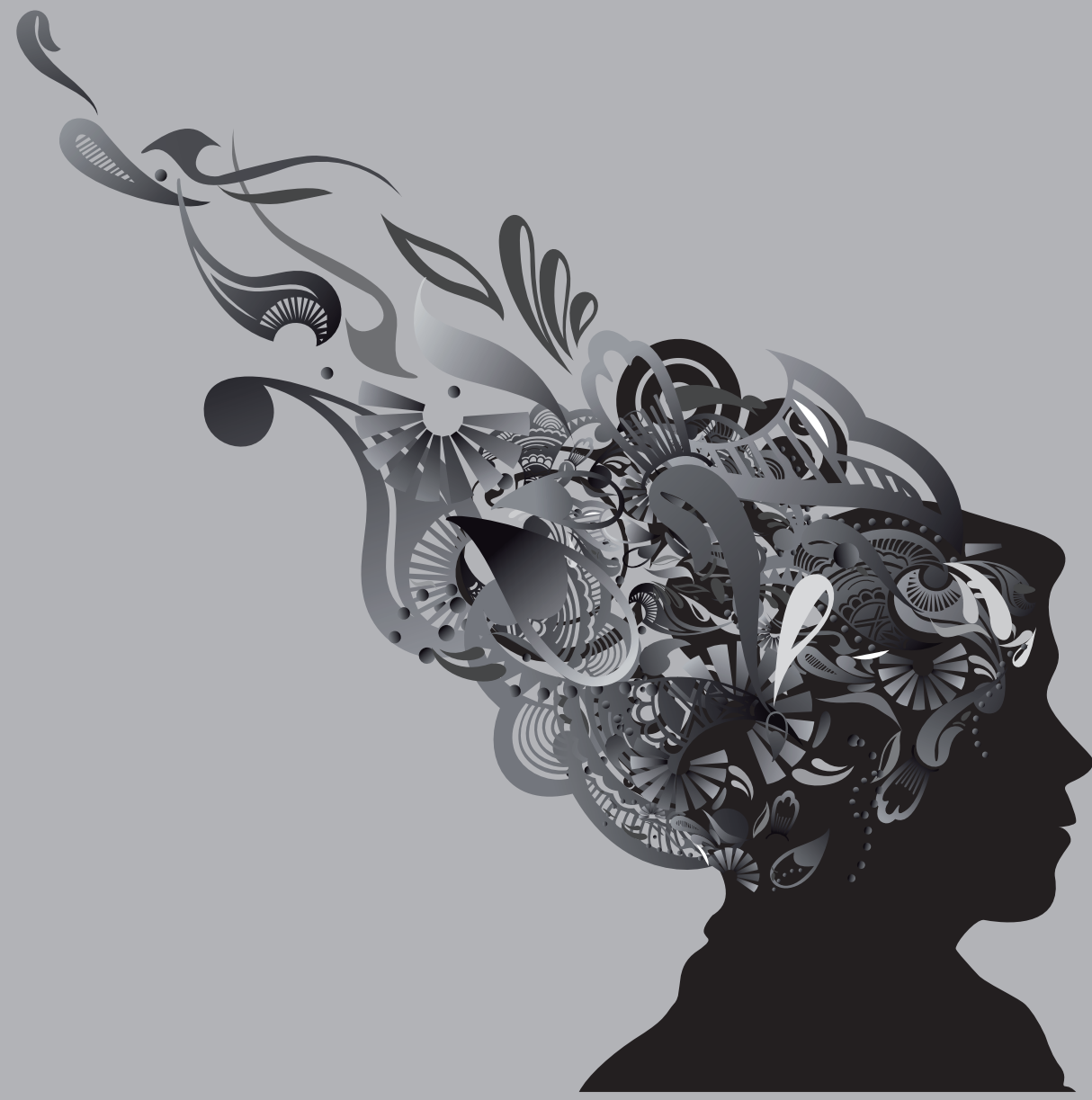




\section{CHAPTER 3}

\section{Time-lagged moment-to-moment interplay between negative affect and paranoia: new}

\section{insights in the affective pathway to psychosis}

I. Kramer ${ }^{1,2}$, CJP. Simons ${ }^{1,2}$, JTW. Wigman ${ }^{2}$, D. Collip ${ }^{2}$, N. Jacobs ${ }^{2,3}$, C. Derom ${ }^{4}$, E .Thiery ${ }^{5}$, J. van Os ${ }^{2,6}$, I. Myin-Germeys ${ }^{2}$, M. Wichers ${ }^{2}$

${ }^{1}$ GGzE, Institute of Mental Health Care Eindhoven and the Kempen, Eindhoven, the Netherlands

${ }^{2}$ Department of Psychiatry and Psychology, South Limburg Mental Health Research and Teaching Network, EURON, Maastricht University, the Netherlands ${ }^{3}$ Faculty of Psychology, Open University of the Netherlands, Heerlen, the Netherlands

${ }^{4}$ Department of Human Genetics, University Hospital Gasthuisberg, Katholieke Universiteit Leuven, Leuven, Belgium

${ }^{5}$ Dept of Neurology, Ghent University Hospital, Ghent, Belgium ${ }^{6}$ King's College London, Department of Psychosis Studies, Institute of Psychiatry, London, UK 


\section{Abstract}

Background: Evidence suggests that affect plays a role in the development of psychosis but the underlying mechanism requires further investigation. This study examines the moment-to-moment dynamics between negative affect (NA) and paranoia prospectively in daily life.

Methods: A female general population sample $(\mathrm{n}=515)$ participated in an experience sampling study. Time-lagged analyses between increases in momentary NA and subsequent momentary paranoia were examined. The impact of childhood adversity, stress sensitivity (impact of momentary stress on momentary NA), and depressive symptoms on these time-lagged associations, as well as associations with follow-up self-reported psychotic symptoms (Community Assessment of Psychic Experiences and the Symptom Checklist -90-Revised) were investigated.

Results: Moments of NA increase resulted in a significant increase in paranoia over 180 subsequent minutes. Both stress sensitivity and depressive symptoms impacted on the transfer of NA to paranoia. Stress sensitivity moderated the level of increase in paranoia during the initial NA increase, while depressive symptoms increased persistence of paranoid feelings from moment to moment. Momentary paranoia responses to NA increases were associated with follow-up psychotic symptoms.

Conclusions: Examination of micro-level momentary experience may thus yield new insights into the mechanism underlying co-occurrence of altered mood states and psychosis. Knowledge of the underlying mechanism is required in order to determine source and place where remediation should occur. 


\section{Introduction}

Recent evidence indicates that the phenotype of psychosis is dimensional in nature and that the more frequent subclinical psychotic experiences share demographic, etiological and familial factors with the more severe clinical psychotic symptoms (1). Furthermore, persistence of subclinical experiences of psychosis has been shown to predict transition to clinical symptoms and help-seeking behavior (2).

Psychotic symptoms tend to occur more often in a context of negative mood states, at both the subclinical and the clinical level of expression $(3,4)$. In a large general population study, psychotic experiences were reported by $27 \%$ of the individuals with an anxiety disorder or depression (5). Furthermore, the presence of psychotic experiences and clinically relevant psychotic symptoms in these individuals was progressively more likely with greater levels of manic or depressive symptoms (4). Also, depression and psychosis share a number of important risk factors such as childhood adversity (6-8), social dysfunction $(9,10)$, social defeat experiences $(11)$ and daily life stress sensitivity (12-15). Together, this suggests that affective alterations may be relevant to the development of both disorders (15). Support for this notion comes from a recent twin study (16) which showed a role for childhood adversity, daily life stress sensitivity and current depressive symptoms in the shared pathway towards depression and psychotic symptoms. Also, previous studies demonstrated that the above risk factors enhance the experience of negative affect $(6,12-15)$, suggesting that negative affect (NA) could be the key element that increases risk for both depression and psychosis. Cohort studies can show associations between symptoms domains; however, they are less suitable to find out why significant factors on the causal pathway contribute to shared risk for both depression and psychosis. To further disentangle the underlying mechanisms, we may have to zoom in to the level of moment-to-moment affective dynamics connecting NA and subclinical feelings of paranoia. This shift from the epidemiological (macro-) level to the micro-level of hour-by-hour tracking of emotional dynamics may help explain whether and how exactly affective experiences impact on risk for psychosis (17), and how shared risk factors such as childhood adversity, stress sensitivity and depressive symptoms may impact on dynamics between NA and subclinical paranoid ideation.

The Experience Sampling Method (ESM) is a technology that allows for prospective tracking of affective and psychotic experiences from moment to moment $(18,19)$, and thus suitable to examine how within-person change in one variable impacts on change in the other variable later on the same day (20).

Therefore, the aim of the current study was to use ESM to examine prospectively (1) how moment-to-moment increase in NA impacts on subsequent feelings of paranoia, (2) whether this effect is moderated by childhood adversity, daily life stress sensitivity and 
depressive symptoms, and finally (3) whether the dynamics between NA and paranoia are moderated by follow-up course of psychotic symptoms.

\section{Methods}

\section{Sample}

Data were derived from 621 female individuals (576 twins and 45 non-twin sisters), who were part of a longitudinal, general population twin study on genes, stress sensitivity and depression. Subjects were aged 18-61 years. They were recruited by mail (for details see Jacobs and colleagues (21)) from the East Flanders Prospective Twin Survey (EFPTS) and from birth registers of Flemish municipalities in Belgium. The EFPTS population-based survey has prospectively recorded all multiple births in the province of East Flanders since $1964(22,23)$. The project was approved by the local ethics committee. All participants gave written informed consent. Given evidence for qualitative differences in the type of environmental stressors that are associated with depression in men and women (24), the sample was female only.

\section{Procedure}

In this study, the Experience Sampling Method (ESM) was completed at baseline as well as additional measurements of psychopathology. Furthermore, participants completed four follow-up assessments (T1-T4) with measurements of psychopathology. The average number of days between baseline and T1 was 132, 91 between T1 and T2, 116 between T2 and T3 and 91 between T3 and T4. All interviews were administered by trained research psychologists or graduate psychological assistants.

\section{ESM assessment}

ESM is a momentary assessment technique to prospectively assess momentary experiences of subjects in their daily living environment $(13,18,19)$. Subjects received a digital wristwatch and a set of ESM self-assessment forms collected in a booklet for each day. The wristwatch was programmed to emit a signal (beep) at an unpredictable moment in each of ten 90-minute time blocks between 7:30 and 22:30, on five consecutive days, resulting in a maximum of 50 beeps per person. The study used a semi-random beep design to prevent anticipatory behavior of participants (for more methodological information, see previous ESM literature $(13,14,18))$.

Appraisals of minor daily stressful events and momentary feelings were measured. To measure ESM event-related stress, subjects were asked to report the most important event that happened between the current and the previous beep. This event was subsequently rated on a 7 -point bipolar scale (from $-3=$ very unpleasant, $0=$ neutral, 
to 3 = very pleasant). Event appraisals were thus available at each measurement. The scale was reversed so that higher scores represented a greater degree of dislike for the event (event stress). Stress sensitivity was defined as the effect of the (un)pleasantness appraisal of the most important event that had happened since the last beep on negative affect (NA) $6,12-14)$. Appraised events are thus compared for their level of NA. To create the variable stress sensitivity, the weighted mean NA was regressed on ESM event-related stress, resulting in a separate beta coefficient for each individual (see also Wichers and colleagues (13)).

Current affective states and feelings of paranoia were assessed as follows: 'at this moment I feel ..... The items assessing paranoia and other negative affective states were embedded in a list of adjectives comprising both negative and positive affective states. To prevent participants from reporting an overall negative affective experience, positive and negative items were alternated.

Factor analysis identified a single factor representing NA. The items 'insecure', 'lonely', 'anxious', 'low' and 'guilty' -weighted for their factor loadings- were averaged to form the measurement of NA (respective loadings were: $0.71,0.60,0.66,0.68,0.61$ ).

For the current purpose, paranoia was assessed separately with the adjective 'suspicious' conforming to previous studies $(20,25)$.

\section{Potential moderators}

Childhood adversity. Childhood adversity was measured at baseline (T0) using the shortened version Childhood Trauma Questionnaire (CTQ)(26, 27) comprising 25 items that assess five dimensions of childhood maltreatment: (1) physical abuse, (2) emotional abuse, (3) sexual abuse, (4) physical neglect, and (5) emotional neglect. At the request of Twin Registry, the four most explicit items concerning sexual and physical abuse were omitted, resulting in a 21-item self-report questionnaire. Items were scored on a scale of 1 (never true) to 5 (very often true). The sum score of all items was used as a continuous measure of childhood adversity.

Depressive symptoms. A continuous measure of depressive symptoms was obtained using the validated depression subscale (13 items) of the Symptom Checklist-90Revised (SCL-90-R)(28, 29). The SCL-90-R depression scores collected at baseline (T0) were used as a measure of current depressive symptoms.

Psychotic symptoms. Psychotic symptoms were measured in two ways. First, psychotic symptoms were measured with the SCL-90-R with the two validated subscales paranoid ideation (6 items) and psychoticism (10 items) $(28,29)$. The SCL-90-R was administered 
at all T0-T4 measurement occasions. Baseline psychosis score was assessed at T0. Follow-up SCL-90-R psychosis score was calculated as the mean over the measurements at T1, T2, T3, and T4. The mean of the four follow-up measurements was used to obtain reliable assessments of follow-up psychotic symptoms.

Second, psychotic experiences were assessed with the Community Assessment of Psychic Experiences (CAPE)(30). The CAPE is a 42-item validated self-report questionnaire to assess psychopathological experiences in the general population (30). Items resemble positive symptoms of schizophrenia (20 items), negative symptoms (14 items), and depressive symptoms ( 8 items). The CAPE was administered 3 times, at baseline T0, and at T2 and T4. A baseline psychosis score was constructed using the positive symptom dimension at T0; a follow-up psychosis score was constructed using the mean of this dimension over the follow-ups (T2 and T4).

\section{Statistical analysis}

Analyses were carried out in STATA 11.0 (31). As ESM measurements of NA and paranoia were available for 5 days with a maximum of 10 measurements per day per person with an average $90 \mathrm{~min}$ in between, the data were ideally suited to explore the dynamic within-subject temporal associations between NA and paranoia. Within-subject fluctuations of paranoia following a within-subject increase in NA were examined. Within-subject increase in NA was defined as reporting higher levels of negative affect compared with the beep before. An advantage of measurements of within-subject change is that all potential between-person differences in scoring tendencies on the Likert scale are eliminated. To examine paranoia fluctuations following NA increase, all the beep moments with a within-subject increase in NA, compared with the beep before, were selected. Variables were created representing paranoia at beep moments before, during, and following the increase in NA (i.e. paranoia at $t-1, t, t+1, t+2, t+3$, $t+4, t+5$ ). Thus, multiple time-lags were examined to create a fine-grained 'film' of changes in paranoia relating to an increase in NA in daily life. The data were reshaped in long format with a new 'time' variable representing the distance in beeps from the moment of 'increase in NA'. In this way, a multilevel regression analysis with time as the independent and paranoia as the dependent variable could be carried out. The time value $\mathrm{t}-1$ (one beep before the increase in NA) was taken as reference value.

The following models were used: The main effect of NA increase on paranoia over $\mathrm{t}-1$ to $t+5$ was examined by regressing paranoia following the NA increase on time. To test the hypothesis that putative risk factors moderate the effect of time on paranoia following NA increase, paranoia was regressed on the interaction between the risk factor and time. This was done separately for each of the three risk factors, by regressing paranoia on the relevant 2-way interaction (respectively: 'childhood adversity x time', 'stress sensitivity 
$\mathrm{x}$ time', and 'current depressive symptoms $\mathrm{x}$ time'). To facilitate interpretation of the 2-way interaction analyses, dose-response relationships were graphically displayed. These were assessed by dividing the distributions of the continuous risk factor variables (childhood adversity, stress sensitivity, and current depressive symptoms, respectively) by their tertiles. Stratified effect sizes were calculated by linear combination of the relevant variables in the model containing the interaction, using the STATA LINCOM routine. Main effects and interactions were evaluated statistically with Wald tests. Independence of the different interactions was examined by simultaneously entering all three interactions into the regression model.

Finally, analyses were conducted to examine whether moment-to-moment NAparanoia dynamics were associated with follow-up course of psychotic symptoms. In this analysis, it was examined whether the effect of time (in beeps) on paranoia was moderated by follow-up SCL-90-R and CAPE psychosis score. These analyses were corrected for baseline psychosis score.

ESM data have a hierarchical structure in which ESM observations (level 1) are clustered within participants (level 2). In addition, in the current data set participants (level 2) were clustered within twin pairs (level 3). Data were, therefore, analyzed using multilevel random regression analysis with the XTMIXED module. Analyses were adjusted for the absolute amount of NA increase, because the amount of emotional change may confound the subsequent response. Continuous variables were standardized to obtain comparable standardized effect sizes.

\section{Results}

\section{Subjects characteristics}

Of the total subject sample of 621 white females, 610 participated in the ESM procedure. Thirty-one subjects were excluded because they missed more than $30 \%$ of valid ESM self-reports (18). This resulted in a subject sample of 579 female subjects. Another 64 individuals were excluded because they did not demonstrate an increase in NA over the total ESM period, resulting in a sample of 515 individuals. An additional 8, 24 and 7 individuals had missing values for childhood adversity, stress sensitivity and current depressive symptoms, respectively. Furthermore, 2 and 53 individuals had missing values at all follow-up measurements of SCL-90-R and CAPE psychosis scores, respectively.

Mean age of the 515 subjects was 27.7 years (SD = 8.3, range 18-61). Sixty-four percent had a college or university degree, $34 \%$ completed secondary education, and $1 \%$ had primary education only. The majority were currently employed ( $60 \%$ employed, $37 \%$ students, $3 \%$ unemployed). At baseline, 66 subjects $(12.8 \%)$ had one or more 
Structured Clinical Interview for DSM-IV Axis I disorders (SCID-I) psychotic symptoms. Twenty-five subjects (4.9\%) had a current major depression and 86 (16.7\%) had a history of major depression. Baseline mean item scores for SCL-90-R depression, SCL90-R psychosis, CAPE psychosis, and childhood adversity were $1.6(\mathrm{SD}=.62), 1.4(\mathrm{SD}=$ $.41), 1.3(\mathrm{SD}=.21)$, and $1.7(\mathrm{SD}=.63)$, respectively. Mean stress sensitivity score was .0 .2 $(\mathrm{SD}=.29)$. Correlations between childhood adversity, stress sensitivity and current SCL90-R depression were low to moderate, ranging from .14 to .24 and .39.

A total of 37 valid beep reports were completed per individual. At the selected beep moments with a NA increase $\left(\mathrm{N}_{\text {obs }}=2906, \mathrm{n}_{\text {subj }}=515\right)$, the average reported NA (on a scale from 1 to 7 ) was $2.0(\mathrm{SD}=.86)$, average NA increase was .66 ( $\mathrm{SD}=.60)$, and average paranoia was $1.4(\mathrm{SD}=1.0)$. Average reported paranoia before and following increase in NA varied from 1.2 to 1.3. Correlations between paranoia and NA items varied from .11 to .31. Mean follow-up SCL-90-R psychosis item scores ranged from 1 to 3.1 (median of 1.2). Mean follow-up CAPE psychosis item scores ranged from 1 to 1.9 (median of 1.2).

\section{NA and paranoia daily dynamics}

Paranoia was significantly increased at moments following the increase in NA. Up to four beep moments later (at $t, t+1( \pm 90 \mathrm{~min}), t+2( \pm 180 \mathrm{~min})$, and $t+4( \pm 360 \mathrm{~min})$ ), the increase in paranoia, compared with paranoia at $\mathrm{t}-1$, was statistically significant (see figure 1).

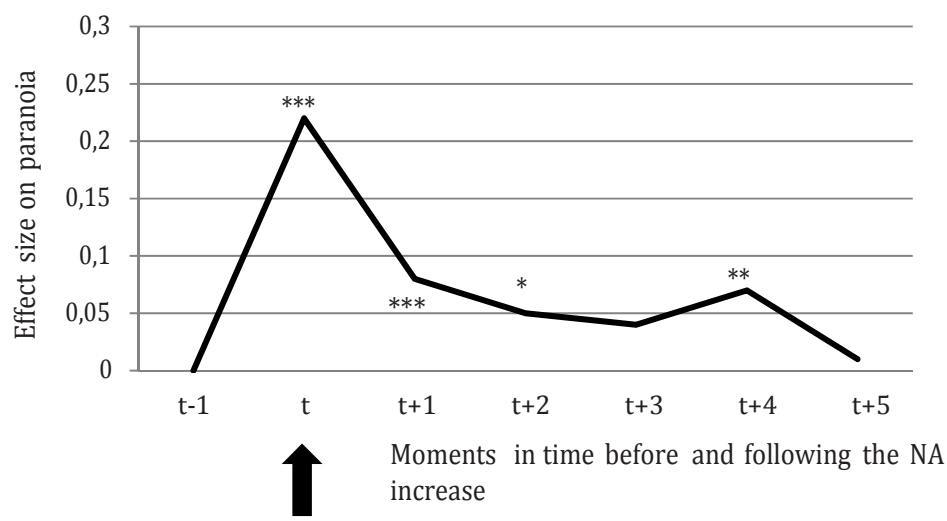

NA increase

*** $\mathrm{P} \leq .001, * * \mathrm{P} \leq .01,{ }^{*} \mathrm{P} \leq .05$

Figure 1 | Paranoia fluctuations over time following negative affect (NA) increase. Levels of paranoia at the different beep moments were tested for significance compared with their baseline level (at $t-1)$. 


\section{Moderation of NA-paranoia dynamics by risk factors}

\section{Childhood adversity}

Childhood adversity positively moderated the effect of increase in NA on subsequent paranoia levels. For two consecutive moments $(t$, and $t+1$; up to $\pm 90 \mathrm{~min}$ following the increase in NA), the interaction effect of 'time $x$ childhood adversity' on paranoia was significant (table 1), with greatest effect sizes for those in the highest tertile group of childhood adversity (figure $2 \mathrm{a}$ ).

Table 1 I Moderation by risk factors of the effect of time (before and following increase in NA) on paranoia.

\begin{tabular}{|c|c|c|c|c|c|c|c|c|c|c|c|c|c|c|c|c|c|c|}
\hline \multirow[t]{2}{*}{ Effect on paranoia } & \multicolumn{3}{|c|}{$\begin{array}{l}\text { Childhood } \\
\text { adversity } \\
\text { x time }^{\text {a }} \\
\end{array}$} & \multicolumn{3}{|c|}{$\begin{array}{l}\text { Childhood } \\
\text { adversity } \\
\text { x time } \\
\end{array}$} & \multicolumn{3}{|c|}{$\begin{array}{l}\text { Stress } \\
\text { sensitivity } \\
\text { x time }^{\mathrm{a}} \\
\end{array}$} & \multicolumn{3}{|c|}{$\begin{array}{l}\text { Stress } \\
\text { sensitivity } \\
\mathrm{x} \mathrm{time}^{\mathrm{a}, \mathrm{b}}\end{array}$} & \multicolumn{3}{|c|}{$\begin{array}{l}\text { Depression } \\
x_{\text {time }}{ }^{a}\end{array}$} & \multicolumn{3}{|c|}{$\begin{array}{l}\text { Depression } \\
\text { x time }^{\mathrm{a}, \mathrm{b}}\end{array}$} \\
\hline & $\beta$ & & n & $\beta$ & & $n$ & 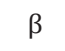 & & $\mathrm{p}$ & $\beta$ & & $n$ & $\beta$ & & $\mathrm{n}$ & $\Omega$ & & $p$ \\
\hline & .05 & & & & & & .07 & & & .06 & 7.5 & & 03 & 2.0 & 5 & .00 & 0.0 & 0 \\
\hline & 0 & & & & & & & & & & & & .08 & & & 2.0 & & .0 \\
\hline & .04 & & & & & & & & .79 & & & & & & .03 & .04 & & \\
\hline & 0 & & & & & & & & & 0 & & & .03 & & 1 & .02 & & \\
\hline & -.02 & & & & & & .03 & & .35 & .02 & & .45 & .02 & 0.4 & .54 & .02 & 0.4 & \\
\hline$+5: 5$ beeps later & .02 & 0.8 & .38 & .01 & & .88 & .02 & 0.4 & .54 & .01 & 0.1 & .81 & .05 & 2.4 & .12 & .04 & 1.2 & .2 \\
\hline
\end{tabular}

Note: All effects sizes of the interactions were standardized. Significant interaction effects are in bold. The reference category is the moment before the increase in negative affect (NA) $(t-1)$. Time difference between beeps is on average $90 \mathrm{~min}$.

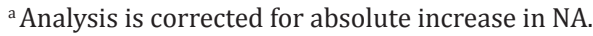

${ }^{\mathrm{b}}$ Full model includes all three 2-way interactions (childhood adversity $\mathrm{x}$ time, stress sensitivity $\mathrm{x}$ time, and depression $\mathrm{x}$ time).

\section{Stress sensitivity}

Stress sensitivity positively moderated the effect of an increase in NA on subsequent paranoia levels (see table 1 ). For two consecutive moments ( $t$, and $t+1$; up to $\pm 90 \mathrm{~min}$ following the increase in NA), the interaction effect of 'time $x$ stress sensitivity' on paranoia was significant, again with greatest effect sizes being apparent for those in the highest tertile group of stress sensitivity (figure $2 \mathrm{~b}$ ).

\section{Current depressive symptoms}

Current depressive symptoms positively moderated the effect of NA increase on subsequent paranoia levels (see table 1 ). For two consecutive moments $(t+1$, and $t+2$; from \pm 90 up to \pm 180 min following the increase in NA), the interaction effect between 
depressive symptoms and time on paranoia was significant, again with greatest effect sizes for those in the highest tertile group of current depressive symptoms (figure 2c).

Independence of effects of childhood adversity, stress sensitivity, and current depressive symptoms

When all three 2-way interaction variables were entered simultaneously in the model (table 1), the significance of 'childhood adversity (continuous) x time' on paranoia

A

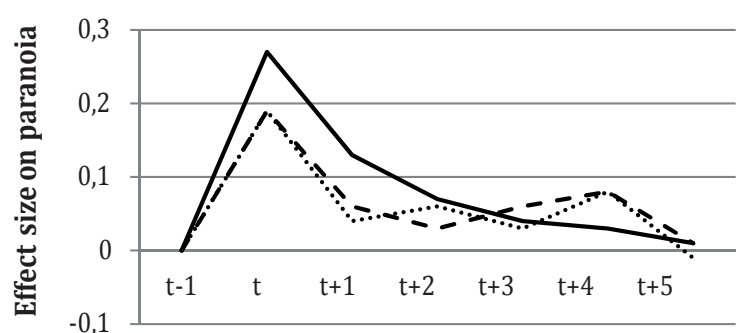

— Paranoia for high childhood adversity

- - - Paranoia for medium childhood adversity

........ Paranoia for low childhood adversity

Moments in time with respect to the NA increase

B

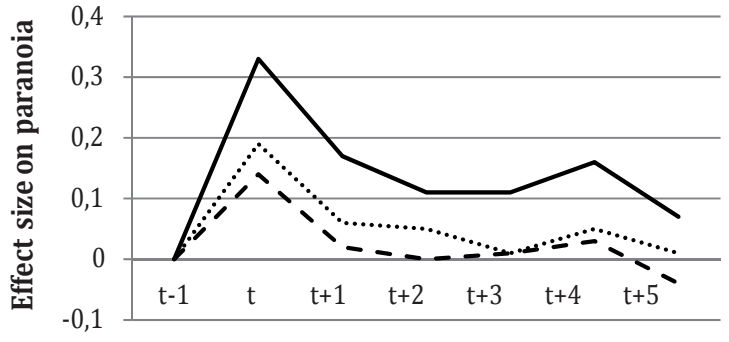

Paranoia for high stress
sensitivity

- - - Paranoia for medium stress sensitivity

Paranoia for low stress sensitivity

Moments in time with respect to the NA increase

C

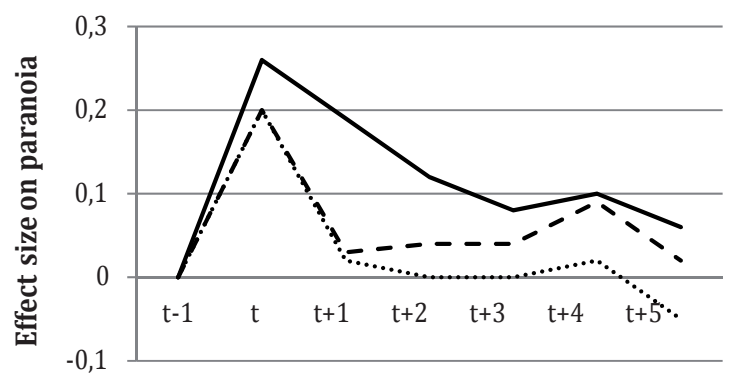

— Paranoia for high depressive symptoms

- - - Paranoia for medium depressive symptoms

Paranoia for low depressive symptoms

Moments in time with respect to the NA increase

Figure 2 | Effect of time on paranoia following negative affect (NA) increase stratified by level of childhood adversity (A), stress sensitivity (B), or current depressive symptoms (C), respectively. 
following the NA increase at both $t$ and $t+1$ was lost (8\% and 26\% drop in effect size, respectively). The moderation of the effect by stress sensitivity remained significant only at the moment of NA increase (effect sizes dropped $2 \%$ at $t$ and $16 \%$ at $t+1$, respectively). The moderation of the effect by 'current depressive symptoms' remained significant at $t+1$, but not at $t+2(9 \%$ and $11 \%$ drop in effect size, respectively).

The association between the paranoia response to NA increase in daily life and follow-up psychotic symptoms

Follow-up psychotic symptoms positively moderated the effect of NA increase on subsequent paranoia levels. For three consecutive moments $(t, t+1$, and $t+2$; up to \pm 180 min following the increase in NA), the interaction effect between mean follow-up SCL-90-R psychosis score and time following NA increase on paranoia was significant (table 2).

The interaction effect between mean follow-up CAPE psychosis and time following NA increase on paranoia was significant at four beep moments $(t, t+1, t+2$ and $t+5$; up to 450 min following the increase in NA) (see table 2).

Table 2 | Moderation of the effect of time (before and following increase in NA) on paranoia by follow-up psychotic symptoms

\begin{tabular}{lcccccc}
\hline Effect on paranoia & \multicolumn{2}{c}{ Follow-up SCL-90-R psychosis } & \multicolumn{3}{c}{ Follow-up CAPE psychosis } \\
& $\beta$ & $\chi^{2}$ & $\mathrm{P}$ & $\beta$ & $\chi^{2}$ & $\mathrm{P}$ \\
\hline $\mathrm{t}$ : Paranoia at NA increase & .08 & 14.1 & $\leq .001$ & .07 & 9.8 & .002 \\
$\mathrm{t}+$ 1: Paranoia 1 beep later & .10 & 15.5 & $\leq .001$ & .06 & 4.9 & .03 \\
$\mathrm{t}+2$ : Paranoia 2 beeps later & .05 & 4.0 & .05 & .06 & 4.2 & .04 \\
$\mathrm{t}+$ 3: Paranoia 3 beeps later & .04 & 2.5 & .11 & .03 & 1.1 & .30 \\
$\mathrm{t}+$ 4: Paranoia 4 beeps later & .04 & 1.7 & .19 & .06 & 3.5 & .06 \\
$\mathrm{t}+$ 5: Paranoia 5 beeps later & .06 & 3.4 & .07 & .08 & 5.4 & .02 \\
\hline
\end{tabular}

Note: CAPE, Community Assessment of Psychic Experiences; NA, negative affect; SCL-90-R, Symptom Checklist-90-Revised. All effects sizes of the interactions were standardized. Significant interaction effects are in bold. The reference category is the moment before the increase in NA ( $t-1)$. Time difference between beeps is on average $90 \mathrm{~min}$. All analyses were corrected for baseline levels of psychotic experiences.

\section{Discussion}

This study showed a subtle moment-to-moment dynamic interplay between NA and feelings of paranoia. An increase in NA resulted in a simultaneous increase in feelings of paranoia that remained significant up to $3 \mathrm{~h}$ afterward. Furthermore, childhood adversity, stress sensitivity and current depressive symptoms differentially affected the dynamic interplay between NA and paranoia, in terms of changes in increase and 
persistence of the paranoia response to the change in NA. Finally, individuals with higher SCL-90-R and CAPE follow-up psychotic symptoms showed increased transfer of NA to feelings of paranoia. Thus, paranoia following subtle everyday feelings of NA was associated with future course of symptoms, indicating that daily life micro dynamics between NA and paranoia are relevant for future symptomatology.

\section{Emotional dynamics: a differentiation between amplitude and persistence}

The current results illustrate how affective risk factors exert their effects at the microlevel of daily life experiences. Results demonstrated that childhood adversity, stress sensitivity, and current depressive symptoms all impacted on the dynamic interplay between NA and paranoia. However, the effect of childhood adversity was no longer significant when the interactions of all risk factors were entered into the model simultaneously. Because childhood adversity is known to increase stress sensitivity(6), variance previously explained by childhood adversity was likely explained by stress sensitivity in the latter model. Stress sensitivity and depressive symptoms, however, did show independent effects on the transfer of NA to paranoid feelings, but differed in the mechanism by which they impacted on this transfer. While stress sensitivity exerted an immediate effect, the effect of current depressive symptoms on paranoia had a delayed onset. Stress sensitivity increased the amplitude of the paranoia boost at the moment of NA increase, possibly reflecting altered attentional focus on negative valence $(32,33)$. Depressive symptoms, on the other hand, did not significantly influence the amplitude at the moment of NA increase, but affected the persistence of paranoia. Depressive symptoms thus increased the time that paranoid feelings remained present over the day. Alterations in rumination, a response-focused emotion regulation strategy, or alterations in capacity for cognitive reappraisal, an antecedent-focused emotion regulation strategy, may play a role in persistence of paranoid feelings in individuals with depressive symptoms (32-36). Epidemiological studies found that, at the macrolevel, persistence of psychotic symptoms is a strong risk factor for developing clinically relevant psychotic symptoms (2). It should be further investigated whether this is also true for psychotic experiences at the level of moment-to-moment persistence. This study showed that both stress sensitivity and depressive symptoms impacted on the dynamic interplay between NA and paranoia. This contrasts with a previous study (16), which showed that depressive symptoms, but not daily life stress sensitivity, was the final mediator on the pathway from childhood adversity to psychosis. However, it is possible that persistence of paranoia, associated here with depressive symptoms, has a stronger influence on future course than moment-specific higher levels of paranoia, associated here with stress sensitivity. 


\section{Insights into the affective pathway of psychosis: combining knowledge of the macro and the micro-levels of experience}

The current results provide further insights into the underlying mechanisms of the co-occurrence of affective symptomatology and psychosis (see figure 3). At the epidemiological (macro) level, it has been shown that 1) childhood adversity is a risk factor for both depressive and psychotic symptoms $(6,7), 2)$ childhood adversity predicts increased negative affective responses to stress in daily life $(6,15), 3)$ stress sensitivity in turn predicts depressive and psychotic symptoms $(13,14)$, and 4) depressive symptoms mediate the pathway from childhood adversity to psychotic symptoms (16). Furthermore, psychotic symptoms, especially when persistent, constitute a risk factor for later clinical symptoms, help-seeking behavior and, finally, the development of diagnosable psychotic disorder $(2,17)$. The shift to the micro-level in the current study adds insights into how and why risk factors for NA may impact on the later development of psychosis. It seems that at the momentary level NA may drive the experience of low-level paranoid feelings. Repetitively such increases in paranoia experiences following boosts of NA may sensitize individuals and build up to something more clinically relevant (37). This study showed indeed that the accumulation of everyday subtle paranoia following increase in NA predicted future course of psychotic symptoms. The findings of the current study thus support the idea that the smallest and most subtle emotional patterns and interaffective responses in daily life cumulatively may result in observable psychotic symptoms months later. Zooming into the microlevel may, therefore, constitute a way to uncover the smallest building blocks of the dimensional development of psychosis. Knowledge of these underlying mechanisms is highly relevant to get closer to the exact source and place where remediation should take place.

The findings should be interpreted in the light of current knowledge on emotion regulation, the processes by which humans modify negative and positive emotions with regard to their intensity, when they occur, how long they last, and how they are expressed (32). The "Process Model" by Gross $(32,33)$ is the leading model of emotion regulation. It proposes that emotions unfold as a multicomponential process, whereby a situation occurs (either external or internal) that is then attended to, giving rise to an appraisal of the situation's valence and motivational relevance, which results in a series of experiential, behavioral, and neurophysiological response changes. As discussed above, both antecedent-focused (situation selection, situation modification, attentional deployment, cognitive change/reappraisal) and response-focused (response modulation/affective suppression/rumination) emotion regulation strategies may be relevant in explaining the findings. Therefore, interventions teaching individuals to use emotion regulation strategies, as developed for use in anxiety disorders and depression 


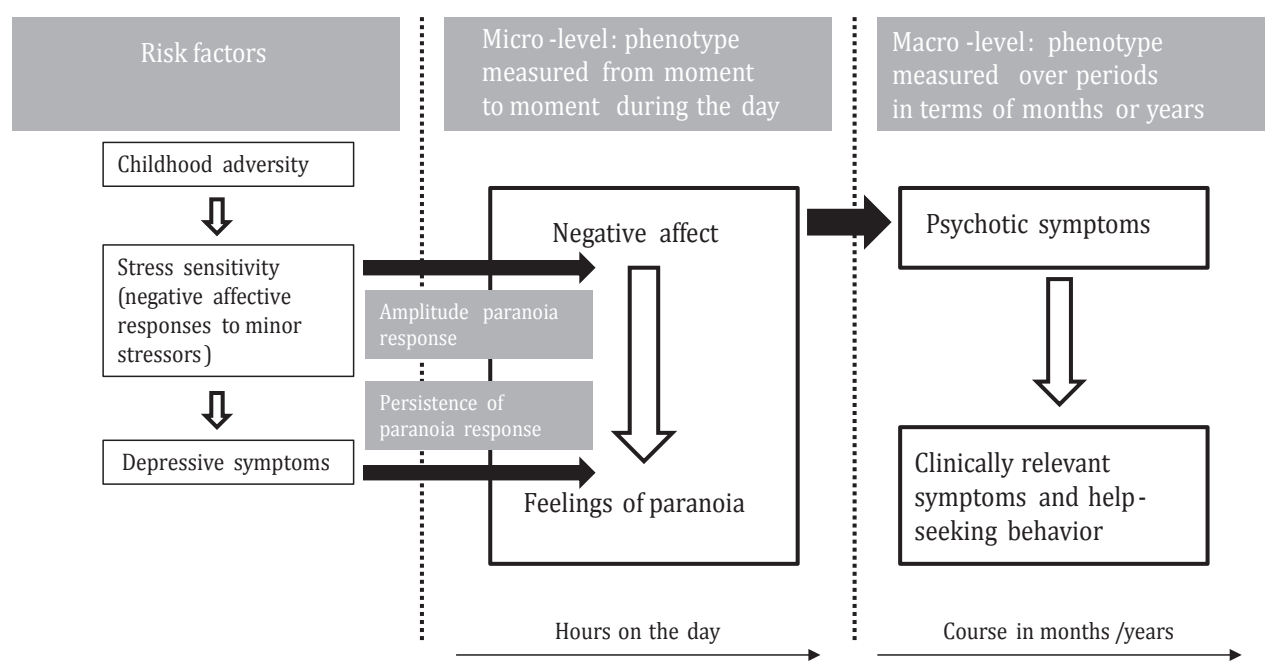

Figure 3 | The figure shows schematically how and why risk factors for psychosis may exert their influence on later macro-level course of psychotic symptoms by their impact on micro-level hour-to-hour emotional dynamics. Risk factors change the micro-level hour-to-hour interplay between negative affect (NA) and paranoia by increasing the amplitude or persistence of paranoia experiences following NA boosts. These subtle changes in repetitive everyday emotional dynamics are found associated with follow-up course of psychotic symptoms measured over periods of months. These psychotic symptoms, when persistent, may eventually result in clinical psychosis and help-seeking behavior.

(36), may be relevant in this context. For example, emotion regulation therapies directed at teaching reappraisal may decrease the types of negative experiences that give rise to paranoia and psychosis $(33,35,36)$. Future studies are needed to examine how potentially predictive real-life patterns of paranoia can assist in improving personalized risk assessment and clinical decision making (38).

\section{Methodological issues}

A critical remark is that effect sizes in this study are relatively small (under 0.2)(39). However, unlike effects reported in most unilevel studies, these effects do not impact once, but impact repeatedly on daily life experience. Therefore, effect sizes reported in the current study, although below Cohen's (39) indication of 0.2 , cumulatively may well be clinically significant.

We do not exclude the possibility that continuation of NA itself also induced continuation of paranoia over time. Thus, paranoia persistence may not be independent of co-occurring NA. However, in our opinion, this does not diminish the clinical relevance of the findings. Instead, it shows how closely NA is involved in development 
of subtle experiences of paranoia, which eventually and cumulatively may contribute to development of psychotic symptomatology.

Second, childhood adversity was measured retrospectively with a self-report questionnaire, which was adapted on request of the Flemish Twin Register. The total average childhood adversity score may be somewhat lower due to the omission of the four most explicit items. This may have resulted in reduced effect sizes of early adversity on paranoia following the NA increase. Also, twins may differ from singletons in that they may experience more social support (compared to non-twins) that buffers against psychopathology. If this is the case, then the current results likely reflect a conservative estimation of the effects in the general population. The low average childhood adversity score and its limited variability may also indicate that the subjects sample is a privileged and relatively healthy group.

Furthermore, ESM measurements of affect, stress sensitivity and paranoia are based on subjective reports. However, the prospective nature of the study and in-themoment collection of observations minimalized influences of recall bias or cognitive reinterpretation. Also, self-reported compliance, as assessed electronically in a subsample, was very high (96.4\%)(40). However, the use of an electronic ESM device in which the exact time of answering the questions is recorded will enhance accuracy of measurements.

Finally, subjects were female with a high mean educational level. Due to genderdifferences in risk factors for different pathways to psychosis (15), the results of the study may not be generalized to men and those with lower educational level.

\section{Acknowledgements}

This research was supported by the Netherlands Organisation for Scientific Research. Since its origin, the East Flanders Prospective Survey has been partly supported by grants from the Fund of Scientific Research, Flanders and Twins, a non-profit Association for Scientific Research in Multiple Births (Belgium). Dr. M. Wichers was supported by the Netherlands Organisation for Scientific Research (VENI grant nr. 916.76.147) and by ZON-MW (grant nr. 171001002). Prof. dr. I. Myin-Germeys was supported by a 006NARSAD Young Investigator Award and by the Netherlands Organisation for Scientific Research (VIDI grant nr. 917.76.341).

\section{Declaration of interest:}

The authors have declared that there are no conflicts of interest in relation to the subject of this study. 


\section{References}

1. van Os J, Linscott RJ, Myin-Germeys I, Delespaul P, Krabbendam L. A systematic review and meta-analysis of the psychosis continuum: evidence for a psychosis proneness-persistence-impairment model of psychotic disorder. Psychol Med. 2009;39(2):179-95.

2. Wigman JTW, van Winkel R, Raaijmakers QAW, Ormel J, Verhulst FC, Reijneveld SA, et al. Evidence for a persistent, environment-dependent and deteriorating subtype of subclinical psychotic experiences: a 6-year longitudinal general population study. Psychol Med. 2011;41(11):2317-29.

3. Buckley PF, Miller BJ, Lehrer DS, Castle DJ. Psychiatric comorbidities and schizophrenia. Schizophr Bull. 2009;35(2):383-402.

4. van Rossum I, Dominguez MD, Lieb R, Wittchen HU, van Os J. Affective dysregulation and reality distortion: a 10-year prospective study of their association and clinical relevance. Schizophr Bull. 2009;37:561-71.

5. Wigman JTW, van Nierop M, Vollebergh WAM, Lieb R, Beesdo-Baum K, Wittchen H-U, et al. Evidence that psychotic symptoms are prevalent in disorders of anxiety and depression, impacting on illness onset, risk, and severity-implications for diagnosis and ultra-high risk research. Schizophr Bull. 2012;38(2):247-57.

6. Wichers M, Schrijvers D, Geschwind N, Jacobs N, Myin-Germeys I, Thiery E, et al. Mechanisms of geneenvironment interactions in depression: evidence that genes potentiate multiple sources of adversity. Psychol Med. 2009;39(7):1077-86.

7. Varese F, Smeets F, Drukker M, Lieverse R, Lataster T, Viechtbauer W, et al. Childhood adversities increase the risk of psychosis: a meta-analysis of patient-control, prospective- and cross-sectional cohort studies. Schizophr Bull. 2012;38 (4):661-71.

8. Freeman D, Fowler D. Routes to psychotic symptoms: trauma, anxiety and psychosis-like experiences. Psychiatry Res. 2009;169(2):107-12.

9. Corcoran CM, Kimhy D, Parrilla-Escobar MA, Cressman VL, Stanford AD, Thompson J, et al. The relationship of social function to depressive and negative symptoms in individuals at clinical high risk for psychosis. Psychol Med. 2011;41(2):251-61.

10. van Rijn S, Schothorst P, Wout Mvt, Sprong M, Ziermans T, van Engeland H, et al. Affective dysfunctions in adolescents at risk for psychosis: emotion awareness and social functioning. Psychiatry Res. 2011;187(1):100-5.

11. Selten JP, Cantor-Graae E. Social defeat: risk factor for schizophrenia? Br J Psychiatry. 2005;187(2):101-2.

12. Wichers M, Geschwind N, Jacobs N, Kenis G, Peeters F, Derom C, et al. Transition from stress sensitivity to a depressive state: longitudinal twin study. Br J Psychiatry. 2009;195(6):498-503.

13. Wichers M, Myin Germeys I, Jacobs N, Peeters F, Kenis G, Derom C, et al. Genetic risk of depression and stress-induced negative affect in daily life. Br J Psychiatry. 2007;191:218-23.

14. Myin Germeys I, van Os J, Schwartz JE, Stone AA, Delespaul PA. Emotional reactivity to daily life stress in psychosis. Arch Gen Psychiatry. 2001;58(12):1137-44.

15. Myin-Germeys I, van Os J. Stress-reactivity in psychosis: evidence for an affective pathway to psychosis. Clin Psychol Rev. 2007;27(4):409-24.

16. Kramer IMA, Simons CJP, Myin-Germeys I, Jacobs N, Derom C, Thiery E, et al. Evidence that genes for depression impact on the pathway from trauma to psychotic-like symptoms by occasioning emotional dysregulation. Psychol Med. 2012;42(2):283-94.

17. van Os J, Linscott RJ. Introduction: the extended psychosis phenotype-relationship with schizophrenia and with ultrahigh risk status for psychosis. Schizophr Bull. 2012;38(2):227-30.

18. Delespaul P. Assessing schizophrenia in daily life: the experience sampling method. Maastricht, the Netherlands: Maastricht University Press; 1995.

19. Csikszentmihalyi M, Larson R. Validity and reliability of the experience-sampling method. J Nerv Ment Dis. 1987;175(9):526-36.

20. Thewissen V, Bentall RP, Oorschot M, à Campo J, van Lierop T, van Os J, et al. Emotions, self-esteem, and paranoid episodes: an experience sampling study. Br J Clin Psychol. 2011;50(2):178-95.

21. Jacobs N, Myin-Germeys I, Derom C, Vlietinck R, van Os J. Deconstructing the familiality of the emotive component of psychotic experiences in the general population. Acta Psychiatr Scand. 2005;112(5):394-401. 
22. Loos R, Derom C, Vlietinck R, Derom R. The East Flanders Prospective Twin Survey (Belgium): a population-based register. Twin Res. 1998;1(4):167-75.

23. Derom CA, Vlietinck RF, Thiery EW, Leroy FO, Fryns JP, Derom RM. The East Flanders Prospective Twin Survey (EFPTS). Twin Res Hum Genet. 2006;9(6):733-8.

24. Kendler KS, Thornton LM, Prescott CA. Gender differences in the rates of exposure to stressful life events and sensitivity to their depressogenic effects. Am J Psychiatry. 2001;158(4):587-93.

25. Simons CJP, Wichers M, Derom C, Thiery E, Myin-Germeys I, Krabbendam L, et al. Subtle gene-environment interactions driving paranoia in daily life. Genes Brain Behav. 2009;8(1):5-12.

26. Arntz A, Wessel I. Jeugd Trauma Vragenlijst [Dutch version of the Childhood Trauma Questionnaire]. Maastricht, the Netherlands: Author; 1996.

27. Bernstein DP, Stein JA, Newcomb MD, Walker E, Pogge D, Ahluvalia T, et al. Development and validation of a brief screening version of the Childhood Trauma Questionnaire. Child Abuse Negl. 2003;27(2):169-90.

28. Arrindel W, Ettema J. SCL-90. Een multidimensionele psychopathologie indicator [The SCL90. A multidimensional instrument for the assessment of psychopathology]. Lisse: the Netherlands: Swets \& Zeitlinger; 1986.

29. Derogatis LR. SCL90: administration, scoring and procedures manual-1 for the revised edition. Baltimore, MD: John Hopkins School of Medicine; 1977.

30. Stefanis NC, Hanssen M, Smirnis NK, Avramopoulos DA, Evdokimidis IK, Stefanis CN, et al. Evidence that three dimensions of psychosis have a distribution in the general population. Psychol Med. 2002;32(2):347-58.

31. StataCorp. Stata Statistical Software: Release 11. College Station, TX, USA: Statacorp LP; 2009.

32. Gross JJ. The emerging field of emotion regulation: an integrative review. Rev Gen Psychol. 1998;2(3):27199.

33. Gross JJ. Emotion regulation: affective, cognitive, and social consequences. Psychophysiology. 2002;39(3):281-91.

34. Kimhy D, Vakhrusheva J, Jobson-Ahmed L, Tarrier N, Malaspina D, Gross JJ. Emotion awareness and regulation in individuals with schizophrenia: implications for social functioning. Psychiatry Res. 2012; 200(2):193-202.

35. Gross JJ, John OP. Individual differences in two emotion regulation processes: implications for affect, relationships, and well-being. J Pers Soc Psychol. 2003;85(2):348-62.

36. Barlow DH, Allen LB, Choate ML. Toward a unified treatment for emotional disorders. Behav Ther. 2004;35(2):205-30.

37. Collip D, Myin Germeys I, Van Os J. Does the concept of "sensitization" provide a plausible mechanism for the putative link between the environment and schizophrenia? Schizophr Bull. 2008;34(2):220-5.

38. Wichers M, Simons CJP, Kramer IMA, Hartmann JA, Lothmann C, Myin-Germeys I, et al. Momentary assessment technology as a tool to help patients with depression help themselves. Acta Psychiatr Scand. 2012;124(4):262-72.

39. Cohen J. Statistical power analysis for the behavioral sciences. Hillsdale, NJ: Lawrence Earlbaum Associates; 1988.

40. Jacobs N, Nicolson NA, Derom C, Delespaul P, van Os J, Myin-Germeys I. Electronic monitoring of salivary cortisol sampling compliance in daily life. Life Sci. 2005;76(21):2431-43. 



\section{PART 2}

Experience Sampling Method for treating psychopathology 


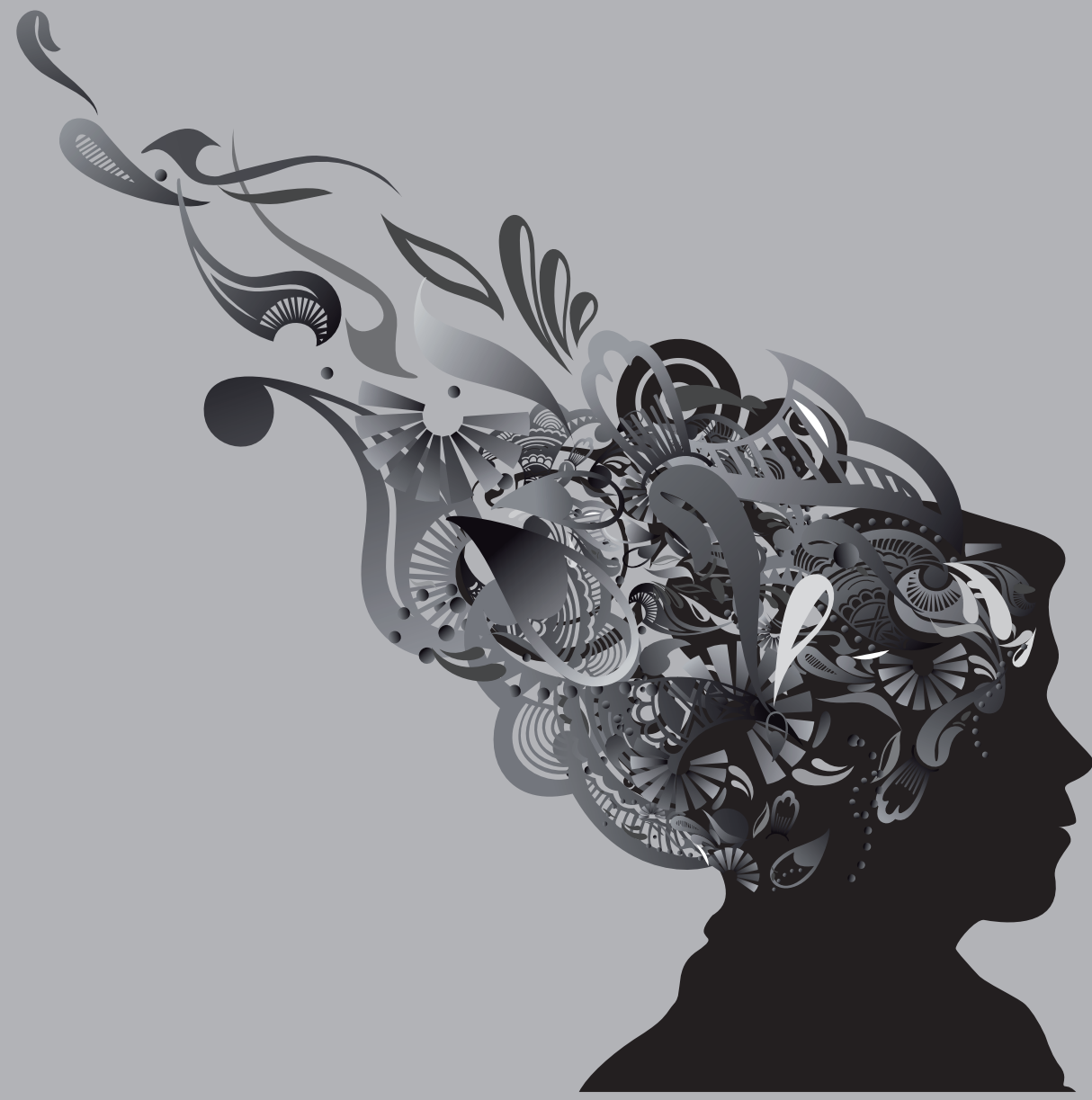




\section{CHAPTER 4}

\section{Momentary assessment technology as a tool to help patients with depression}

\section{help themselves}

M. Wichers ${ }^{1}$, CJP. Simons ${ }^{1,2^{*}}$, IMA. Kramer ${ }^{1,2^{*}}$, JA. Hartmann ${ }^{1,2}$, C. Lothmann ${ }^{1}$, I. Myin-Germeys ${ }^{1}$, AL. van Bemmel ${ }^{2}$, F. Peeters ${ }^{1}$, Ph. Delespaul ${ }^{1}$, J. van Os ${ }^{1,3}$

${ }^{1}$ Department of Psychiatry and Psychology, South Limburg Mental Health Research and Teaching Network, EURON, Maastricht University, the Netherlands

${ }^{2}$ GGzE, Institute of Mental Health Care Eindhoven and the Kempen, Eindhoven, the Netherlands ${ }^{3}$ King's College London, Department of Psychosis Studies, Institute of Psychiatry, London, UK * Contributed equally 


\section{Abstract}

Objective: Given high relapse rates and residual symptoms in depression, new strategies to increase treatment effectiveness are required. A promising avenue is to investigate how electronic momentary assessment technology may contribute to clinical assessment and interventions in depression.

Method: A literature search was conducted focusing on the potential contribution of momentary assessments to clinical applications in depression.

Results: Momentary assessments are able to reveal subtle, small but repetitive and relevant patterns of emotional expression that predict future course of depression. A momentary assessment tool may expose manageable pieces of daily life behavior contributing to the depressive experience that patients can influence. The use of this explicit knowledge of daily life experience is understudied with regard to its contribution to diagnostic assessment, monitoring of treatment effects and feedback interventions in depressed patients. The clinical application of momentary assessments may stimulate a shift from passive consumption of treatment to an active role for patients in their recovery and increased patient ownership.

Conclusion: The precise, prospective and fine-grained information that momentary assessment technology provides may contribute to clinical practice in various ways. Future studies should examine the clinical impact of its use and the feasibility of its implementation in mental health care.

\section{Clinical recommendations:}

- The fine-grained nature of momentary assessment data allow for precise and prospective monitoring of the effects that antidepressants have on a patient's experience.

- Translating the 'film of daily life' into person-tailored feedback may expose manageable pieces of daily life behavior contributing to the depressive experience that patients can influence.

- The active role for patients in clinical applications of momentary assessment technology may reduce stigma and increase feelings of control, ownership, and compliance with medication. 
Additional comments:

- Long-term monitoring of mood states using momentary assessments may be demanding for depressed patients. Feasibility and tolerability need to be examined in more detail.

- A momentary assessment tool is proposed as complementary to care-as-usual, and not to replace face-to-face contact with general practitioners or mental health care professionals.

- Thus far, ambulatory monitoring in depressed patients has been carried out only in the context of research. The feasibility of the implementation in mental health care needs to be established. 


\section{Introduction}

In terms of ranking by person-years reduction in quality of life (1), major depressive disorder (MDD) is consistently among the group of disorders with the most impact in the world population. As a result, societal costs because of health care consumption and decreased work productivity are high (2). Although psychotherapeutical and psychopharmacological treatments are available for the treatment of MDD, the vast majority of patients is being treated with antidepressant medication (ADM) in primary and secondary care settings (3). The efficacy of ADM has been established in many randomized controlled trials (RCTs), but translation to effectiveness in daily practice remains highly problematic. Despite an increasing number of psychopharmacological treatment options $(4,5)$, most patients do not take their medication as prescribed $(6)$, and among those who do, about one-third remain treatment-resistant (7-9). In addition, it has been shown that antidepressants, compared to placebo, only work in patients at the highest end of the severity spectrum, and are not effective for people with mild and moderate levels of depression (10). This is problematic given that low and moderate levels of depression are also associated with higher levels of morbidity and health care consumption at the population level (11). Combining medication with psychotherapy may increase the effectiveness of the treatment (12). However, psychotherapy is not routinely available, and relapse rates following treatment remain high (around 30\%) (13). Furthermore, systematic reviews show that psychotherapy, similar to ADM, may be less effective than previously thought (14).

Given these considerations, it may be productive for research in the area of depression and its treatment to resort more to "out of the box" thinking to develop new strategies for clinical practice, focussing on improving efficacy as well as effectiveness through enhanced patient engagement. Taking medication provides neither incentive nor obligation to mobilize individual resources. Patients who passively receive antidepressants for a medical diagnosis of depression are not made active participants in either the diagnostic or the therapeutic process. In practice, passive receipt of diagnosis and therapy may contribute to the experience of disempowerment, reducing the likelihood of active engagement in the process of recovery (15).

Electronic momentary assessment techniques allow for precise and prospective monitoring of emotions experienced in a daily life context. These could provide unique person-tailored insights to both clinicians and patients about the nature, context and (intervention-related) changes in emotional patterns in daily life and, thereby, new possibilities for assessment and treatment in depression. 


\section{Aims of the study}

This article is set up not with the aim of providing a full literature review on the topic, but rather to discuss why and how electronic momentary assessment tools may contribute to clinical assessment and treatment in depression.

\section{Material and methods}

A literature search was conducted focusing on the possibilities of momentary assessment techniques with special reference to how these data may bring about insight into the depressive symptomatology both for the patient and the treating clinician and how momentary assessments could be of use in revealing emotional and behavioral patterns relevant to clinical assessment and treatment in depression. The results section will start with an overview of how several methods of self-monitoring have already been applied in clinical practice both within and outside the field of psychiatry. The second part will focus specifically on the possibilities of momentary assessment techniques as currently applied in research. The final part discusses how momentary assessment techniques could be used in clinical applications for the assessment and treatment of depression.

\section{Results}

\section{Translating the black box of 'depression' to quantifiable patterns of emotional experience}

Depression is a complex disorder characterized by strong dysregulation of emotional experience. Tools like momentary assessment techniques are available and used in research to identify patterns of moment-to-moment experiences $(16,17)$. Surprisingly, however, knowledge about in-the-moment patterns of daily life emotional experience in depressed patients is not available for clinicians to aid treatment or decisions on diagnosis in traditional settings. Although the implicit assumption is that in-the-moment daily life patterns of emotional experience underlie the symptoms and will improve with treatment, it is never made explicit. Therefore, there is a need to make the implicit explicit. A translation from implicit, moment-to-moment emotional reactivity to explicit, visualized and quantifiable patterns of emotional experience is required in order for patients and clinicians alike to understand, modify and track the experiences that are currently subsumed under the 'black box' diagnosis of depression. The 'tracking' of moment-to-moment experiences in the flow of daily life that enables visualization and quantification of affective patterns and responses in daily life is very different from other methods currently in use in treatment settings. For example, life chart methodology 


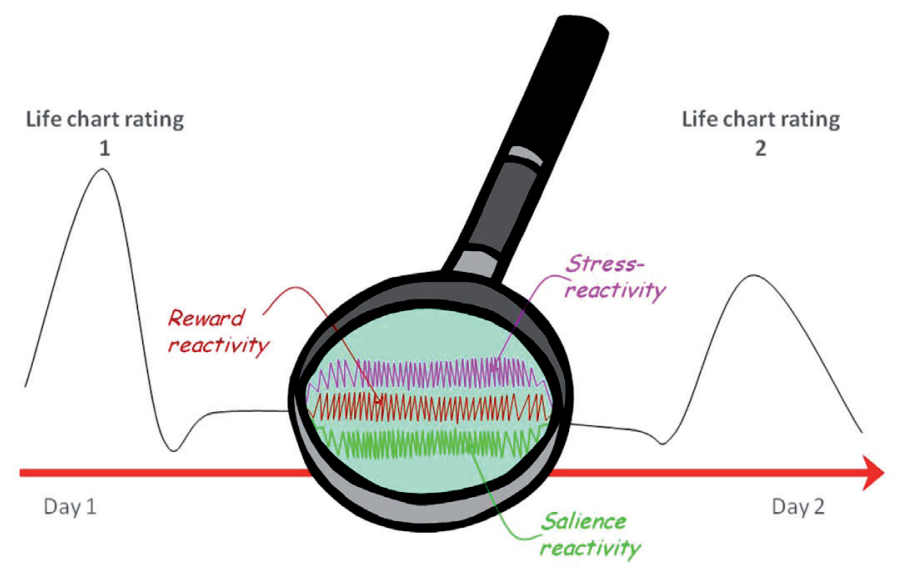

Figure 1 | Life charts are based on global judgements of mood over the entire day, while momentary assessment technology is able to zoom in on the precise moment-to-moment dynamic patterns of emotions over the day, and how they relate to daily life situations and contexts. For example, hour-to-hour processing of interactive person-context patterns such as reward reactivity (positive affective responses to positive events), stress reactivity (negative affective responses to events), salience reactivity (attribution to salience events) and many other relevant patterns can be obtained using momentary assessments.

$(18,19)$, regularly used in patients with bipolar disorder, outlines past and current course of illness in the form of a graph that visualizes manic and depressive episodes, hospitalizations and intake of medication. Retrospective life chart ratings are recorded by month and year, while prospective ratings are performed on a daily basis where patients can differentiate between four levels of severity to describe their experience. Unlike momentary assessments, made in the moment, life chart methodology describes experiences retrospectively. Even 'prospective' life chart ratings of mood states involve ratings based on retrospective recall and global judgement of mood over an entire day. A single rating of mood per day gives no information on moment-to-moment dynamic patterns of emotions over the day, how they relate to daily life situations and contexts, and how they fluctuate and change in response to certain situations (Figure 1). Thus, momentary assessment methodology $(20,21)$ would be needed to make the implicit, in-the-moment dimension of emotional life that is dysregulated in depression, explicit to both patients and clinicians.

\section{The use of self-monitoring}

Symptom monitoring in the area of somatic medicine is a well-established tool to collect information on individual risk and guide treatment decisions. Research has shown, for example, that 24-h monitoring of blood pressure or plasma glucose levels yields much 
better indicators of illness and risk than a single assessment in the doctor's office (2224). It better represents a patient's daily life functioning and can reveal, for example, how blood pressure responds to daily life stress or sleep $(22,23)$. In the treatment of diabetes, continuous glucose monitoring has been shown to help patients see patterns and the impact of self-management behaviors (24). Ambulatory technology designed for continuous assessment of motor symptoms has also been used in the rehabilitation of patients with Parkinson's disease. The extra value above treatment as usual is described as 'the window of observation' in the home of the patients that becomes available for medical professionals $(25,26)$. Thus, with the advent of many technological innovations, tools for continuous monitoring have become increasingly available outside the doctor's office. Patients now can auto-monitor heart rate or blood pressure in the flow of daily life, and take preventive actions by changing daily routines designed to reduce risk of illness, relapse or progression. Surprisingly, although systematic daily life monitoring now is widely available for many physiological outcomes, it remains to be extrapolated to the therapeutic context of psychiatric disorders such as MDD.

Active self-monitoring of emotional experience in psychiatry is the next obvious step, analogous to the development of technology for this purpose in somatic medicine, as there are strong a priori reasons to hypothesize that it will complement current clinical practice in an effective way. Self-monitoring requires active patient engagement in the process of recovery and may give patients insight into the nature and dynamic fluctuations of their depressive symptomatology, and the link between symptoms and daily life emotional patterns of reactivity. These insights may engender feelings of control and empowerment in relation to symptoms (27). Recently, a case-study was published in which a mental health care user with a diagnosis of depression described his struggle with the illness and the way he coped with symptoms. The patient, a researcher with a $\mathrm{PhD}$ in genetics, started to prospectively assess his moods by scoring them daily on a scale of 1 to 10 on the basis of self-selected criteria. He thus constructed a mood journal depicting his own mood swings across a period of 5 years, describing the effect of self-monitoring as follows:

'I have now been diagnosing my mood swings every day for 5 years. This has been very helpful: now I look at my own mood swings in a different way. I can accept more easily the gloomy periods I still have once in a while.' 'Even though I had learned over time that my mood could change suddenly, I had never been able to get a handle on them. This became possible by reviewing these graphs. It was suddenly clear what I had never been able to come to grips with: the unavoidability of these constantly recurring gloomy periods. They display a regularity of recurrence, but also, and at least equally important, it may be observed that they always, and equally without clear reason, go away. This did 
not make me hopeless or powerless, but, paradoxically enough, gave me confidence'... 'The 'guilt' for these gloomy moods came to be located outside myself.' 'Their threat is also much lower than before and it is now easier to accept them without needing to find a cause for them. Just realizing this gives me the motivation, even when in the throws of these moods, to get something done, when before my only response was to withdraw into myself.' 'It made me 'see' my problem in the face, understand it, take perspective on it from a distance'.

This $n=1$ study suggests that the principle of continuous self-assessment may aid acceptance of depression and that patients can be empowered to diagnose experiences as a means to understand the sources and consequences of continuous mood variation over time (27). Below, we will demonstrate how these principles may be extended to the much more revealing and productive practice of self-monitoring of momentary emotional states and reactivity in the flow of daily life, also anticipated by the user in the $n=1$ study above (27).

\section{Momentary assessment methodology}

The monitoring system cited above consisted of the recording of one measurement each day on a mood scale ranging from 1 to 10 . Such a simple monitoring system is frequently used in clinical psychiatry; an example is the life chart for patients with bipolar disorder $(18,28)$, which research suggests is useful $(18)$. However, a much more sophisticated, prospective self-monitoring application is also available, which to date has been used exclusively at the level of research. This application is called 'the experience sampling method (ESM)' or 'ecological momentary assessment (EMA)' $(20,21,29)$. These names refer to research tools that are used to conduct frequent and prospective momentary assessments of experiences of emotions, activities, events and other contextual variables in the realm of daily life. Both random and fixed assessment time points can be used depending on the study hypothesis. These momentary assessment techniques allow for measurements not just once a day, but also -for example- at random moments in intervals of on average $90 \mathrm{~min}$ apart from each other. They allow for a more distinctive and specific assessment of mood by using assessments of various types of affect ('At this moment I feel .... enthusiastic, content, gloomy, irritated, anxious, cheerful, paranoid, relaxed, sad etc.'). In addition, momentary assessment technology not only provides for an assessment of mood in the moment, but also assesses "embeddedness" of experiences by rating appraisals of current company, activities, minor events and other items about the context in which mood (changes) are situated. As described above, it is this method that is able to track moment-to-moment experiences and to make implicit mood dysregulation explicit in quantifiable patterns of fluctuation and reactivity of emotional experience in daily life. 


\section{Methodological and statistical strengths of momentary assessment methodology}

In mental health research, momentary assessment methodology is gaining popularity (30-33) as it has several important advantages over gathering information retrospectively. The fact that it offers real-time assessment is important in the field of affective disorders, because the recall of emotions is influenced by several psychological processes that may bias and distort memory. An example is the mood congruency effect, i.e. enhanced recall of positively valenced memories when in a positive mood state and, vice versa, enhanced recall of negatively valenced memories when experiencing a negative mood state $(34,35)$. Also, duration of an experience, when assessed retrospectively, tends to be underestimated (36). Studies assessing both momentary and retrospective ratings of emotions (37-40) have shown that there is only moderate agreement between the momentary and the recalled experience of emotions and that retrospective recall patterns were characterized by under- and overestimation of emotions (overestimation of negative emotions in patients with borderline personality disorder and major depression and of positive emotions in healthy controls).

Furthermore, momentary assessment methodology includes multiple repeated (within-subject) measurements in the flow of daily life. The use of within-subject measurements has been advocated previously by several research groups $(41,42)$. While most studies examine between-subject differences at a single measurement occasion, within-subject data provide mental health research with the opportunity to further examine intraindividual changes in relation to daily life situations and experiences. Momentary assessments may be able to reveal subtle, small but repetitive and relevant patterns of emotional expression that are not picked up by depression questionnaires or even interviews (43-45). To have the 'film' rather than a 'snapshot' of daily life may uncover important mechanisms and processes involved in affective disorders. Depression research has just started to use momentary assessments to answer questions of how the 'film' of daily life dynamic processes of affective characteristics may relate to risk for, and resilience against, depression. Affective characteristics that have been examined, for example, are intraindividual variations in affect or affective responses to the daily life context $(16,17$, $43,46-55)$, but also the impact of intraindividual changes in positive emotions on people's negative emotions in the next few hours and vice versa (56). Other questions recently tackled involve (the change in) affective characteristics related to ADM and response to treatment (57-60). These dynamic emotional processes that take place within individuals every day and interact with the person's context or with other emotions over time not only appeared strongly associated with risk and resilience towards depression $(61,62)$, but also with response to treatment. Various observed subtle daily life patterns of affect - such as the prospectively measured dynamic interplay between negative and positive emotions, the extent to which positive emotions are retained over time (in hours), low 
levels of fluctuations of negative emotions and the ability to generate positive emotions from small pleasant events - are associated with the prevention of future depressive relapse and with future successful recovery from depression $(43,52,56-58)$.

\section{Clinical application of momentary assessment methodology}

Given the fact that i) new strategies have to be examined that may improve therapeutic efficacy/effectiveness in depression, ii) that both results of scientific research and experience of patients point towards the value of the monitoring of emotional responses and iii) that the advent of technology to track emotions continuously and in real time without the use of paper and pen, the time has come to examine in what ways momentary assessment-based technology can be of use in current clinical practice.

Recent software packages have been developed that allow these research protocols to run on electronic devices $(31,63)$. This development enables new mobile intervention strategies (63-65). However, of all recently developed mobile interventions, only some provide person-tailored feedback instead of standard messages $(64,65)$, and only very few use person-tailored feedback in combination with momentary assessment technology (65-67). In these few studies, momentary assessments have been used for fine-grained measures of momentary experiences to identify moments at which patients might benefit from a (non-person-tailored) reminder of certain self-management strategies. An undeveloped area so far is the use of the richness of the momentary assessment data in person-tailored feedback. The strengths of momentary assessment methodology in research that have recently uncovered some relevant daily life patterns of experience in relation to depressive symptomatology $(43,52,57,58,61,62)$ need clinical translation in the form of person-tailored feedback. Momentary assessment methodology allows for feedback on dynamic patterns of emotions, the associations between emotions and certain daily life contexts and changes in these parameters over time. Another underdeveloped area in research is the use of momentary assessments to aid diagnostics and assessment of treatment effects. Possibilities and relevance of the use of momentary assessment methodology in these areas is discussed below.

The introduction of momentary assessment methodology in clinical practice depends on the development of new digital instruments programmed for the collection of real-time data. Furthermore, the use of momentary assessment information requires instruments that enable direct access to the collected data for feedback to patients and clinicians. One newly developed instrument that fulfils these criteria is the 'PsyMate' (Figure 2). The PsyMate was purpose-made, to facilitate the monitoring of daily life experience and behavior. It can be programmed to generate beeps at unpredictable moments of the day, and participants use a touch screen to fill out small questionnaires on current affective state, social context and activities in response to a beep signal. 


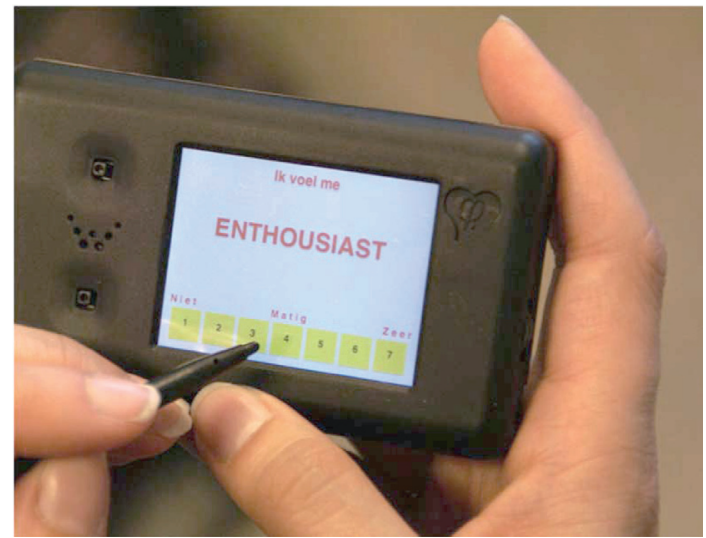

Figure 2 | PsyMate device

Furthermore, it is small, can store all data and additionally can be programmed to become interactive (i.e. respond to the input of the subject). Other digital devices, such as personal digital assistants (PDAs) programmed for momentary assessment protocols, may also be equipped for this purpose. Researchers should consider whether the protocol is better served by devices that give participants the option to switch off power and/or alter sound volume. An advantage of such flexibility is that level of engagement and research adherence is in the hands of participants. A disadvantage is that adherence may suffer as a result of moments where participants unintentionally forget to switch power and/or volume back on. Also, for an eventual widespread use in clinical practice, a device should be relatively inexpensive. Although currently expensive, digital devices such as PDA's and smart phones may become attractive as costs of these devices decrease. Digital data collection is suitable for long-term monitoring, as it requires less subject time investment than the paper-and-pencil method. Below, several applications are described through which the continuous mood tracking facilities of momentary assessment instruments may be put to use in therapeutic practice.

\section{Momentary assessment technology as a tool to diagnose and assess treatment effects}

Because ambulatory monitoring in other fields of medicine has shown to improve diagnostic assessment and therapeutic practice $(22,23,25)$ and retrospective recall of prior mood states is highly unreliable (37), patients and clinicians in mental health services might benefit from a monitoring system that provides prospective, daily life and context-related information on changes in mood and experience. The availability of relevant daily life characteristics such as affective responses to a certain context (e.g. 
social context, activities or events) may give the clinician a better grip on the nature of the depression at hand, not only in terms of depth and persistence of low mood, or the balance between negative and positive emotions $(68,69)$, but also of prospective daily life patterns of, for example, levels of rumination or sleeping habits as available in data collected with the PsyMate or other digital devices.

Daily life mood tracking can also be used for long-term monitoring of changes in affective patterns. Unlike other branches of medicine, mental health care has been slow to develop systems to monitor medication effects. Digital momentary assessment devices (hereafter: "MA devices") may be used to follow patients from the start of antidepressant - or any other- medication in order to give patients and clinicians increased insight in how medication impacts on daily life mood states. The explicit feedback on momentary patterns of affect and appraisals of daily life situations may help patients to see more easily whether there are subtle positive changes over time that they might not have been conscious of without feedback. These momentary patterns are likely more sensitive than global retrospective (self-) assessments in signaling the earliest signs of improvement. It may also help clinicians decide whether continuation or change of medication type or dose is the best way forward and reduce unnecessary prescription of ineffective medication. Furthermore, the use of MA devices allows for the prospective monitoring of side effects of ADM.

Also, an important potential shift in patient ownership may arise through use of the MA devices. Assessment of the impact of pharmacological treatment in general practice or mental health services is often based on sketchy information as time is limited, and retrospective recall of mood states and changes therein is unreliable. In addition, the decision to continue using antidepressants or not is very much presented as an expert decision based on higher knowledge not available to the patient, thus reducing ownership and motivation to comply. Making the patient the expert/owner of the information on the basis of which is decided whether or not the treatment has had any effect may remedy this. The MA device may represent a tool to collect information on the basis of which a diagnosis is made jointly by patient and clinician -both from their own perspective of expertise- before the prescription of any treatment. Furthermore, feedback of these data to patients may provide immediate positive reinforcement on early clinical improvement, which may impact positively on treatment compliance. Finally, patients may feel more inclined to comply with medication if they know effects are monitored and are taken into consideration.

\section{From implicit to explicit: momentary assessment technology as a tool for empirical, 'patient-owned' intervention}

The systematic collection of mood and experience will enhance awareness of the nature 
A
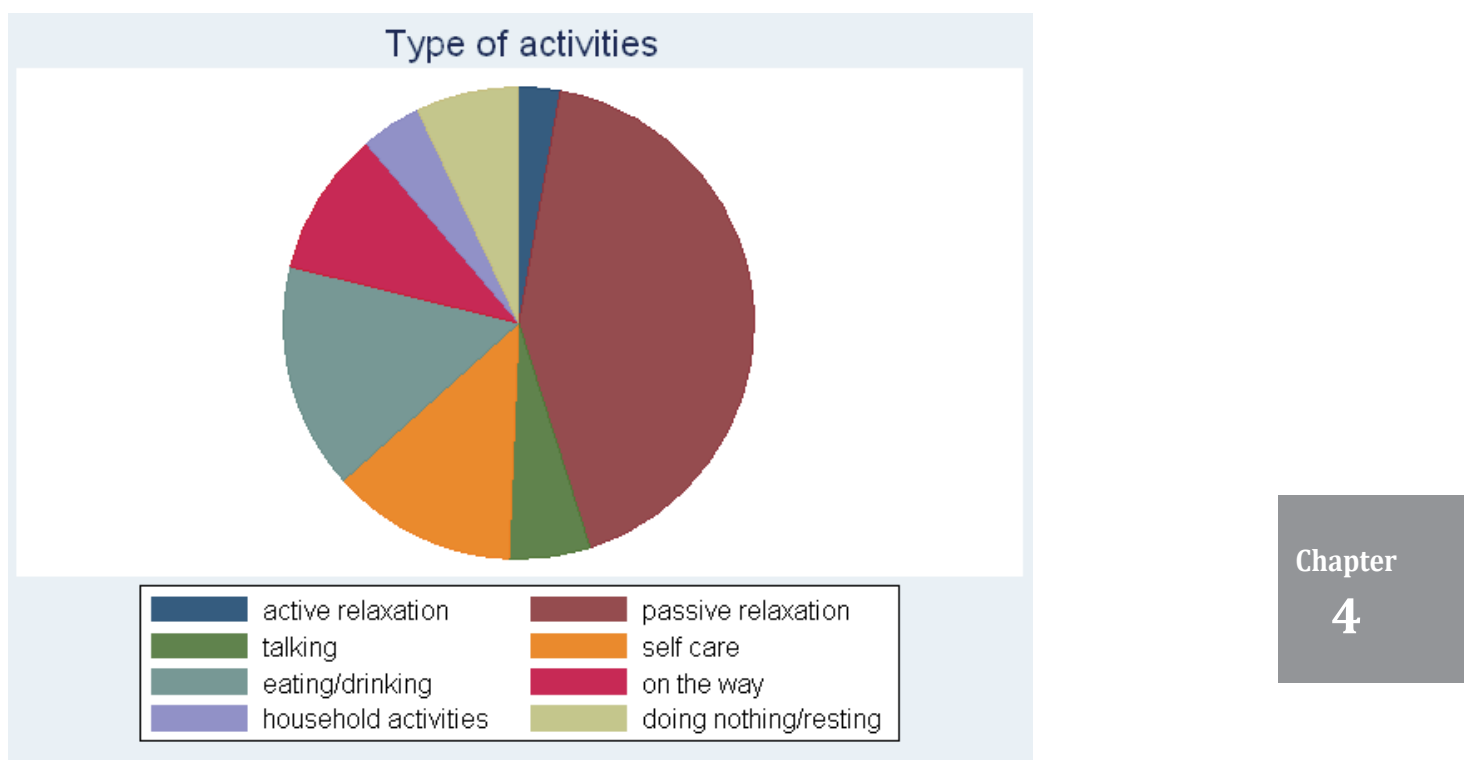

B

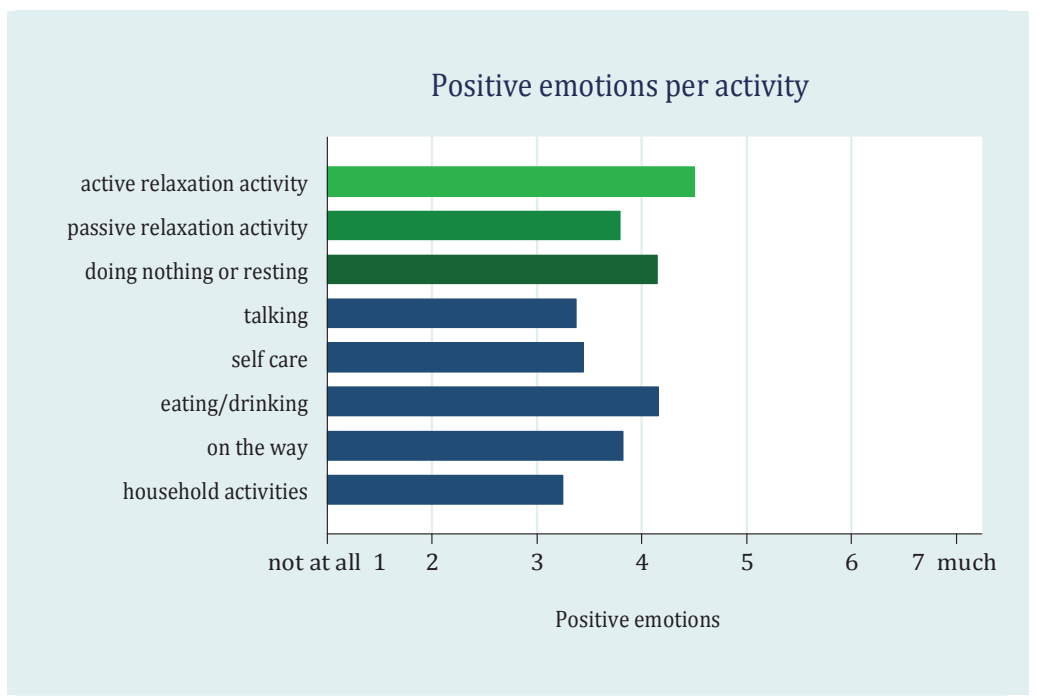

Figure 3 | (A) Pie graph on the time expenditure per type of activity of an individual. (B) Bar graph showing that engagement in active relaxation is associated with more positive emotions than engagement in passive relaxation for this individual. 
of symptoms, the patterning, changes over time and the contexts in which they occur. This will actively engage patients in the process of recovery and increase the patient's insight into the nature of the emotional dysregulation. Awareness of the coming and going of depressed mood states, their regularity and unpredictability, brought about by visual inspection of daily mood records (27), may facilitate insight into the need for change and increase feelings of confidence and control in patients. Some depressed individuals even commented following participation in a momentary assessment study designed for scientific reasons, not for feedback of momentary patterns to participantsthat responding to the questionnaires 'helped' them (40).

Therefore, explicit feedback of the data collected with MA devices may be used as a therapeutic intervention to provide patients with insight into functional and dysfunctional emotional responses. This will give patients the opportunity to reshape their environment in ways that are conducive to positive emotional experience. Ownership again is an important theme, as patients would be actively involved in their own empirical recovery process, based on making implicit, in-the-moment emotional processes explicit with the help of a reconstruction of emotional life based on momentary assessment data. Patients would not passively wait for someone or something (medication) to pull them back from the 'black box' of their depression diagnosis. Instead, they would be given an instrument to decompose the black box into manageable pieces of daily life experience that they can influence. A recent study used PsyMate-generated feedback in depressed patients as an intervention (70). Feedback included graphs showing people's time expenditure per type of daily life activity and their levels of positive emotions per activity. An example of such feedback is shown in Figure 3 for one depressed patient (the subject gave written approval for publication of the data). Figure 3a shows that this person tended to spend a substantial amount of time in passive leisure time relaxing activities and only very little time on active leisure time activities. The bar graph in Figure $3 b$ shows that this person experienced more positive emotions while engaging in active rather than passive relaxation. In response to such explicit feedback, patients may gain insight in implicit emotional patterns, allowing for targeted change of activities that may benefit his/her emotional well-being (in this case engaging more in active rather than passive relaxation). Similar feedback can be generated for positive emotions in social contexts, physical activity and other relevant behavior. This feedback may help patients to formulate concrete steps on how to change their daily life patterns in a way that aids recovery. In other words, momentary assessment technology may help patients to help themselves. Intrinsic learning processes owing to behavioral reinforcement may add the therapeutic process. Therefore, it might be hypothesized that the long-term effect of the intervention lasts longer than following passive consumption of antidepressants only. These are of course speculations that need to be further investigated. 
Momentary assessment technology interventions could be an attractive and inexpensive option to augment ADM, but other applications can also be envisaged. Unlike strategies based on reliance on patient recall of activities and mood state, or more crude diary methods, momentary assessment technology, with its prospective assessments, is able to monitor the more subtle emotional patterns and responses to situations over the day and is therefore a tool that may facilitate and augment some of the therapeutic goals of CBT (40). Finally, it is known that many people who experience depressed symptoms or a full-blown major depressive disorder do not seek treatment. This may be related in part to the threshold people experience in visiting a mental health professional and the need to overcome the stigma associated with that decision. There is evidence that a desire to manage the mood problem oneself was the most common reason for depressed people to avoid seeking treatment (71). In addition, psychotherapy is expensive and not routinely available or reimbursable in mental health services, forcing most to undergo the less expensive option of ADM or no treatment at all. Momentary assessment technology may provide an inexpensive and direct option for patients to first try to tackle their problems themselves. The first observations suggest that such an intervention is feasible and acceptable for patients (70) .

\section{Momentary assessment technology as a tool for prevention}

Given the chronicity of depression, the frequency with which it occurs and its invalidating nature, the psychological and economical costs are very high $(1,2)$. Residual depressive symptoms following remission of major depression are frequent and highly predictive of new episodes (72). In other words, depression is too expensive, at societal level, to invest only in cure; there is a pressing need to focus on prevention as well. New strategies are required for the long-term management of subthreshold and residual depressive symptoms. In particular, strategies regarding self-management of symptoms in remitted patients, for example self-management of emotional dysregulation using momentary assessment technology complementary to ADM, may be a realistic and cost-effective option to reduce residual symptoms and risk for recurrence. In addition, self-management of emotional dysregulation in vulnerable individuals may prevent future episodes of depression.

\section{Discussion}

An attempt was made to give an impression of how electronic momentary assessment tools may help clinical diagnosis and treatment in MDD. The main advantages of its use are that i) data on prospective and frequent assessments of daily life experiences can be provided to both clinicians and patients, which aids (self-)diagnosis and insight, 
ii) the information it provides on patterns of emotions and behaviors in daily life is person-tailored, iii) it provides patients with a tool to actively self-manage symptoms, which is likely to feed feelings of control and empowerment and iv) it may prove to be a low-cost treatment add-on that reduces the economical and psychological burden of MDD in society.

Some further remarks are called for. In our view, the use of a momentary assessment tool in clinical practice is not meant to replace the face-to-face contact with general practitioners or mental health care professionals. A technological tool allowing patients to take on an active role in their recovery process is not meant to augment current trends in society of individualization and devaluation of the need for interconnectedness with other people, as these processes more likely represent a contributing factor rather than the solution for common mental disorder in western societies. It is meant to complement traditional care in giving patients the best of both strategies and to provide an alternative for people who would otherwise not seek treatment, not receive psychotherapy or be on a waiting list for psychotherapy. It is meant to lower stigma and increase active involvement and empowerment of patients. Also, the tool may be used in vulnerable individuals to increase quality of life and emotional well-being, increasing resilience against depression.

Another issue is that the use of the MA devices may be demanding for patients with a full-blown depressive disorder, because the procedure is intensive and motivational impairment is a core characteristic of depression. The lack of motivation and energy in patients, therefore, is a potential threat to the success of MA devices for clinical purposes, especially in severe depression. Preliminary results of 21 depressed patients (70) suggested that the number of beeps per day to which patients have to respond is the most challenging aspect. Although the current protocol was challenging to some extent, most of the depressed patients in the experimental feedback condition indicated that if they would have had the choice they would have wanted to continue the intervention.

The suggested advantages of the use of momentary assessment technology in clinical practice remain speculative. Future studies need to examine what this technique can contribute to the care of depressed patients and to what extent it can improve diagnosis and treatment.

\section{Boosting patients' own recovery mechanism}

A recent meta-analysis (10) on the effect of antidepressants showed that these compounds show efficacy only in patients with more severe forms of MDD. Depressed people with low to moderate depression scores have similar rates of response and recovery when treated with either an active drug or placebo. The strength of the placebo response and a recent study $(n=2848)$ showing that treatment response seems 
to follow similar time courses in case of active or placebo compounds (73) suggests that mobilization of the patient's own capacity for recovery plays an important role. Therefore, boosting the patient's own capacity for recovery seems a promising new direction. Helping patients to help themselves, providing insight into and ownership of (changes in) daily life emotional patterns, thus actively engaging them in the process of recovery may be a first step to complement traditional care with a focus on the strengths and resilience mechanisms of individuals as owner of their experiences.

\section{Acknowledgements}

PsyMate development was made possible in part by unrestricted grants from Servier and Janssen-Cilag.

\section{Declaration of interest:}

PsyMate is developed under the auspices of the Maastricht University technology transfer office; none of the authors will benefit financially. Dr. F. Peeters received speakers' fees from GlaxoSmithKline, Wyeth, Astra Zeneca, Lundbeck, Eli Lilly, Servier, and Janssen-Cilag. Jim van Os is/has been an unrestricted research grant holder with or has received financial compensation as an independent symposium speaker from Eli Lilly, BMS, Lundbeck, Organon, Janssen-Cilag, GSK, AstraZeneca, Pfizer and Servier, companies that have an interest in the treatment of mental health disorders. Dr. Ph. Delespaul received speakers' fees from Lundbeck and Janssen-Cilag. Prof. Inez MyinGermeys received speaker-fees from Janssen-Cilag and Bristol-Myers Squibb. Dr M Wichers, Dr CJP Simons, IMA Kramer, JA Hartmann, C Lothmann and Dr AL van Bemmel had no financial interests. 


\section{References}

1. Mathers CD, Loncar D. Projections of global mortality and burden of disease from 2002 to 2030. PLoS Med. 2006;3(11):e442.

2. Donohue JM, Pincus HA. Reducing the societal burden of depression: a review of economic costs, quality of care and effects of treatment. Pharmacoeconomics. 2007;25(1):7-24.

3. van Marwijk HW, Bijl D, Ader HJ, de Haan M. Antidepressant prescription for depression in general practice in the Netherlands. Pharm World Sci. 2001;23(2):46-9.

4. Lam RW, Kennedy SH, Grigoriadis S, McIntyre RS, Milev R, Ramasubbu R, et al. Canadian Network for Mood and Anxiety Treatments (CANMAT) clinical guidelines for the management of major depressive disorder in adults. III. Pharmacotherapy. J Affect Disord. 2009;117 (Suppl 1):S26-43.

5. NICE. Depression: management of depression in primary and secondary care. Clinical Practice Guideline No. 23. London, UK: National Institute for Clinical Excellence; 2004.

6. Pampallona S, Bollini P, Tibaldi G, Kupelnick B, Munizza C. Patient adherence in the treatment of depression. Br J Psychiatry. 2002;180:104-9.

7. Little A. Treatment-resistant depression. Am Fam Physician. 2009;80(2):167-72.

8. Shelton RC, Osuntokun O, Heinloth AN, Corya SA. Therapeutic options for treatment-resistant depression. CNS Drugs. 2010;24(2):131-61.

9. Rush AJ, Warden D, Wisniewski SR, Fava M, Trivedi MH, Gaynes BN, et al. STAR*D: revising conventional wisdom. CNS Drugs. 2009;23(8):627-47.

10. Fournier JC, DeRubeis RJ, Hollon SD, Dimidjian S, Amsterdam JD, Shelton RC, et al. Antidepressant drug effects and depression severity: a patient-level meta-analysis. JAMA. 2010;303(1):47-53.

11. Judd LL, Akiskal HS, Zeller PJ, Paulus M, Leon AC, Maser JD, et al. Psychosocial disability during the longterm course of unipolar major depressive disorder. Arch Gen Psychiatry. 2000;57(4):375-80.

12. Cuijpers P, Dekker J, Hollon SD, Andersson G. Adding psychotherapy to pharmacotherapy in the treatment of depressive disorders in adults: a meta-analysis. J Clin Psychiatry. 2009;70(9):1219-29.

13. Beck AT. The current state of cognitive therapy: a 40-year retrospective. Arch Gen Psychiatry. 2005; 62(9):953-9.

14. Cuijpers P, Van Straten A, Bohlmeijer E, Hollon SD, Andersson G. The effects of psychotherapy for adult depression are overestimated: a meta-analysis of study quality and effect size. Psychol Med. 2010;40:21123.

15. Lequerica AH, Kortte K. Therapeutic engagement: a proposed model of engagement in medical rehabilitation. Am J Phys Med Rehabil. 2010;89(5):415-22.

16. Peeters F, Nicolson NA, Berkhof J, Delespaul P, deVries M. Effects of daily events on mood states in major depressive disorder. J Abnorm Psychol. 2003;112(2):203-11.

17. Peeters F, Berkhof J, Delespaul P, Rottenberg J, Nicolson NA. Diurnal mood variation in major depressive disorder. Emotion. 2006;6(3):383-91.

18. Denicoff KD, Ali SO, Sollinger AB, Smith-Jackson EE, Leverich GS, Post RM. Utility of the daily prospective National Institute of Mental Health Life-Chart Method (NIMH-LCM-p) ratings in clinical trials of bipolar disorder. Depress Anxiety. 2002;15(1):1-9.

19. Denicoff KD, Leverich GS, Nolen WA, Rush AJ, McElroy SL, Keck PE, et al. Validation of the prospective NIMH-Life-Chart Method (NIMH-LCM-p) for longitudinal assessment of bipolar illness. Psychol Med. 2000;30(6):1391-7.

20. Csikszentmihalyi M, Larson R. Validity and reliability of the Experience-Sampling Method. J Nerv Ment Dis. 1987;175(9):526-36.

21. Stone AA, Shiffman S. Ecological momentary assessment in behavioral medicine. Ann Behav Med. 1994;16:199-202.

22. Parati G, Omboni S, Bilo G. Why is out-of-office blood pressure measurement needed? Home blood pressure measurements will increasingly replace ambulatory blood pressure monitoring in the diagnosis and management of hypertension. Hypertension. 2009;54:181-7. 
23. Wexler R. Ambulatory blood pressure monitoring in primary care. South Med J. 2010;103(5):447-52.

24. Nardacci EA, Bode BW, Hirsch IB. Individualizing care for the many: the evolving role of professional continuous glucose monitoring systems in clinical practice. Diabetes Educ. 2010; 36(Suppl 1):4S-19S.

25. Bonato P. Clinical applications of wearable technology. Conf Proc IEEE Eng Med Biol Soc. 2009;2009:6580-3.

26. Bonato P. Advances in wearable technology and applications in physical medicine and rehabilitation. J Neuroeng Rehabil. 2005;2(1):2.

27. Groot PC. Patients can diagnose too: How continuous self-assessment aids diagnosis of, and recovery from, depression. J Ment Health. 2010;19(4):352-62.

28. Roy-Byrne P, Post RM, Uhde TW, Porcu T, Davis D. The longitudinal course of recurrent affective illness: life chart data from research patients at the NIMH. Acta Psychiatr Scand. 1985;317:1-34.

29. DeVries MW. The experience of psychopathology: investigating mental disorders in their natural settings. Cambridge: Cambridge University Press; 1992.

30. Trull TJ, Ebner-Priemer UW. Using experience sampling methods/ecological momentary assessment (ESM/EMA) in clinical assessment and clinical research: introduction to the special section. Psychol Assess. 2009;21(4):457-62.

31. Myin-Germeys I, Oorschot M, Collip D, Lataster J, Delespaul P, van Os J. Experience sampling research in psychopathology: opening the black box of daily life. Psychol Med. 2009;39(9):1533-47.

32. Delespaul P. Assessing schizophrenia in daily life: the experience sampling method. Maastricht, the Netherlands: Maastricht University Press; 1995.

33. Bolger N, Davis A, Rafaeli E. Diary methods: capturing life as it is lived. Annu Rev Psychol. 2003;54:579616.

34. Koster EH, De Raedt R, Leyman L, De Lissnyder E. Mood-congruent attention and memory bias in dysphoria: Exploring the coherence among information-processing biases. Behav Res Ther. 2010;48(3):219-25.

35. Ramel W, Goldin PR, Eyler LT, Brown GG, Gotlib IH, McQuaid JR. Amygdala reactivity and mood-congruent memory in individuals at risk for depressive relapse. Biol Psychiatry. 2007;61(2):231-9.

36. Fredrickson BL, Kahneman D. Duration neglect in retrospective evaluations of affective episodes. J Pers Soc Psychol. 1993;65(1):45-55.

37. Ben-Zeev D, Young MA. Accuracy of hospitalized depressed patients' and healthy controls' retrospective symptom reports: an experience sampling study. J Nerv Ment Dis. 2010;198(4):280-5.

38. Ebner-Priemer UW, Kuo J, Welch SS, Thielgen T, Witte S, Bohus M, et al. A valence-dependent groupspecific recall bias of retrospective self-reports: a study of borderline personality disorder in everyday life. J Nerv Ment Dis. 2006;194(10):774-9.

39. Herman S, Koran LM. In vivo measurement of obsessive-compulsive disorder symptoms using palmtop computers. Comput Hum Behav. 1998;14:449-62.

40. Ben-Zeev D, Young MA, Madsen JW. Retrospective recall of affect in clinically depressed individuals and controls. Cognition Emotion. 2009;23(5):1021-40.

41. Ram N, Gerstorf D. Time-structured and net intraindividual variability: tools for examining the development of dynamic characteristics and processes. Psychol Aging. 2009;24(4):778-91.

42. Molenaar CM, Campbell CG. The new person-specific paradigm in psychology. Curr Dir Psychol Sci. 2009;18(2):112-7.

43. Wichers M, Peeters F, Geschwind N, Jacobs N, Simons CJ, Derom C, et al. Unveiling patterns of affective responses in daily life may improve outcome prediction in depression: a momentary assessment study. J Affect Disord. 2010;124(1-2):191-5.

44. Anestis MD, Selby EA, Crosby RD, Wonderlich SA, Engel SG, Joiner TE. A comparison of retrospective selfreport versus ecological momentary assessment measures of affective lability in the examination of its relationship with bulimic symptomatology. Behav Res Ther. 2010;48(7):607-13.

45. Houtveen JH, LJ VAND. Negative affect and 24-hour ambulatory physiological recordings as predictors of spontaneous improvement of medically unexplained symptoms. Scand J Psychol. 2008;49(6):591-601.

46. Jacobs N, van Os J, Derom C, Thiery E, Delespaul P, Wichers M. Neuroticism explained? From a noninformative vulnerability marker to informative person-context interactions in the realm of daily life. $\mathrm{Br}$ J Clin Psychol. 2011;50(1):19-32. 
47. Wichers M, Aguilera M, Kenis G, Krabbendam L, Myin-Germeys I, Jacobs N, et al. The catechol-Omethyl transferase Val158Met polymorphism and experience of reward in the flow of daily life. Neuropsychopharmacology. 2008;33(13):3030-6.

48. Miller DJ, Vachon DD, Lynam DR. Neuroticism, negative affect, and negative affect instability: establishing convergent and discriminant validity using ecological momentary assessment. Pers Individ Dif. 2009;47(8):873-7.

49. Wenze SJ, Gunthert KC, Forand NR, Laurenceau JP. The influence of dysphoria on reactivity to naturalistic fluctuations in anger. J Pers. 2009;77(3):795-824.

50. Wichers M, Kenis G, Jacobs N, Myin-Germeys I, Schruers K, Mengelers R, et al. The psychology of psychiatric genetics: evidence that positive emotions in females moderate genetic sensitivity to social stress associated with the BDNF Val-sup-6-sup-6Met polymorphism. J Abnorm Psychol. 2008;117(3):699-704.

51. Jahng S, Wood PK, Trull TJ. Analysis of affective instability in ecological momentary assessment: Indices using successive difference and group comparison via multilevel modeling. Psychol Methods. 2008;13(4):354-75.

52. Geschwind N, Peeters F, Jacobs N, Delespaul P, Derom C, Thiery E, et al. Meeting risk with resilience: high daily life reward experience preserves mental health. Acta Psychiatr Scand. 2010;122(2):129-38.

53. Lardinois M, Lataster T, Mengelers R, Van Os J, Myin-Germeys I. Childhood trauma and increased stress sensitivity in psychosis. Acta Psychiatr Scand. 2011;123(1):28-35.

54. Henquet C, Rosa A, Delespaul P, Papiol S, Fananas L, van Os J, et al. COMT ValMet moderation of cannabisinduced psychosis: a momentary assessment study of 'switching on' hallucinations in the flow of daily life. Acta Psychiatr Scand. 2009;119(2):156-60.

55. Lataster T, Wichers M, Jacobs N, Mengelers R, Derom C, Thiery E, et al. Does reactivity to stress cosegregate with subclinical psychosis? A general population twin study. Acta Psychiatr Scand. 2009;119(1):45-53.

56. Wichers M, Lothmann C, Simons CJ, Nicolson NA, Peeters F. The dynamic interplay between negative and positive emotions in daily life predicts response to treatment in depression: a momentary assessment study. Br J Clin Psychol. 2012;51(2):206-22.

57. Wichers MC, Barge-Schaapveld DQ Nicolson NA, Peeters F, de Vries M, Mengelers R, et al. Reduced stresssensitivity or increased reward experience: the psychological mechanism of response to antidepressant medication. Neuropsychopharmacology. 2009;34(4):923-31.

58. Geschwind N, Nicolson NA, Peeters F, van Os J, Barge-Schaapveld D, Wichers M. Early improvement in positive rather than negative emotion predicts remission from depression after pharmacotherapy. Eur Neuropsychopharmacol. 2011;21(3):241-247.

59. Peeters F, Berkhof J, Rottenberg J, Nicolson NA. Ambulatory emotional reactivity to negative daily life events predicts remission from major depressive disorder. Behav Res Ther. 2010;48(8):754-60.

60. Barge-Schaapveld DQ Nicolson NA. Effects of antidepressant treatment on the quality of daily life: an experience sampling study. J Clin Psychiatry. 2002;63(6):477-85.

61. Wichers MC, Myin-Germeys I, Jacobs N, Peeters F, Kenis G, Derom C, et al. Evidence that moment-tomoment variation in positive emotions buffer genetic risk for depression: a momentary assessment twin study. Acta Psychiatr Scand. 2007;115(6):451-7.

62. Wichers M, Myin-Germeys I, Jacobs N, Peeters F, Kenis G, Derom C, et al. Genetic risk of depression and stress-induced negative affect in daily life. Br J Psychiatry. 2007;191:218-23.

63. Ebner-Priemer UW, Trull TJ. Ambulatory assessment: an innovative and promising approach for clinical psychology. Eur Psychol. 2009;14(2):109-19.

64. Depp CA, Mausbach B, Granholm E, Cardenas V, Ben-Zeev D, Patterson TL, et al. Mobile interventions for severe mental illness: design and preliminary data from three approaches. J Nerv Ment Dis. 2010;198(10):715-21.

65. Heron KE, Smyth JM. Ecological momentary interventions: incorporating mobile technology into psychosocial and health behavior treatments. Br J Health Psychol. 2010;15(1):1-39.

66. Newman MG, Consoli AJ, Taylor CB. A palmtop computer program for the treatment of generalized anxiety disorder. Behav Modif. 1999;23(4):597-619.

67. Przeworski A, Newman MG. Palmtop computer-assisted group therapy for social phobia. J Clin Psychol. 2004;60(2):179-88. 
68. Clark LA, Watson D. Tripartite model of anxiety and depression: psychometric evidence and taxonomic implications. J Abnorm Psychol. 1991;100(3):316-36.

69. Wichers M, Jacobs N, Derom C, Thiery E, Van Os J. Depression: too much negative affect or too little positive affect? Twin Res Hum Genet. 2007;10 (Suppl):19-20.

70. Wichers M, Hartmann JA, Kramer IMA, Lothmann C, Peeters F, van Bemmel L, et al. Translating assessments of the film of daily life into person-tailored feedback interventions in depression. Acta Psychiatr Scand. 2011;123(5):402-3.

71. van Beljouw I, Verhaak P, Prins M, Cuijpers P, Penninx B, Bensing J. Reasons and determinants for not receiving treatment for common mental disorders. Psychiatr Serv. 2010;61(3):250-7.

72. Paykel ES. Partial remission, residual symptoms, and relapse in depression. Dialogues Clin Neurosci. 2008;10(4):431-7.

73. Stassen HH, Angst J, Hell D, Scharfetter C, Szegedi A. Is there a common resilience mechanism underlying antidepressant drug response? Evidence from 2848 patients. J Clin Psychiatry. 2007;68(8):1195-205. 


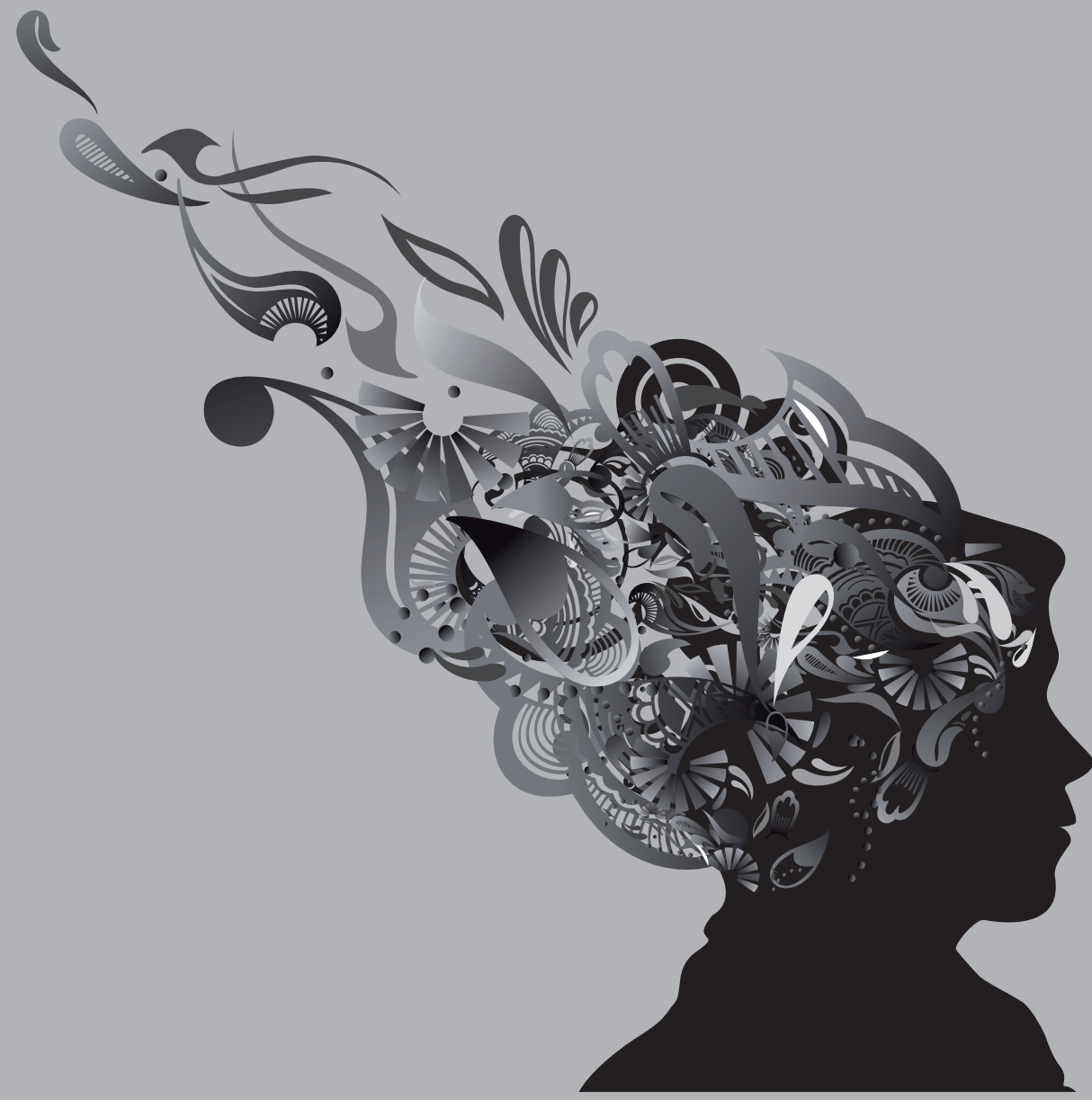




\title{
CHAPTER 5
}

\section{A therapeutic application of the experience}

\section{sampling method in the treatment of depression:}

\author{
a randomized controlled trial
}

I. Kramer ${ }^{1,2^{*}}$, CJP. Simons ${ }^{1,2^{*}}$, JA. Hartmann ${ }^{1,2}$, C. Menne-Lothmann ${ }^{2}$, W. Viechtbauer ${ }^{2}$, F. Peeters ${ }^{2}$, K. Schruers ${ }^{2}$, AL. van Bemmel ${ }^{1,2}$, I. Myin-Germeys², P. Delespaul', , J. van Os ${ }^{2,4}$, M. Wichers ${ }^{2}$

${ }^{1}$ GGzE, Institute of Mental Health Care Eindhoven and the Kempen, Eindhoven, the Netherlands ${ }^{2}$ Department of Psychiatry and Psychology, South Limburg Mental Health Research and Teaching Network, EURON, Maastricht University, the Netherlands ${ }^{3}$ Mondriaan Mental Health Trust, South Limburg, the Netherlands

${ }^{4}$ King's College London, Department of Psychosis Studies, Institute of Psychiatry, London, UK

* Contributed equally 


\section{Abstract}

Background: In depression, the ability to experience daily life positive affect predicts recovery and reduces relapse rates. Interventions based on the experience sampling method (ESM-I) are ideally suited to provide insight in personal, contextualized patterns of positive affect. The aim of this study was to examine whether add-on ESM-derived feedback on personalized patterns of positive affect is feasible and useful to patients, and results in a reduction of depressive symptomatology.

Methods: Depressed outpatients $(\mathrm{n}=102)$ receiving pharmacological treatment participated in a randomized controlled trial with three arms: an experimental group receiving add-on ESM-derived feedback, a pseudo-experimental group participating in ESM but receiving no feedback, and a control group. The experimental group participated in an ESM procedure (three days per week over a 6-week period) using a palmtop. This group received weekly standardized feedback on personalized patterns of positive affect. Hamilton Depression Rating Scale - 17 (HDRS) and Inventory of Depressive Symptoms (IDS) scores were obtained before and after the intervention. During a 6-month follow-up period, five HDRS and IDS assessments were completed.

Results: Add-on ESM-derived feedback resulted in a significant and clinically relevant stronger decrease in HDRS score relative to the control group $(p<0.01 ;-5.5$ point reduction in HDRS at 6 months). Compared to the pseudo-experimental group, a clinically relevant decrease in HDRS score was apparent at 6 months $(B=-3.6, p=0.053)$. Self-reported depressive complaints (IDS) yielded the same pattern over time. The use of ESM-I was deemed acceptable and the provided feedback easy to understand. Patients attempted to apply suggestions from ESM-derived feedback to daily life.

Conclusions: These data suggest that the efficacy of traditional passive pharmacological approach to treatment of major depression can be enhanced by using person-tailored daily life information regarding positive affect. 


\section{Introduction}

According to the World Health Organization, depression is among the leading causes of disability (1). Improving the efficacy of pharmacotherapy and psychotherapy is considered a priority. Enlarging the window of observation of depressive symptomatology to out-of-the-office situations could result in a more detailed and personalized assessment of contextual influences on symptomatology, and hence may add to the effect of existing treatments.

Self-monitoring, comprising once-a-day retrospective paper-pencil assessments of mood, has been shown to reduce depressive symptomatology (2-4). However, because retrospectively obtained self-assessments are biased by mood-congruent emotional and cognitive biases (5), the use of prospective in-the-moment daily life assessments may be used to improve reliability, providing a much more fine-grained film of the dynamics of depressive symptomatology, which may aid in optimizing treatment decisions. Furthermore, digital instead of paper-and-pencil assessments have the advantage that data are immediately available.

Digitalized prospective, in-the-moment monitoring is commonly used in medical disciplines. Continuous monitoring, for example, is used in the treatment of hypertension and diabetes (i.e., $24 \mathrm{~h}$ blood pressure or plasma glucose monitoring). In the field of mental health, however, this area remains to be explored. For mental health outcomes, the equivalent of mobile ambulatory assessment of medical outcomes has recently become available in the form of electronic momentary assessment techniques. These techniques represent the combination of experience sampling methodology (ESM) with new electronic tools, such as the PsyMate (6), allowing for direct electronic recording of the data. ESM consists of repeated assessments of affective experience and context in the flow of daily life (7-9).

Until recently, ESM has been used only in the context of research to identify momentto-moment patterns and mechanisms of psychopathology (10-14). With the advent of personal digital assistants (PDAs) and web-based applications, however, real-life data are immediately available to patients and professional caregivers. This creates the possibility for ESM interventions (ESM-I) that can transform implicit real-life dynamic patterns to explicit, visualized and quantifiable configurations, through which dysfunctional patterns become modifiable. ESM-I has the additional benefit that it can be easily implemented in standard mental health care and does not require much additional investment of clinicians $(6,11)$. Therefore, ESM-I constitutes a new viable approach to improve personalized mental health care and stands to become a widely used mobile-health tool in clinical practice $(10-13,15)$. 
A new and exciting development is the use of real-life self-monitoring with ESM-I in depressed patients to gain insight in personalized patterns of positive affect and the context in which they are experienced. Numerous recent studies (16-19) have shown the importance of the reward system and positive affect experience in resilience against depression. It was demonstrated that especially positive affect - more than its counterpart, negative affect - is crucial and necessary in predicting recovery from depression (20-23). Furthermore, a recent randomized controlled trial showed that allocation to an intervention that increased real-life positive affect experience was associated with a significant decline in depressive symptoms (22). Therefore, the next step in the treatment of depression is to examine whether self-monitoring can be used as an intervention to increase insights in personalized patterns of positive affect. Personalized feedback focused on positive affect and its context may help both the patient and the professional carer in their search for custom opportunities to increase the experience of that affect, thus enabling recovery from depression.

Although the above arguments suggest that ESM-I represents a novel approach with a potential to improve treatment in mental health care, feasibility and patient preference need to be considered as well. There is a need to know how patients experience this procedure and whether they are able and willing to participate.

Therefore, the aims of the current study were to examine whether: a) ESM-derived personalized feedback can be used, in combination with standard antidepressant medication, as an effective add-on treatment for depressive symptoms designed to increase patients' resources with regard to positive affective experience; b) ESM-I is considered feasible and useful by patients.

To our knowledge, this is the first randomized controlled trial using ESM as a novel therapeutic intervention in depressed patients, with a view to improve personalized treatment.

\section{Methods}

\section{Participants and design}

Consecutively presenting depressed outpatients attending mental health care facilities serving the catchment areas of the Dutch cities of Eindhoven and Maastricht were approached by their health care professionals and recruited into the study. In addition, recruitment in the same catchment areas was also carried out independent of contact with mental health care services by distributing posters and flyers in health care facilities and local media. Recruitment occurred between January 2010 and February 2012.

Inclusion criteria were: age 18-65 years; a DSM-IV-TR diagnosis of depressive episode with a current total score on the Hamilton Depression Rating Scale - 17 (HDRS)(24) of at 
least 8 (i.e., above remission cut-off and including residual depressive states); receiving pharmacological treatment with antidepressants or mood stabilizers; adequate vision; sufficient Dutch language skills; no current or lifetime diagnosis of non-affective psychotic disorder, and no (hypo)manic or mixed episode within the past month.

The study protocol was approved by the Medical Ethics Committee of Maastricht University Medical Centre. Informed consent was obtained from all participants. The trial was registered at Netherlands Trial Register (Identifier: NTR1974).

A randomized controlled trial was conducted with three treatment arms. After baseline, patients were randomly allocated to the experimental, pseudo-experimental or control group. In addition to treatment as usual (TAU), the experimental group participated in an ESM procedure (three days per week over a 6-week period) using a palmtop. This group received weekly standardized feedback on personalized patterns of positive affect. Feedback was given to both the patient and the mental health professional. The pseudo-experimental group also participated in the ESM procedure (three days per week over a 6-week period) in addition to TAU, but without feedback. The control group received no additional intervention during TAU.

Randomization (allocation ratio 1:1:1) was stratified according to duration of antidepressant pharmacotherapy (new/switch vs. maintenance, i.e. receiving antidepressant or mood stabilizing medication for less vs. longer than 8 weeks prior to study entry), and current psychotherapy (yes or no). The randomization sequence in blocks of six (using the sequence generator on the Internet site random.org) was generated by the first author of this paper. An independent research assistant wrote the randomization code into sealed numbered envelopes. After completion of all baseline assessments, the interviewer allocated participants to their treatment condition based on the randomization code in the sealed envelope (opened in order of sequence). Interviewers were not blind to the patients' treatment allocation.

\section{Procedure}

Figure 1 shows participant flow and procedure throughout the trial. The study protocol consisted of a telephone interview, a screening, a baseline assessment (week 0), a sixweek intervention period (weeks 1 to 6), a post assessment (week 7), and five follow-up assessments (at weeks 8, 12, 16, 20, 32). From baseline (week 0) onward, the overall study duration was 32 weeks.

The recruitment process started with a short telephone interview conducted by a psychologist or psychiatrist to establish whether inclusion criteria were likely met. During the screening, the Structured Clinical Interview for DSM-IV Axis I Disorders (SCID-I) (25), the HDRS, and the 30-item Inventory of Depressive Symptoms (IDS-SR)(26) were administered. The HDRS semi-structured interview and the IDS self-report questionnaire, 
both assessing the severity of depressive symptoms, were completed at baseline, at post assessment and at follow-up. The IDS was used as a measure that is independent from the interpretation of the interviewer. ESM assessments took place as part of the baseline assessment (week 0), during the 6-week intervention period (weeks 1 to 6), and at the post assessment (week 7). The feasibility of the ESM measurement procedure and the desirability of the ESM-derived feedback on positive affect were evaluated through specific questions, with items rated on 7-point Likert scales (1="not at all" to 7="very").

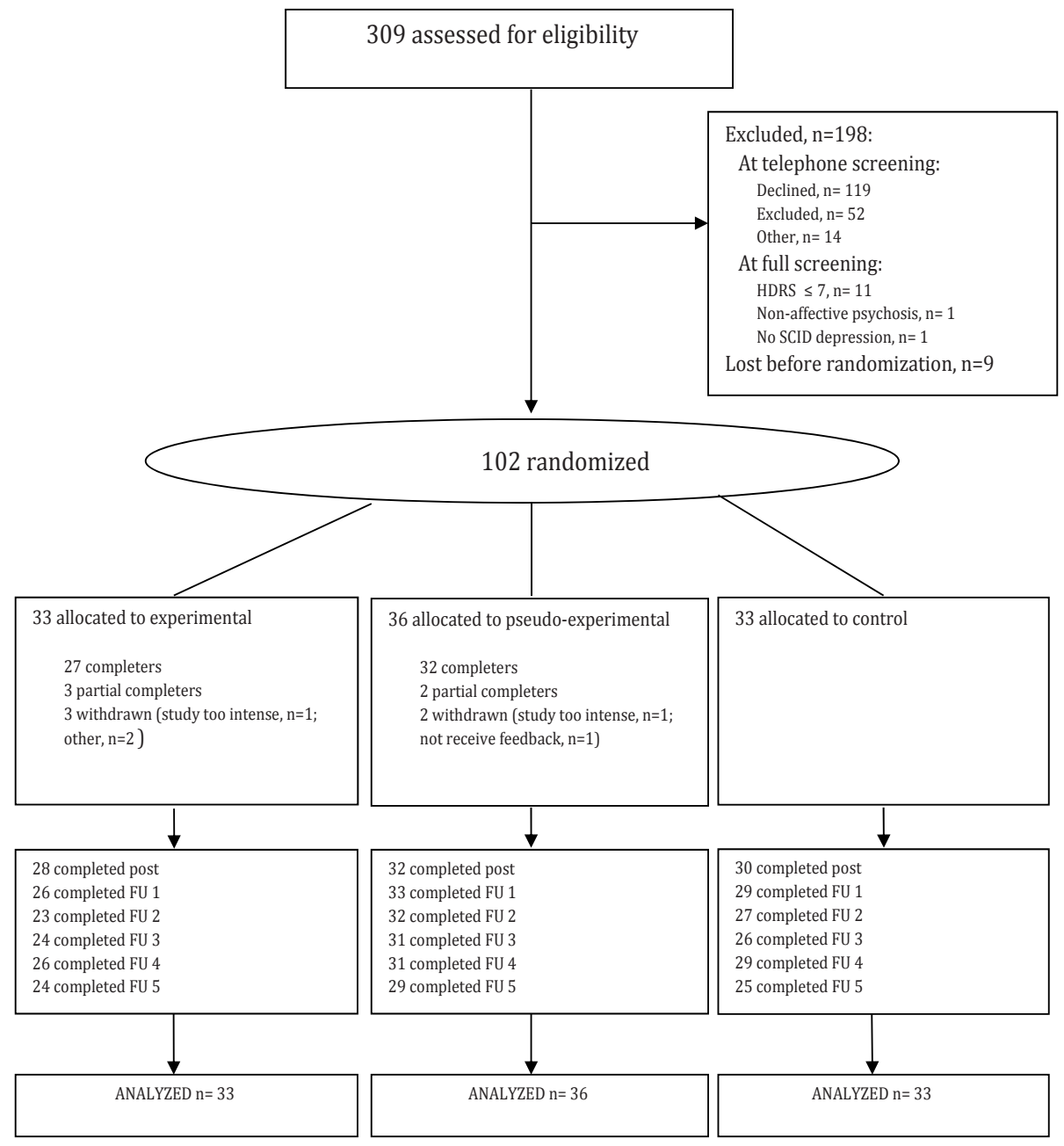

Figure 1 | Flowchart of the study. HDRS - Hamilton Depression Rating Scale - 17, SCID Structured Clinical Interview for DSM-IV Axis I Disorders, post - immediate post assessment, FU - follow-up assessment 
ESM was carried out according to previous studies (7, 27-29). The recently developed PsyMate, a palmtop, was used to digitally collect daily life momentary assessments of positive affect in relation to momentary context and activity. The PsyMate was programmed to emit a beep 10 times per day at random intervals in each of ten 90min time blocks between 7.30 and 22.30. At each beep, participants used the PsyMate to digitally complete a brief beep-questionnaire including current affect (four positive affect and six negative affect items) as well as current context and activities ("daily life activities", "persons present", "physical activity", and "events"). PsyMate positive affect indicators included the adjectives "cheerful", "satisfied", "enthusiastic" and "relaxed" (22). Negative affect was indexed by the adjectives "down", "suspicious", "guilty", "irritated", "lonely" and "anxious". The self-assessments were rated on 7-point Likert scales (ranging from 1="not at all" to 7="very"). Participants were instructed to complete the beep-questionnaire as quickly as possible after the beep.

During both the 5-day ESM baseline assessment and the 5-day ESM post assessment, 10 beep-questionnaires were generated per day. The total number of beepquestionnaires therefore was 50 for both the ESM baseline and ESM post assessments. During the 6-week intervention period, participants completed 10 beep-questionnaires per day for three consecutive days $(10 \times 3 \times 6=180$ beep-questionnaires).

The ESM procedure was explained in an initial briefing session, and a practice run was performed to ensure that the participants understood the questions and the device. The debriefing to assess aspects of feasibility of the ESM procedure with PsyMate was scheduled immediately after the ESM baseline assessment.

\section{Intervention}

The experimental group received standardized ESM-derived feedback. Feedback sessions immediately followed the weekly ESM procedure. In these face-to-face sessions, feedback was provided by the researcher (a psychologist or psychiatrist). The feedback on participants' momentary affective state in specific daily life contexts and the association with depressive symptoms was given verbally, in writing and graphically (Figure 2). Feedback showed actual levels of positive affect (the mean of the items "cheerful", "satisfied", "enthusiastic" and "relaxed") in the context of daily life activities (Figure 2a and 2b), events and social situations. The second part of the feedback showed changes in positive affect level (Figure 2c) and the number of depressive complaints over the course of the ESM intervention.

The ESM-derived feedback was divided in three modules. In each module, a novel element of feedback was added cumulatively. The first feedback sessions (1 and 2) were focused on positive affect experienced during activities. Feedback sessions 3 and 4 additionally focused on positive affect experienced after daily events, differentiating 
A

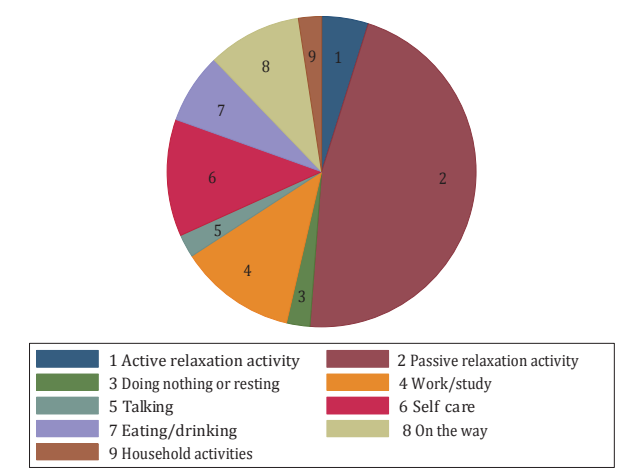

B

Active relaxation activity

Passive relaxation activity

Doing nothing or resting

Work/study

Talking

Self care

Eating/drinking

On the way

Household activities

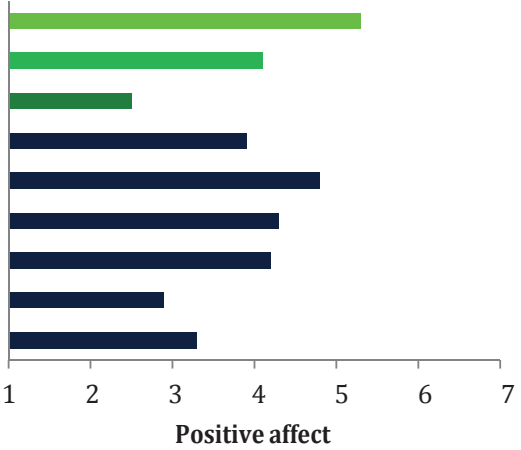

C

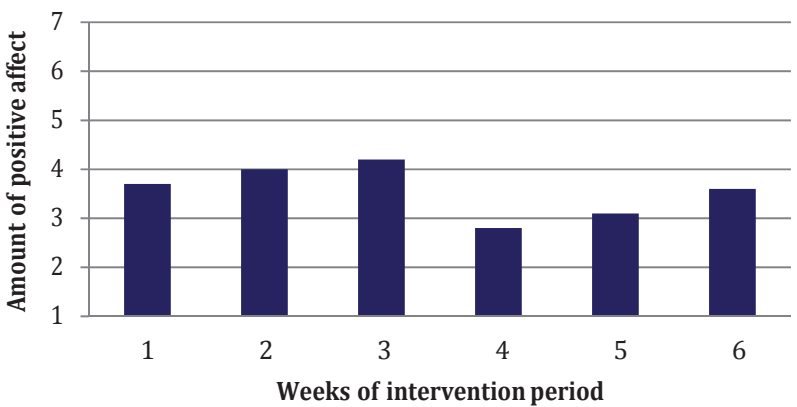

Figure 2 | Examples of feedback graphs. (A) Amount of time spent doing different types of activities. (B) Amount of positive affect experienced per type of activity. (C) Mean level of positive affect over the 6-weeks intervention period. 
between affect experienced during events appraised with an internal vs. external locus of control. Finally, feedback sessions 5 and 6 additionally focused on positive affect experienced during social interactions in daily life.

Participants' opinion about the feedback procedures was evaluated at the post assessment.

The pseudo-experimental group was similar in procedure to the experimental group except that no feedback was given. To prevent any effects of different duration of the sessions, this group's sessions were filled with an alternative activity (an additional HDRS interview).

\section{Statistical analysis}

Statistical analyses were conducted using STATA 12.1 (30). The data had a hierarchical structure, because multiple assessments of HDRS and IDS depressive symptoms were clustered within patients.

First, to examine the impact of treatment allocation on course of depressive symptoms, mean HDRS total scores were plotted over time (in weeks from baseline to last follow-up) for each of the three groups. The best fit was provided by a linear model (time) for the experimental and control group and a polynomial model (time and time ${ }^{2}$ ) for the pseudo-experimental group.

Next, the XTMIXED command was used to perform a multilevel regression analysis with the two-way interaction between time (in weeks) and treatment allocation as fixed effects, patients as random intercept and a random slope for time. The covariance was set to unstructured. The LINCOM command was used to calculate estimated betweengroup effects. A difference of three or more points on the HDRS was a priori considered as clinically relevant $(31,32)$.

Power calculations using the STATA (30) SAMPSI command were based on previous work (33), and led to an initial sample size of 120 with a power of $84 \%$ to detect a 3 -point difference in HDRS score $(31,32)$. However, because many participants were excluded, inclusion rate was lower than expected. The eventual number of patients who participated in the trial was 102.

\section{Results}

The characteristics of the included subject sample at screening are shown in Table 1. There were no large or significant differences in socio-demographic characteristics between the groups, but at screening there were some differences in clinical features. Compared to the pseudo-experimental and control group, patients in the experimental group used lithium more often and displayed lower HDRS and IDS total scores (Table 
1). Group differences in HDRS and IDS or lithium use were non-significant at baseline (two weeks later, i.e. just before start of the intervention) $(F(2 ; 98)=1.00, p=0.37$; $\mathrm{F}(2 ; 98)=1.52, \mathrm{p}=0.22$, and $\chi^{2}(2)=4.65, \mathrm{p}=0.10$, respectively).

Of the 102 randomized patients, 93 completed at least one HDRS assessment during the post-intervention assessment period of approximately 6 months. There were no large or significant between-group differences in completion of at least one HDRS assessment during this post-intervention assessment period $\left(\chi^{2}(2)=0.93, p=0.62\right)$. Similar findings were obtained for the IDS $\left(\chi^{2}(2)=0.93, p=0.62\right)$.

Of the 69 patients allocated to the experimental or pseudo-experimental group, 59 (85.5\%) completed the 6-week intervention period, comprising 6x3 ESM assessment days and six corresponding intervention sessions. There was no large or significant difference in baseline depressive symptoms between patients who fully completed the intervention period and those who did not (HDRS: $B=0.76, p=0.72$; IDS: $B=1.03, p=0.80$ ). The average number of completed beep-questionnaires in these 59 patients was $135.5 \pm 16.5$ out of 180 , indicating a completion rate of $75.3 \%$. There were no significant differences between the experimental vs. the pseudo-experimental group in either the mean number of completed beep-questionnaires over the entire intervention period $(t=0.91, d f=57, p=0.18)$, or the number of patients who completed all six intervention sessions $\left(\chi^{2}(1)=0.69, p=0.50\right)$. Feedback sessions lasted significantly longer (mean: $48.9 \pm 11.2 \mathrm{~min}$, range $27-105 \mathrm{~min}$ ) compared to the pseudo-experimental interview sessions (mean: 39.5 $\pm 12.9 \mathrm{~min}$, range $15-90 \mathrm{~min})(B=9.57, \mathrm{p}<0.001)$.

Figure 3 displays the results of the multilevel regression analysis of the interaction between treatment allocation and time on HDRS and IDS scores. The experimental group demonstrated a significantly greater weekly decline in depressive symptoms over the complete study period compared to the control group (HDRS: $\mathrm{B}=-0.15, \mathrm{p}<0.001$; IDS: $\mathrm{B}=-$ $0.29, \mathrm{p}=0.002$ ). Between-group comparisons demonstrated that the decline in depressive symptoms in the experimental group compared to the control group became significant at week 8 (IDS) and 11 (HDRS) and lasted until the end of the study (week 32). Over time, differences between the experimental and control group became stronger, reaching a -5.5 HDRS point difference and a -13.1 IDS point difference at week 32.

The pseudo-experimental group followed a different pattern: it displayed significantly lower HDRS and IDS scores compared to the control group, starting directly after the intervention period (week 7). However, the initial decrease in depressive symptoms did not persist until the last assessment: after week 26 (HDRS) and week 28 (IDS), the difference in depressive symptoms between the pseudo-experimental group and the control group was no longer significant (Figure 3). Compared to the pseudo-experimental group, patients in the experimental group demonstrated clinically relevant lower HDRS scores, a priori defined as a decrease of 3 or more points $(31,32)$, at the end of the study 


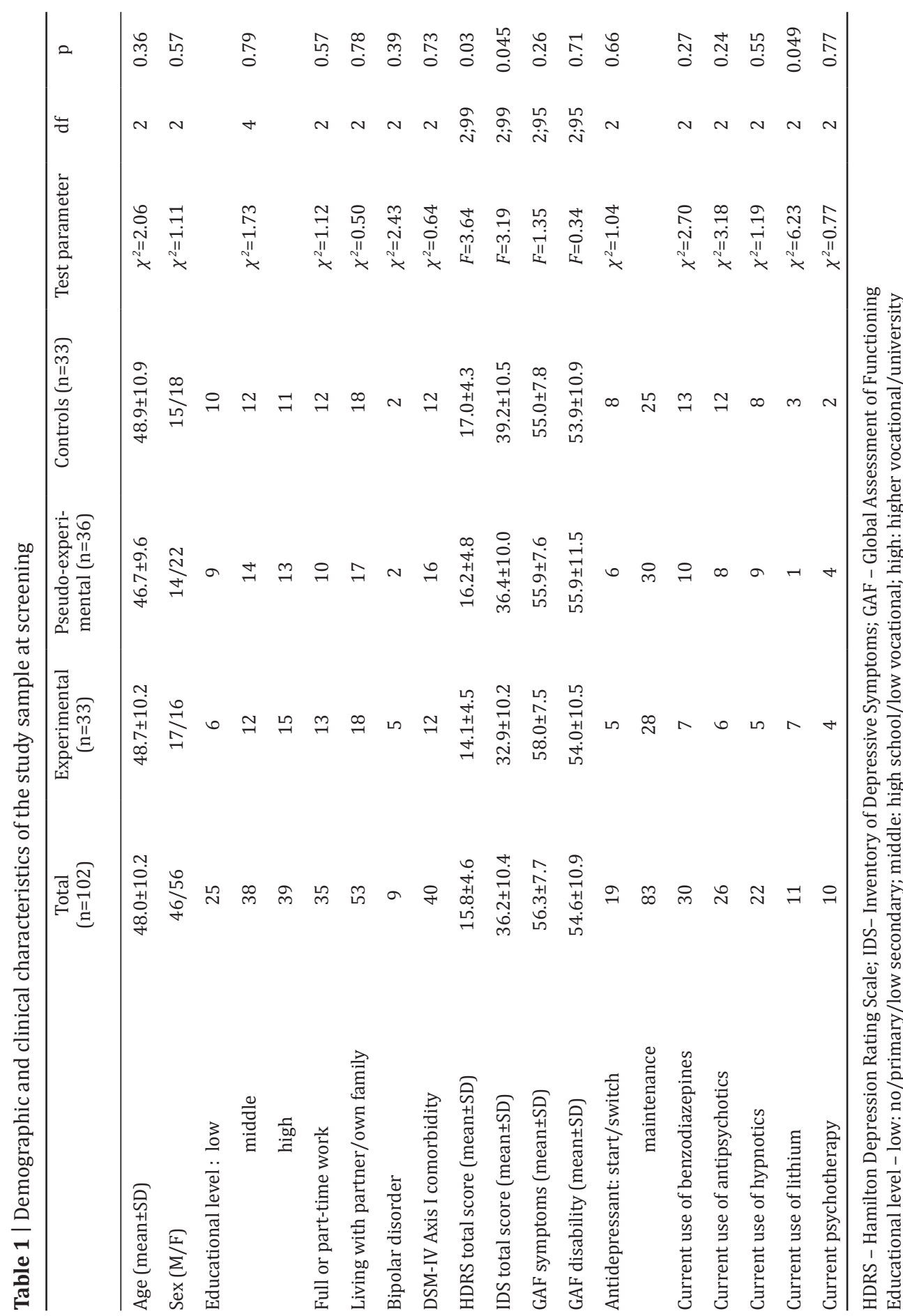


A

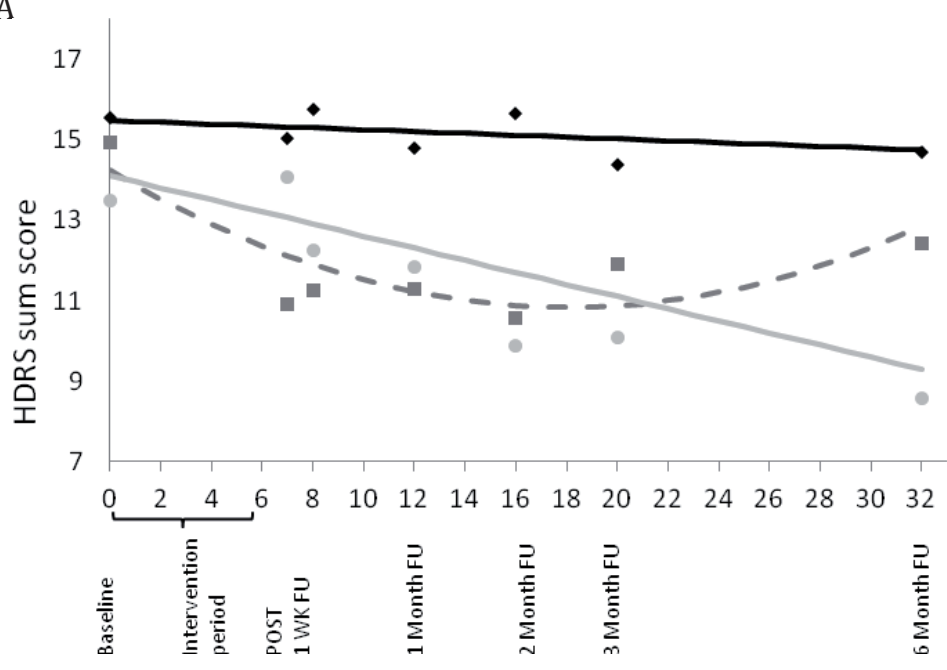

- control

- pseudoexperimental

- experimental

— predicted control

- - predicted pseudoexperimental predicted experimental

Time since baseline (in weeks)

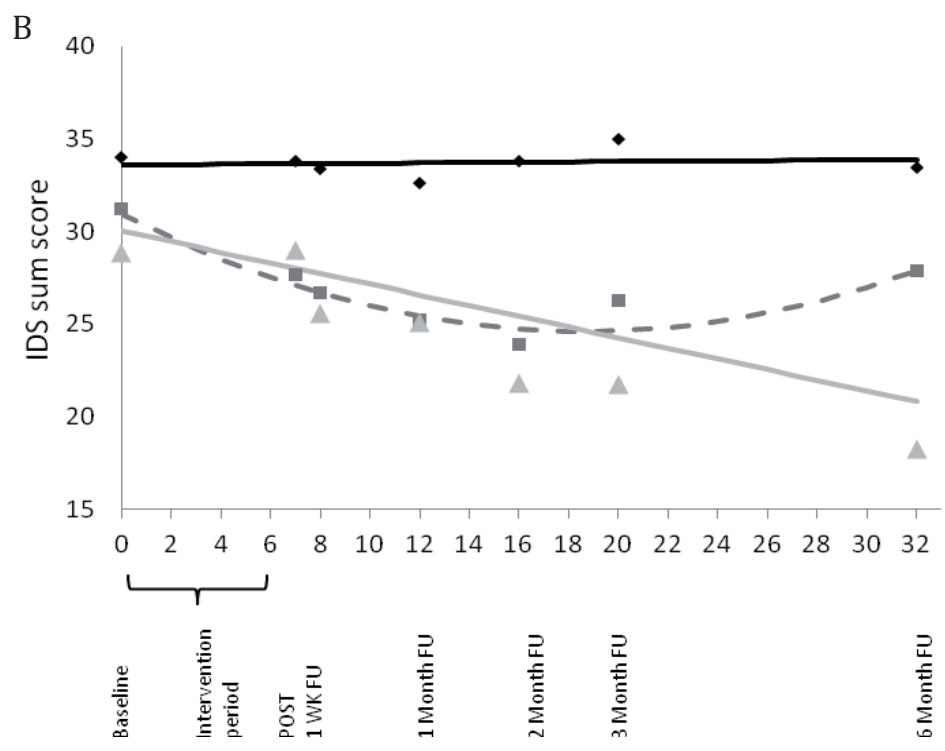

- control

- pseudoexperimental

A experimental

prediced control

- - predicted pseudoexperimental predicted experimental

Time since baseline (in weeks)

Figure 3 | Mean depression scores and predicted lines plotted over time. (A) Hamilton Depression Rating Scale (HDRS). (B) Inventory of Depressive Symptoms (IDS). POST - immediate post assessment, FU - follow-up assessment 
(weeks 31 and 32 ) ( $\mathrm{B}=-3.1, \mathrm{p}=0.08$ and $\mathrm{B}=-3.6, \mathrm{p}=0.053$, respectively).

Patients in the control group did not demonstrate a change in HDRS and IDS scores over the course of the study $(B=-0.02, p=0.56$, and $B=0.01, p=0.92$, respectively).

Table 2 displays the results of the patient estimated feasibility of ESM-I. Results indicated that the procedure was not very stressful with respect to number of beeps per day, time to fill out a beep-questionnaire, or sound of the beep. Instructions on how to use the PsyMate were rated as very clear.

Table 2 | Patient estimated feasibility of the procedure and opinions on the feedback

Mean (min-max) $\quad$ SD $\mathrm{N}$ (subjects)
(scale 1-7)

\section{Feasibility of the procedure}

Was the text on screen readable?

Was it difficult to switch PsyMate on?

Was the PsyMate difficult to control?

Were the verbal instructions you received about using the PsyMate clear?

Were the written instructions you received with the PsyMate clear?

Were the questions you answered on the PsyMate difficult or unclear?

Did you find it annoying or stressful to use the PsyMate?

With respect to the number of beeps per day?

With respect to the time it took to answer the questions for a single beep?

With respect to the noises/sound volume?

$\begin{array}{lll}5.8(2-7) & 1.4 & 102 \\ 1.6(1-6) & 1.2 & 102 \\ 1.4(1-5) & 0.8 & 102 \\ 6.6(3-7) & 0.7 & 102 \\ 6.5(1-7) & 1.0 & 96 \\ 2.6(1-6) & 1.5 & 102 \\ & & \\ 3.1(1-7) & 1.6 & 102 \\ 2.5(1-7) & 1.5 & 102 \\ 2.0(1-7) & 1.5 & 102\end{array}$

\section{ESM-derived feedback}

I found it easy to understand the explanation given with the feedback

The researcher was able to answer my questions well when there was something I didn't understand

I was annoyed that I wasn't allowed to get answers about (help) questions that were about my specific problems

Would you like to have received more specific advice following the feedback you were given?

I appreciated getting a summary of the feedback

I found it easy to understand the feedback summary

I was happy to get feedback in the form of graphs

I found it easy to understand the information in the graphs

I have tried to apply the suggestions from the feedback in my daily life

The amount of information in the feedback was exactly right

The duration of the contact reserved for feedback was exactly right

$\begin{array}{lll}6.1(4-7) & 0.6 & 25 \\ 6.4(6-7) & 0.5 & 24 \\ 2.3(1-6) & 1.6 & 22 \\ 3.2(1-6) & 2.0 & 25 \\ 6.2(5-7) & 0.7 & 25 \\ 6.2(3-7) & 0.9 & 25 \\ 6.5(4-7) & 0.7 & 24 \\ 6.3(3-7) & 0.9 & 25 \\ 5.4(3-7) & 1.1 & 25 \\ 5.4(4-7) & 0.9 & 25 \\ 6.2(4-7) & 0.4 & 25\end{array}$


Table 2 also displays the results regarding participants' opinions on ESM-derived feedback, indicating that feedback on positive affect was relatively easy to understand. Also, participants appreciated getting ESM-derived feedback and tried to apply the suggestions from the feedback to their daily lives.

\section{Discussion}

This study shows that the use of add-on momentary assessment technology may be effective as a therapeutic tool to complement standard antidepressant treatment. Allocation to the intervention group with ESM-derived feedback on positive affect was associated with a linear decrease in HDRS depressive symptoms over time that persisted until the last follow-up six months later. The difference with the pseudo-experimental group was clinically relevant and borderline significant.

Although the use of ESM-derived feedback in the treatment of depression has been suggested before $(8,10,11,34,35)$, the present endeavor is, to our knowledge, the first randomized controlled trial that systematically examined ESM-I as a therapeutic tool to provide depressed patients with insight into personalized patterns of positive affect. Relative to receiving passive pharmacological treatment only, depressed patients who received additional feedback on personalized opportunities for positive affect demonstrated a clinically relevant and persistent decrease in depressive symptomatology. This could reflect increased insight and accompanying change towards behavioral patterns increasing positive affect. In contrast, the effects in the pseudoexperimental group (self-monitoring without feedback) did not appear to persist. Because these patients may have thought that they were receiving the experimental intervention, this could reflect a placebo response. Another speculation is that these findings result from a short-lived behavioral activation effect attributable to the weekly in-the-office appointments in the intervention period. Moreover, these appointments, in which patients had the opportunity to share their depressive feelings, may have been experienced as more supportive than the experimental feedback appointments, which would explain the stronger immediate reduction in symptoms in this group. Finally, although speculative, the effect of continuous self-monitoring (i.e. without ESMderived feedback) on depressive symptoms may also be explained by an increased momentary emotional awareness (36). This may make ESM-I an interesting tool to use in mindfulness based cognitive therapy, as suggested by Telford et al (36).

Although interventions based on momentary assessment technology have been developed for several mental disorders and health promoting behaviors $(15,37$ 43), actual implementation in mental health care is still limited (12). Examples are interventions directed to practice anxiety reducing techniques (39), remind patients 
to use previously learned skills (43), or remind patients about medication adherence (41). Interventions that provided insights derived from momentary assessments were developed for attention deficit-hyperactivity disorder (38) and migraine attacks (44).

Mild to severely depressed patients $(45,46)$ were able and motivated to complete ESM measurements for a longer period of time (18 days), and became actively involved in their recovery process by trying to implement suggestions derived from ESM-feedback into their personal daily life. So, the current results suggest that ESM interventions as an add-on treatment may be both feasible and effective for patients suffering from mild to severe depressive disorder, including residual depressive states that are associated with substantial morbidity (47-52).

A first limitation of this study is that neither patients nor researchers were blind with regard to treatment allocation. If knowledge of allocation resulted in biased depression ratings by the patient one would, in contrast to the current results, expect that the experimental group (relative to the control group) demonstrated the largest decrease in HDRS depression at the post-intervention assessment. Knowledge of allocation by researchers did not result in biased HDRS depression ratings, because analyses using the IDS self-rating depression scale yielded similar results. Second, although we showed clinically significant effect size differences between the experimental and the pseudoexperimental group, these differences were not conclusive by conventional alpha. This may relate to the fact that the sample was somewhat smaller $(n=102)$ compared to the sample size $(n=120)$ required to obtain power of $>0.80$. Finally, given that more faceto-face time may reduce depressive complaints, the longer duration (approximately 10 min) of the feedback sessions may have had an influence on the results. However, given the resemblance between the two groups with respect to weekly ESM assessments and subsequent weekly face-to-face contact with the researcher, it is unlikely that this had a large impact on the results.

Using ESM-I in mental health care has the potential to bridge the gap between the therapist office and patients' daily life, by bringing the patients' daily life into the therapist office, and creating the opportunity to extend the therapeutic setting to patients' daily life. The latter may be achieved by a web-based interactive ESM-I application that provides in-the-moment feedback based on previously assessed individual patterns of affect and behavior. This may result in helpful person-tailored insights that not only foster individualized therapy but also the diagnostic process, monitoring of early change in response to medication alterations, or identifying individual affective patterns indicating recovery or relapse (53). This approach could be integrated with cognitive behavior therapy (54) and may create a 24/7 access to and provision of care. Currently, web-based interactive ESM-I applications are in development and studies are required to examine treatment efficacy as well as cost-efficiency. 
Although the present findings suggest that providing ESM-derived feedback to depressed patients is feasible and leads to a lasting decrease in depressive symptomatology, these results need to be replicated.

\section{Acknowledgements}

M. Wichers was supported by the Netherlands Organization for Scientific Research (VENI grant no. 916.76.147). The study was funded by the Dutch Health Research Council (ZON-MW (grants nos. 171001002 and 91501003). The tool with which momentary assessments were performed (the PsyMate) is developed under the auspices of the Maastricht University technology transfer office, partially supported by unrestricted grants from Servier and Janssen-Cilag. The research leading to these results has received funding from the European Community's Seventh Framework Program under grant agreement no. HEALTH-F2-2009-241909 (Project EU-GEI). The authors thank all patients for participating and all collaborating mental health centers for their support in recruiting patients. They also thank W. Beuken and T. Driessen for administrative support, and P. Höhn, K. Akkermans, R. Diederen, K. Gielen, M. Hendriks and E. Pols for support in data acquisition. The first two authors contributed equally to this work. 


\section{References}

1. World Health Organization. Depression. A global public health concern. Geneva, Switzerland: World Health Organization. 2012.

2. Dimidjian S, Hollon SD, Dobson KS, Schmaling KB, Kohlenberg RJ, Addis ME, et al. Randomized trial of behavioral activation, cognitive therapy, and antidepressant medication in the acute treatment of adults with major depression. J Consult Clin Psychol. 2006;74(4):658-70.

3. Fuchs CZ, Rehm LP. A self-control behavior therapy program for depression. J Consult Clin Psychol. 1977;45(2):206-15.

4. Lewinsohn PM. A behavioral approach to depression. In: RJ Friedman \& Katz M, editor. The psychology of depression: contemporary theory and research. Oxford, England: Wiley; 1974. p 157-78.

5. Koster EHW, De Raedt R, Leyman L, De Lissnyder E. Mood-congruent attention and memory bias in dysphoria: exploring the coherence among information-processing biases. Behav Res Ther. 2010;48(3):219-25.

6. Myin-Germeys I, Birchwood M, Kwapil T. From environment to therapy in psychosis: a real-world momentary assessment approach. Schizophr Bull. 2011;37(2):244-7.

7. Csikszentmihalyi M, Larson R. Validity and reliability of the Experience-Sampling Method. J Nerv Ment Dis. 1987;175(9):526-36.

8. aan het Rot M, Hogenelst K, Schoevers RA. Mood disorders in everyday life: a systematic review of experience sampling and ecological momentary assessment studies. Clin Psychol Rev. 2012;32(6):51023.

9. Myin-Germeys I, Oorschot M, Collip D, Lataster J, Delespaul P, van Os J. Experience sampling research in psychopathology: opening the black box of daily life. Psychol Med. 2009;39:1533-47.

10. Wichers M, Hartmann JA, Kramer IMA, Lothmann C, Peeters F, van Bemmel L, et al. Translating assessments of the film of daily life into person-tailored feedback interventions in depression. Acta Psychiatr Scand. 2011;123(5):402-3.

11. Wichers M, Simons CJP, Kramer IMA, Hartmann JA, Lothmann C, Myin-Germeys I, et al. Momentary assessment technology as a tool to help patients with depression help themselves. Acta Psychiatr Scand. 2011;124(4):262-72.

12. Trull TJ, Ebner-Priemer U. Ambulatory assessment. Annu Rev Clin Psychol. 2013;9(1):151-76.

13. Trull TJ, Ebner-Priemer UW. Using experience sampling methods/ecological momentary assessment (ESM/EMA) in clinical assessment and clinical research: introduction to the special section. Psychol Assessment. 2009;21(4):457-62.

14. Thewissen V, Bentall RP, Oorschot $\mathrm{M}$, à Campo J, van Lierop T, van Os J, et al. Emotions, self-esteem, and paranoid episodes: an experience sampling study. Br J Clin Psychol. 2011;50(2):178-95.

15. Heron KE, Smyth JM. Ecological momentary interventions: incorporating mobile technology into psychosocial and health behaviour treatments. Br J Health Psychol. 2010;15(1):1-39.

16. Wichers MC, Barge-Schaapveld DQCM, Nicolson NA, Peeters F, de Vries M, Mengelers R, et al. Reduced stress-sensitivity or increased reward experience: the psychological mechanism of response to antidepressant medication. Neuropsychopharmacology. 2009;34(4):923-31.

17. Wichers M, Peeters F, Geschwind N, Jacobs N, Simons CJP, Derom C, et al. Unveiling patterns of affective responses in daily life may improve outcome prediction in depression: a momentary assessment study. J Affect Disord. 2010;124(1-2):191-5.

18. Dunn BD. Helping depressed clients reconnect to positive emotion experience: current insights and future directions. Clin Psychol Psychother. 2012;19(4):326-40.

19. Garland EL, Fredrickson B, Kring AM, Johnson DP, Meyer PS, Penn DL. Upward spirals of positive emotions counter downward spirals of negativity: insights from the broaden-and-build theory and affective neuroscience on the treatment of emotion dysfunctions and deficits in psychopathology. Clin Psychol Rev. 2010;30(7):849-64.

20. Geschwind N, Nicolson NA, Peeters F, van Os J, Barge-Schaapveld D, Wichers M. Early improvement in positive rather than negative emotion predicts remission from depression after pharmacotherapy. Eur Neuropsychopharmacol. 2011;21(3):241-7. 
21. Dichter GS, Felder JN, Petty C, Bizzell J, Ernst M, Smoski MJ. The effects of psychotherapy on neural responses to rewards in major depression. Biol Psychiatry. 2009;66(9):886-97.

22. Geschwind N, Peeters F, Drukker M, van Os J, Wichers M. Mindfulness training increases momentary positive emotions and reward experience in adults vulnerable to depression: a randomized controlled trial. J Consult Clin Psychol. 2011;79(5):618-28.

23. Fredrickson BL, Cohn MA, Coffey KA, Pek J, Finkel SM. Open hearts build lives: positive emotions, induced through loving-kindness meditation, build consequential personal resources. J Pers Soc Psychol. 2008;95(5):1045.

24. Hamilton M. A rating scale for depression. J Neurol Neurosurg Psychiatry. 1960;23:56-62.

25. First M, Spitzer R, Gibbon M, Williams J. SCID-I. Structured clinical interview for DSM-IV Axis I disorders [SCID I. Gestructureerd klinisch interview voor DSM-IV As-I stoornissen]. Lisse, the Netherlands: Swets \& Zeitlinger; 1998.

26. Rush AJ, Gullion CM, Basco MR, Jarrett RB, Trivedi MH. The Inventory of Depressive Symptomatology (IDS): psychometric properties. Psychol Med. 1996;26(3):477-86.

27. Delespaul P. Assessing schizophrenia in daily life: the Experience Sampling Method. Maastricht, the Netherlands: Maastricht University Press; 1995.

28. Wichers M, Myin Germeys I, Jacobs N, Peeters F, Kenis G, Derom C, et al. Genetic risk of depression and stress-induced negative affect in daily life. Br J Psychiatry. 2007:218-23.

29. Myin Germeys I, van Os J, Schwartz JE, Stone AA, Delespaul PA. Emotional reactivity to daily life stress in psychosis. Arch Gen Psychiatry. 2001;58(12):1137-44.

30. StataCorp. Stata Statistical Software: Release 12. College Station, TX, USA: Statacorp LP; 2011.

31. Hegerl U, Mergl R. The clinical significance of antidepressant treatment effects cannot be derived from placebo-verum response differences. J Psychopharmacol. 2010;24(4):445-8.

32. NICE. Depression: management of depression in primary and secondary care. Clinical Practice Guideline No. 23. London, UK: National Institute for Clinical Excellence; 2004.

33. Barge Schaapveld DQ Nicolson NA. Effects of antidepressant treatment on the quality of daily life: an experience sampling study. J Clin Psychiatry. 2002;63(6):477-85.

34. Palmier-Claus J. The clinical uses of momentary assessment. Acta Psychiatr Scand. 2011;124(4):241-2.

35. Ebner-Priemer UW, Trull TJ. Ecological momentary assessment of mood disorders and mood dysregulation. Psychol Assessment. 2009;21(4):463-75.

36. Telford C, McCarthy-Jones S, Corcoran R, Rowse G. Experience sampling methodology studies of depression: the state of the art. Psychol Med. 2012;42(6):1119-29.

37. Hareva DH, Okada H, Kitawaki T, Oka H. Supportive intervention using a mobile phone in behavior modification. Acta Med Okayama. 2009;63(2):113-20.

38. Tryon WW, Tryon GS, Kazlausky T, Gruen W, Swanson JM. Reducing hyperactivity with a feedback actigraph: initial findings. Clin Child Psychol Psychiatry. 2006;11(4):607-17.

39. Newman MG, Kenardy J, Herman S, Barr Taylor C. Comparison of palmtop-computer-assisted brief cognitive-behavioral treatment to cognitive-behavioral treatment for panic disorder. J Consult Clin Psychol. 1997;65(1):178-83.

40. Litt MD, Kadden RM, Kabela-Cormier E. Individualized assessment and treatment program for alcohol dependence: results of an initial study to train coping skills. Addiction. 2009;104(11):1837-8.

41. Granholm E, Ben-Zeev D, Link PC, Bradshaw KR, Holden JL. Mobile Assessment and Treatment for Schizophrenia (MATS): a pilot trial of an interactive text-messaging intervention for medication adherence, socialization, and auditory hallucinations. Schizophr Bull. 2012;38(3):414-25.

42. Miklowitz DJ, Price J, Holmes EA, Rendell J, Bell S, Budge K, et al. Facilitated Integrated Mood Management for adults with bipolar disorder. Bipolar Disord. 2012;14(2):185-97.

43. Depp CA, Mausbach B, Granholm E, Cardenas V, Ben-Zeev D, Patterson TL, et al. Mobile interventions for severe mental illness: design and preliminary data from three approaches. J Nerv Ment Dis. 2010;198(10):715-21.

44. Sorbi MJ, Mak SB, Houtveen JH, Kleiboer AM, van Doornen LJP. Mobile web-based monitoring and coaching: feasibility in chronic migraine. J Med Internet Res. 2007;9(5). 
45. Zimmerman M, Martinez JH, Friedman M, Boerescu DA, Attiullah N, Toba C. How can we use depression severity to guide treatment selection when measures of depression categorize patients differently? J Clin Psychiatry. 2012;73(10):1287-91.

46. American Psychiatric Association Task Force for the Handbook of Psychiatric Measures. Handbook of Psychiatric Measures. Washington, DC: American Psychiatric Association; 2000.

47. Cuijpers P, de Graaf R, van Dorsselaer S. Minor depression: risk profiles, functional disability, health care use and risk of developing major depression. J Affect Disord. 2004;79(1):71-9.

48. Judd LL, Akiskal HS, Maser JD, Zeller PJ, Endicott J, Coryell W, et al. Major depressive disorder: a prospective study of residual subthreshold depressive symptoms as predictor of rapid relapse. J Affect Disord. 1998;50(2):97-108.

49. Rush A, Trivedi M, Wisniewski S, Nierenberg A, Stewart J, Warden D, et al. Acute and longer-term outcomes in depressed outpatients requiring one or several treatment steps: a STAR* D report. Am J Psychiatry. 2006;163(11):1905-17.

50. Cuijpers P, Vogelzangs N, Twisk J, Kleiboer A, Li J, Penninx BW. Differential mortality rates in major and subthreshold depression: meta-analysis of studies that measured both. Br J Psychiatry. 2013;202(1):22-7.

51. Cuijpers P, Smit F, Van Straten A. Psychological treatments of subthreshold depression: a meta-analytic review. Acta Psychiatr Scand. 2007;115(6):434-41.

52. Ormel J, Petukhova M, Chatterji S, Aguilar-Gaxiola S, Alonso J, Angermeyer MC, et al. Disability and treatment of specific mental and physical disorders across the world. Br J Psychiatry. 2008;192(5):368-75.

53. van Os J, Delespaul P, Wigman J, Myin-Germeys I, Wichers M. Beyond DSM and ICD: introducing "precision diagnosis" for psychiatry using momentary assessment technology. World Psychiatry. 2013;12:113-7.

54. Kelly J, Gooding P, Pratt D, Ainsworth J, Welford M, Tarrier N. Intelligent real-time therapy: harnessing the power of machine learning to optimise the delivery of momentary cognitive-behavioural interventions. J Ment Health. 2012;21(4):404-14. 


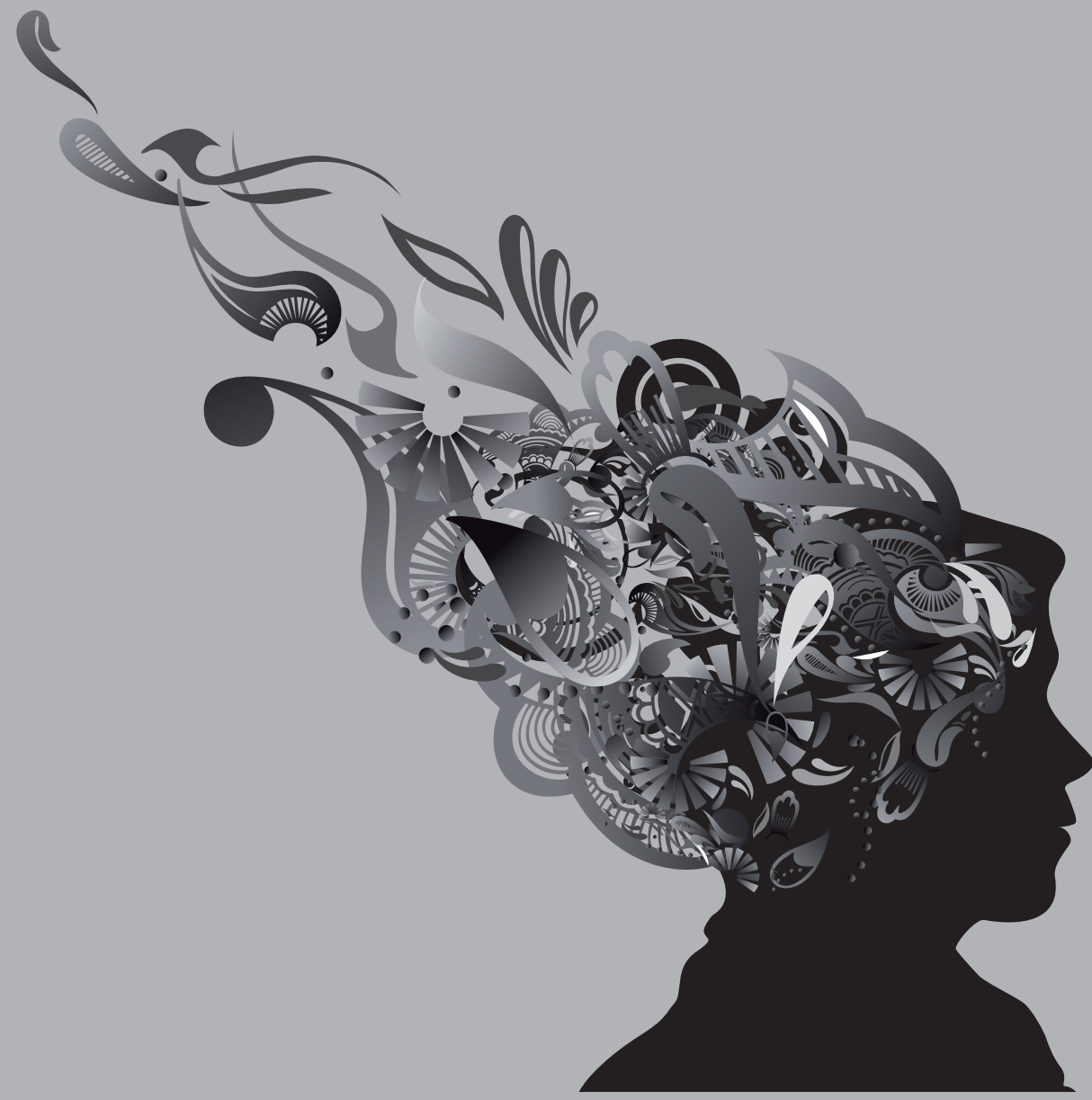




\section{CHAPTER 6}

General Discussion 
The aim of this thesis was to (i) present research examining the relevance of emotional experiences at the micro-level as a source for a better understanding of mechanisms underlying the development of symptoms of a mental disorder, and (ii) explore whether a shift from macro-level symptomatology to micro-level emotional experiences may have a place in the treatment of mental disorders.

In the first part, we started with an examination of the affective pathway to psychosis on a macro-level of psychotic symptomatology, after which a shift to the micro-level of daily life emotional experiences was made (chapter 2-3). It was demonstrated that a genetic liability for depression moderated the impact of childhood adversity on macro-level future psychotic symptomatology. Furthermore, macro-level depressive symptomatology mediated this association (chapter 2). The impact of depressive symptomatology on psychosis could be traced back to the micro-level of daily life emotional experiences. At the micro-level of daily life dynamics, we demonstrated that an increase in daily life negative affect (NA) resulted in a 3-hour lasting increase in paranoia. Childhood trauma and stress sensitivity were identified as risk factors that were related to the increase in paranoia during the NA increase. Depressive symptoms, on the other hand, were related to the persistence of paranoid experiences. Finally it was demonstrated that paranoid experiences following the NA increase predicted future development of psychotic symptoms (chapter 3).

In the second part of this thesis, we explored whether assessing daily life emotional experiences could contribute to the daily practice of mental health care by personalizing the diagnostic process and treatment of major depression (chapter 4). A new intervention to increase daily life positive affect (PA) in depression was developed and its potential to increase the effectiveness of antidepressant treatment was examined (chapter 5). An ESM-based intervention (ESM-I) was developed to provide depressive patients with personalized feedback on their individual patterns of PA. It was shown that patients receiving personalized feedback on patterns of PA demonstrated, relative to the control group, a significant and clinically relevant reduction in depressive symptoms over the 6-month follow-up period.

The present thesis thus shifted from the macro-level of symptomatology to the microlevel of the 'smallest building blocks' of emotional experiences and explored whether the examination of daily life emotional experiences could lead to new insights in developmental pathways that could also contribute to the development of effective interventions.

In the following, the findings of the present thesis are discussed. First, the results of the first part (chapter 2 and 3) are discussed and placed in the context of recent models of psychopathology. Second, the results of the clinical application part (chapter 
4 and 5) are discussed. Valorization, clinical implications and future directions will be discussed in the next chapter.

\section{ESM as a source for a better understanding of mechanisms underlying psychopathology}

A wealth of literature exists on the effects of risk factors and mechanisms in the development of macro-level psychopathology. While macro-level examinations can reveal whether certain risk factors are important in the development of psychopathology, questions regarding (i) how and why these risk factors impact on emotional experiences and behavioral patterns, and (ii) temporal emotional dynamics remain unanswered. Refining the level of examination from macro-level symptomatology to micro-level emotional experiences and behavioral patterns provides a fruitful approach to study etiological questions in a higher level of detail.

For almost three decades, ESM has been used in the context of research to identify moment-to-moment patterns and mechanisms underlying psychopathology. This has resulted in a wealth of knowledge on etiology and phenomenology of psychopathology $(1,2)$, e.g. affective (3-8) and psychotic disorders (9-11). Following this line of work, in the present thesis, ESM was used as a technique to explore affective mechanisms underlying the development of psychosis (chapter 2 and 3). Figure 1 summarizes and integrates the findings from chapter $\mathbf{2}$ and 3 (see also chapter 3 ). It shows schematically how and why childhood adversity, stress sensitivity, and affective symptoms may exert their influence on later macro-level course of psychotic symptoms by their impact on micro-level hour-to-hour dynamics between NA and paranoia. The shift to the microlevel of daily life emotional dynamics thus not only revealed temporal NA-paranoia dynamics but also suggested how risk factors changed the micro-level of hour-to-hour interplay between NA and paranoia by increasing the amplitude or persistence of paranoid experiences following NA boosts. These subtle changes in repetitive everyday emotional dynamics were found to be associated with follow-up SCL-90-R and CAPE psychotic symptoms, suggesting that if these psychotic symptoms, where to become persistent, they may constitute a risk factor for later clinical symptoms, help-seeking behavior and finally the development of a clinical psychotic disorder (12-14). The persistence of paranoid experiences following the NA boost was associated with the presence of current depressive symptomatology. This finding is in line with earlier work that showed that psychotic experiences and symptoms more often occur in a context of negative mood states (15-17). 


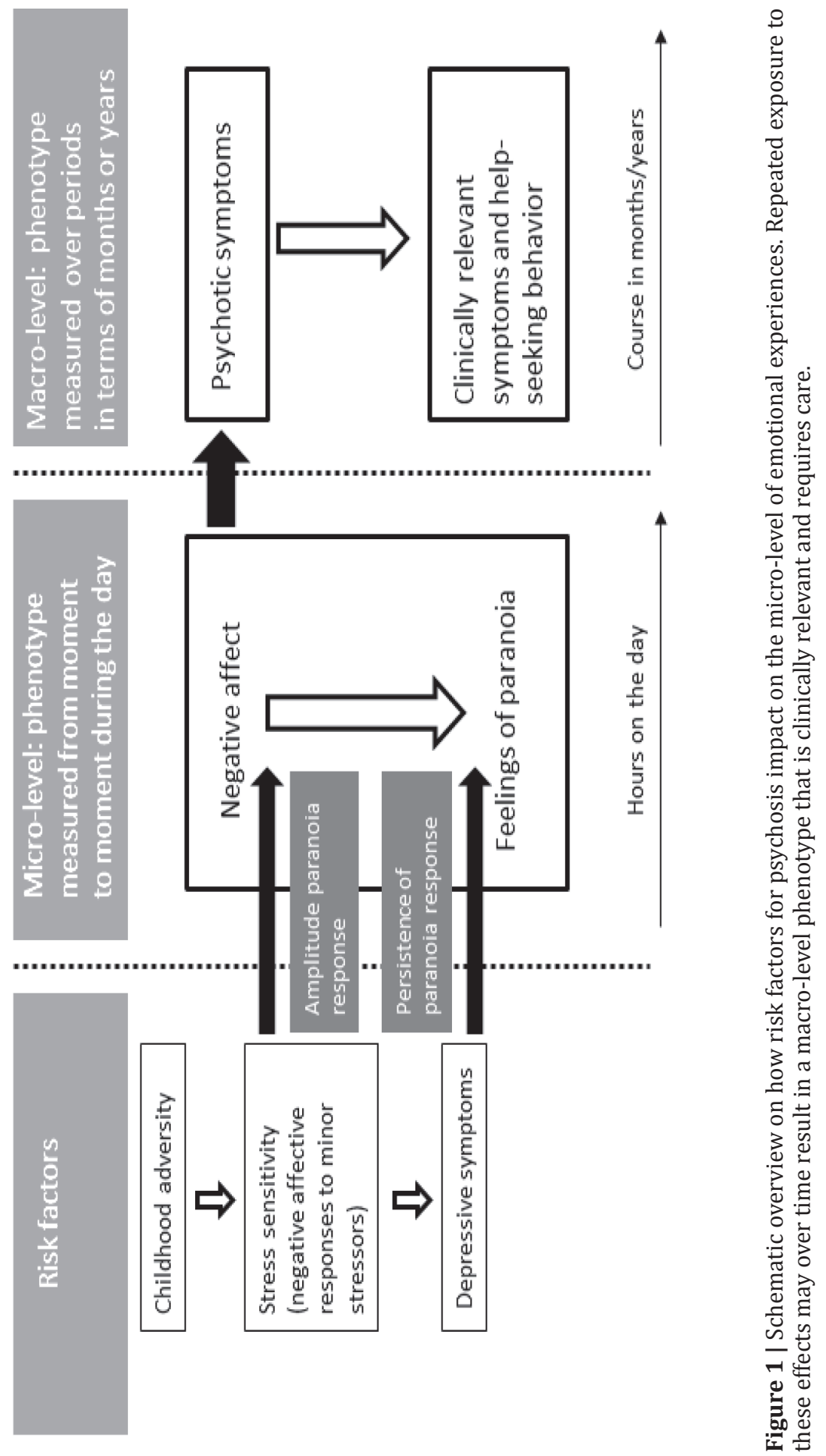


The question can thus be raised whether depressive symptomatology in the context of psychotic symptomatology represents an independent co-morbid latent condition sharing environmental and genetic risk factors with psychosis or that depressive and psychotic experiences and symptomatology are potentially simultaneously occurring experiences that co-influence and possibly trigger each other. The current work provides support for the latter hypothesis, as it was not only demonstrated that affective experiences and symptomatology preceded the development of psychotic experiences and macro-level psychotic symptomatology (chapter 2 and 3), but also that micro-level dynamics were present between NA and paranoia. A boost in NA led to a 3-hours lasting increase in paranoia (chapter 3). Using ESM, it was thus shown that (i) psychotic experiences tend to occur more often in a context of negative mood states and (ii) emotional experiences may trigger each other over time.

Figure 1 reflects a continuum which ranges from non-clinical a-specific emotional experiences and (subclinical) symptoms to full blown psychotic symptoms that may result in help-seeking behavior. Over time, an individual can make the transition from the non-clinical to the clinical phenotype. Analogue to somatic medicine, e.g. oncology, a clinical staging model of psychopathology has recently been presented (18-20). This model ranges from early non-specific mental distress resulting in (aberrant) emotional experiences and behavioral patterns to clinical psychiatric syndromes. Depending on the patterns of these dynamic and mutually impacting experiences over time, an individual may make to transition to the clinical stage. Using the staging perspective, stage appropriate treatment options can be provided.

In contrast to the incorporated phenotypical variation in staging models, current diagnostic systems assume that symptoms of a mental disorder reflecta stable underlying entity or disorder. This assumption leaves little room for individual differences in symptomatology or variation over time. Symptoms (and disorders) are considered to be rather dichotomous; they are present or not. This all or nothing assumption has little resemblance to the observed heterogeneity of symptomatology and disorders in daily practice in which individuals experience (sub)clinical symptoms that vary over time, react to the environment, co-influence each other and possibly giving rise to new symptoms $(18,21-25)$.

Network theory, on the other hand, proposes that a cluster of symptoms arise, not as a representation of a single underlying liability or construct that triggers a set of independent symptoms, but as a reflection of the notion that symptoms can trigger and co-influence each other, forming a circuitry of causal relationships $(20,21,26$ 28). Symptoms are thus interconnected in a network in which connections between different symptoms vary in intensity. Moreover, the strength of the interconnections 
can vary over time, as symptoms can get worse and trigger new symptoms as well, and between individuals reflecting the heterogeneity in clinical presentation of for example depression or psychosis. Networks can reveal the dynamic patterns between the smallest building blocks of symptomatology, the daily life emotional experiences and behavioral patterns (26). Therefore, using ESM to zoom in the micro-level of experience may help to elucidate mechanisms underlying psychopathology which may provide a fruitful approach in the search of the nature and etiology of psychopathology.

\section{From etiology to intervention: using ESM in the treatment of mental disorders}

Until recently, ESM has mainly been used for research purposes. Current technological progress, however, has resulted in the possibility to collect electronic or digital ESM assessments by means of a digital ESM device (PDA, app's for mobile phones or tablets). In contrast to the traditional paper-pencil approach with booklets and digital wristwatches, with the advent of digital ESM devices, the results of continuous assessments of symptoms of a mental disorder become available for both patient and clinician. This creates the possibility to assess emotional experiences, behavioral patterns and symptomatology on a reliable, ecological valid individual way that takes variation over time and variation in response to the environment into account (29). Hence, using ESM in the daily practice could complement traditional in-the-office (retrospective) assessments of macro-level symptomatology with a fine-grained and detailed account of individual daily life information of emotional experiences and behavioral patterns. Consequently, ESM could help in deconstructing the black box of psychopathology into small manageable pieces of emotion and behavior (chapter 4). This personal information, collected on the micro-level of experience may reveal to the individual not only why he/she has developed certain symptoms but also temporal relationships between certain emotional experiences, behaviors and symptoms. ESM thus renders the opportunity to 'personalize' the diagnostic process (chapter $\mathbf{4},(21,26,29,30)$ ) by providing an individualized and highly detailed picture of the dynamics of a disorder in a single person. This individual-specific knowledge may also pinpoint to what symptoms and behaviors an intervention should be targeted to in order to increase its therapeutic relevance. Consequently, this creates the possibility for 'personalized treatment' $(26,29$, 30). A potentially interesting starting point for intervention in daily life dynamics is positive affect (PA) (26). Daily life PA is known to predict recovery from depression, and it protects against a relapse of a depressive episode $(7,31-33)$. According to a recent network account of depression, PA potentially plays an important part in its dynamic nature (26). Hence, directing a therapeutic intervention towards PA could potentially counteract negative dynamics between affect and behavioral tendencies. For example, by increasing one's PA, the tendency for social engagement could be enhanced possibly 
leading to a decrease in negative affect (26). In such a network, PA could possibly reflect a 'positive node' (defined as a node that reflects a protective factor in the development of psychopathology) (27). A positive node may be an essential link in the switch from mental ill health to mental health.

Therefore, an intervention based on ESM (ESM-I) was developed to increase daily life PA in depressed patients receiving anti-depressant medication to potentially induce a series of changes eventually hypothesized to lead to emotional and behavioral change resulting in a reduction in depressive symptomatology (chapter 5). Due to the boost in momentary PA, the attentional focus might have become enlarged which possibly resulted in a behavioral and motivational change. If repeatedly experienced, this change might have led to an ongoing increase in positive affect leading to the demonstrated clinical relevant and persistent reduction in depressive symptomatology. Yet, the exact mechanism by which the depressed patients demonstrated the reduction in depressive symptomatology still needs to be examined in detail in future work.

An interesting and unexpected finding was the observed temporary reduction in depressive symptomatology in the group that received continuous monitoring (thus without feedback) combined with weekly in-the-office sessions. Although this temporarily reduction in depressive symptomatology may be the result from behavioral activation due to the weekly in-the-office visits or reflects a placebo response, it is also plausible that continuous monitoring results in an increased focus on the actual moment of completing the ESM questionnaire and hence increase one's self-awareness and self-observation. As such it may be used in mindfulness trainings or Mindfulness Based Cognitive Therapy, as has been suggested before (34).

Furthermore, long-term monitoring (an 8-week period) was considered feasible by mild to severe depressed patients (chapter 5). These results support its clinical relevance and application possibilities. Examples are continuous monitoring of emotional experiences, behavior and symptomatology to optimize treatment effect, and monitoring of medication discontinuation strategies.

In sum, the potential contribution of ESM to clinical practice thus ranges from refining the diagnostic process (ESM-D), ESM based interventions (ESM-I), to continuous monitoring of for example treatment effects (ESM-M)(chapter 4, 5 (21, 26, 29, 30, 35)). 


\section{References}

1. Myin-Germeys I, Oorschot M, Collip D, Lataster J, Delespaul P, van Os J. Experience sampling research in psychopathology: opening the black box of daily life. Psychol Med. 2009;39(9):1533-47.

2. Heron KE, Smyth JM. Ecological momentary interventions: incorporating mobile technology into psychosocial and health behaviour treatments. Br J Health Psychol. 2010;15(1):1-39.

3. Wichers M, Schrijvers D, Geschwind N, Jacobs N, Myin-Germeys I, Thiery E, et al. Mechanisms of geneenvironment interactions in depression: evidence that genes potentiate multiple sources of adversity. Psychol Med. 2009;39(7):1077-86.

4. Wichers M, Myin Germeys I, Jacobs N, Peeters F, Kenis G, Derom C, et al. Genetic risk of depression and stress-induced negative affect in daily life. Br J Psychiatry. 2007;191(3):218-23.

5. Wichers M, Geschwind N, Jacobs N, Kenis G, Peeters F, Derom C, et al. Transition from stress sensitivity to a depressive state: longitudinal twin study. Br J Psychiatry. 2009;195(6):498-503.

6. Wichers M, Peeters F, Geschwind N, Jacobs N, Simons CJP, Derom C, et al. Unveiling patterns of affective responses in daily life may improve outcome prediction in depression: a momentary assessment study. J Affect Disord. 2010;124(1-2):191-5.

7. Geschwind N, Nicolson NA, Peeters F, van Os J, Barge-Schaapveld D, Wichers M. Early improvement in positive rather than negative emotion predicts remission from depression after pharmacotherapy. Eur Neuropsychopharmacol. 2011;21(3):241-7.

8. Wichers MC, Barge-Schaapveld DQCM, Nicolson NA, Peeters F, de Vries M, Mengelers R, et al. Reduced stress-sensitivity or increased reward experience: the psychological mechanism of response to antidepressant medication. Neuropsychopharmacology. 2009;34(4):923-31.

9. Myin Germeys I, van Os J, Schwartz JE, Stone AA, Delespaul PA. Emotional reactivity to daily life stress in psychosis. Arch Gen Psychiatry. 2001;58(12):1137-44.

10. Myin Germeys I, Delespaul P, van Os J. Behavioural sensitization to daily life stress in psychosis. Psychol Med. 2005;35(5):733-41.

11. Oorschot M, Lataster T, Thewissen V, Wichers M, Myin-Germeys I. Mobile assessment in schizophrenia: a data-driven momentary approach. Schizophr Bull. 2012;38(3):405-13.

12. Wigman JTW, van Winkel R, Raaijmakers QAW, Ormel J, Verhulst FC, Reijneveld SA, et al. Evidence for a persistent, environment-dependent and deteriorating subtype of subclinical psychotic experiences: a 6-year longitudinal general population study. Psychol Med. 2011;41(11):2317-29.

13. van Os J, Linscott RJ. Introduction: the extended psychosis phenotype-relationship with schizophrenia and with ultrahigh risk status for psychosis. Schizophr Bull. 2012;38(2):227-30.

14. van Os J, Linscott RJ, Myin-Germeys I, Delespaul P, Krabbendam L. A systematic review and meta-analysis of the psychosis continuum: evidence for a psychosis proneness-persistence-impairment model of psychotic disorder. Psychol Med. 2009;39(2):179-95.

15. Buckley PF, Miller BJ, Lehrer DS, Castle DJ. Psychiatric comorbidities and schizophrenia. Schizophr Bull. 2009;35(2):383-402.

16. van Rossum I, Lieb R, Wittchen H-U, van Os J. Affective dysregulation and reality distortion: a 10-year prospective study of their association and clinical relevance. Schizophr Bull. 2011;37(3):561-71.

17. Thewissen V, Bentall RP, Oorschot M, à Campo J, van Lierop T, van Os J, et al. Emotions, self-esteem, and paranoid episodes: an experience sampling study. Br J Clin Psychol. 2011;50(2):178-95.

18. McGorry PD, Nelson B, Goldstone S, Yung AR. Clinical staging: a heuristic and practical strategy for new research and better health and social outcomes for psychotic and related mood disorders. Can J Psychiatry. 2010;55(8):486-97.

19. Scott J, Leboyer M, Hickie I, Berk M, Kapczinski F, Frank E, et al. Clinical staging in psychiatry: a crosscutting model of diagnosis with heuristic and practical value. Br J Psychiatry. 2013;202(4):243-5.

20. Wigman JT, van Os J, Thiery E, Derom C, Collip D, Jacobs N, et al. Psychiatric diagnosis revisited: towards a system of staging and profiling combining nomothetic and idiographic parameters of momentary mental states. PLoS One. 2013;8(3):e59559. 
21. van Os J, Lataster T, Delespaul P, Wichers M, Myin-Germeys I. Evidence that a psychopathology interactome has diagnostic value, predicting clinical needs: an experience sampling study. PLoS One. 2014;9(1):e86c652.

22. Regier DA, Narrow WE, Kuhl EA, Kupfer DJ. The conceptual development of DSM-V. Am J Psychiatry. 2009;166(6):645-50.

23. McGorry $\mathrm{P}$, van Os J. Redeeming diagnosis in psychiatry: timing versus specificity. Lancet. 2013;381(9863):343-5.

24. Kendell R, Jablensky A. Distinguishing between the validity and utility of psychiatric diagnoses. Am J Psychiatry. 2003;160(1):4-12.

25. Van Os J. Are psychiatric diagnoses of psychosis scientific and useful? The case of schizophrenia. J Ment Health. 2010;19(4):305-17.

26. Wichers M. The dynamic nature of depression: a new micro-level perspective of mental disorder that meets current challenges. Psychol Med. 2014;44(7):1349-60.

27. Borsboom D, Cramer AO, Schmittmann VD, Epskamp S, Waldorp LJ. The small world of psychopathology. PLoS One. 2011;6(11):e27407.

28. Kendler K, Zachar P, Craver C. What kinds of things are psychiatric disorders? Psychol Med. 2011;41(6):1143-50.

29. van Os J, Delespaul P, Wigman J, Myin-Germeys I, Wichers M. Beyond DSM and ICD: introducing "precision diagnosis" for psychiatry using momentary assessment technology. World Psychiatry. 2013;12(2):113-7.

30. Myin-Germeys I, Birchwood M, Kwapil T. From environment to therapy in psychosis: a real-world momentary assessment approach. Schizophr Bull. 2011;37(2):244-7.

31. Dichter GS, Felder JN, Petty C, Bizzell J, Ernst M, Smoski MJ. The effects of psychotherapy on neural responses to rewards in major depression. Biol Psychiatry. 2009;66(9):886-97.

32. GeschwindN, Peeters F, Drukker M, van Os J, Wichers M. Mindfulness training increases momentary positive emotions and reward experience in adults vulnerable to depression: a randomized controlled trial. J Consult Clin Psychol. 2011;79(5):618-28.

33. Fredrickson BL, Cohn MA, Coffey KA, Pek J, Finkel SM. Open hearts build lives: positive emotions, induced through loving-kindness meditation, build consequential personal resources. J Pers Soc Psychol. 2008;95(5):1045.

34. Telford C, McCarthy-Jones S, Corcoran R, Rowse G. Experience sampling methodology studies of depression: the state of the art. Psychol Med. 2012;42(6):1119-29.

35. Wichers M, Hartmann JA, Kramer IMA, Lothmann C, Peeters F, van Bemmel L, et al. Translating assessments of the film of daily life into person-tailored feedback interventions in depression. Acta Psychiatr Scand. 2011;123(5):402-3. 


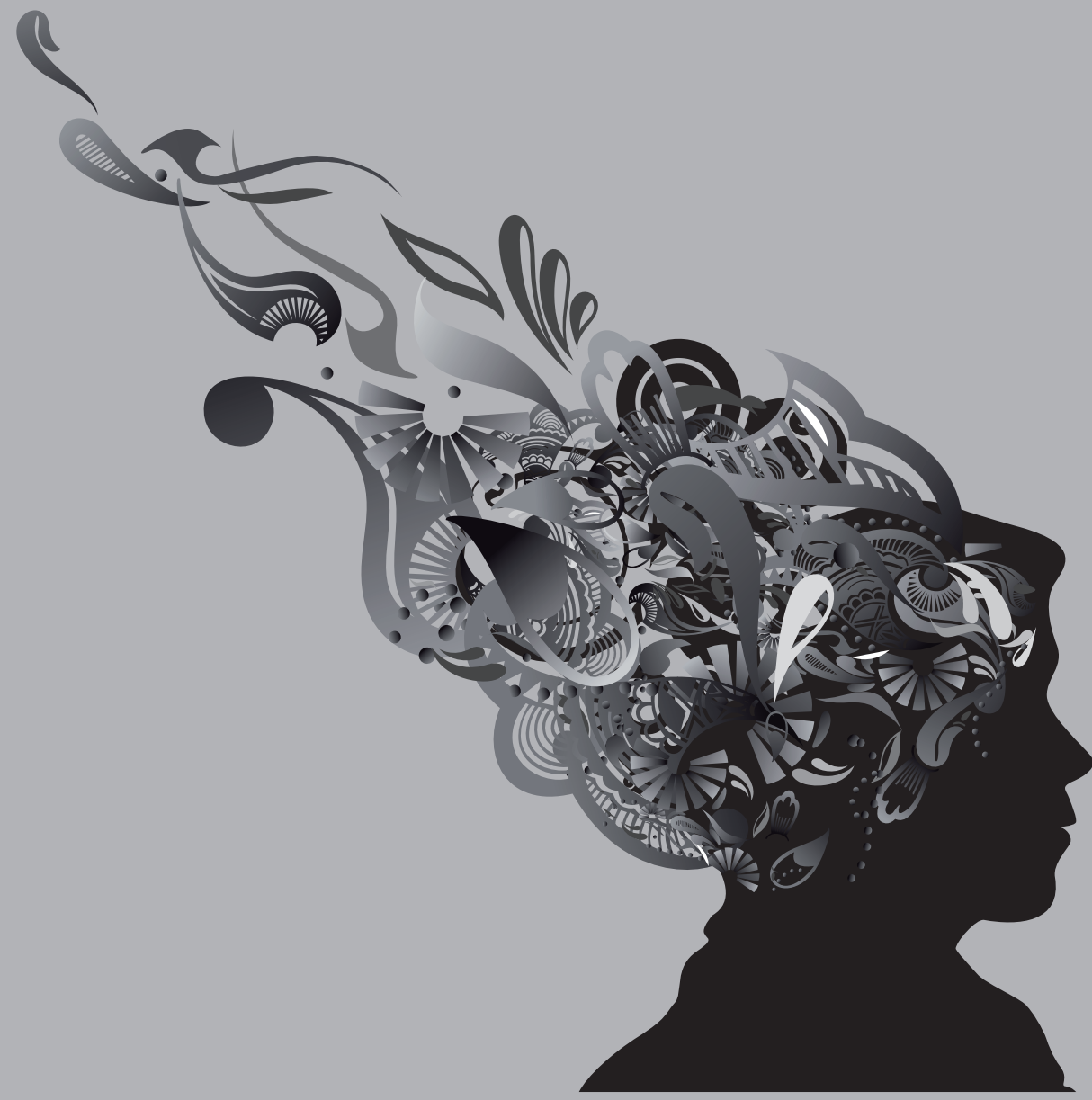




\section{CHAPTER 7}

\section{Valorization, clinical implications}

and directions for future research 


\section{Relevance}

Mental disorders are common $(1,2)$. Symptoms of a mental disorder interfere in the daily life of the individual and may create a substantial burden to the individual and his/her family (1). Due to these symptoms, an individual may be less able or unable to fulfill daily roles and activities. Mental disorders are an important cause of loss of productivity and societal costs $(3,4)$. Better strategies for prevention and treatment are needed (1).

Using the Experience Sampling Method (ESM), in the present thesis a shift was made from the macro-level of symptoms of a mental disorder to the micro-level of emotional experiences and behavioral patterns in order to explore whether this may contribute to a better understanding of mechanisms underlying the development of symptoms of a mental disorder (chapter 2 and 3) and whether it may have a place in treatment of mental disorders (chapter 4 and 5).

\section{Target groups}

The present work has demonstrated that affective symptomatology may contribute to the development of psychotic experiences and symptoms (chapter 2 and 3). This finding is of relevance to mental health professionals: awareness and acknowledgement of the importance of affective symptomatology and its putative role in the development of psychotic experiences and symptoms needs to be taken into account in the development of new effective interventions and (relapse) prevention strategies.

Although the use of ESM in clinical practice is still in its infancy, actual implementation and use may have impact on several levels in mental health care, ranging from the patients, to mental health professionals and possibly the policy makers.

\section{Patients}

Using ESM in clinical practice is a collaborative venture of patient and treating professional $(5,6)$. Patients can become experts in their own emotional dynamics and behavioral patterns, potentially resulting in enhanced empowerment (6). Using ESM may help to refine the diagnostic process (ESM-diagnosis, ESM-D), and may pinpoint to emotional experiences and/or behavioral patterns that are in need for change (ESMmonitoring, ESM-M). Finally, ESM may also be used to deliver real-time, real-world intervention to the patient (ESM-I) (chapter 4, 5 (6-9)). In the present thesis, an ESMbased intervention (ESM-I) was developed for the treatment of depression (chapter 5). 


\section{Mental health care professionals}

As the use of ESM in clinical practice may help to bridge the gap between the professional's office and the patient's daily life, the professional will gain access to the actual emotional dynamics and behavioral patterns of the individual patient. Hence, the professional will be informed in a reliable and ecological valid matter, resulting in a larger window of observation. This may not only result in a better understanding of the problems of the patients but also provides the possibility to deliver an out of the office intervention. The therapeutic setting may thus be enlarged from the professional's office to the daily life of the individual patient, creating a larger window of opportunity for intervention. Nonetheless, a consequence of using ESM in clinical practice is that professionals need to be trained in (i) how to gain access to the ESM data, and (ii) the interpretation and use of the ESM data in the therapeutic patient-professional dyad.

\section{Policy makers}

Give the rising costs of mental health care, new treatment options that are effective and cost-effective are needed and of interest for policymakers. The present endeavor demonstrated the effectiveness and feasibility of an ESM-based intervention complementary to a pharmacological intervention in the treatment of depression (chapter 5). Simons et. al. (unpublished work) examined the cost-effectiveness of this ESM intervention. Preliminary results indicated that using this ESM-intervention complementary to standard pharmacological treatment was indeed cost-effective.

\section{Dissemination and products}

The work in this thesis has been published in scientific peer reviewed journals and was presented at scientific and professional conferences.

The ESM-intervention (ESM-I) device (the 'Psymate') that was used in the RCT in chapter 5 is currently been used in other studies. As technological advances progress rapidly, a PsyMate app has been developed which is downloadable (free of charge) from the Google Playstore and the Apple Appstore. This app is suitable for iOS and android systems; it can be installed on mobile phones and tablets. On a corresponding website, individuals can look at the results of their ESM assessments. Thanks to this app, ESM-I will rapidly be disseminated into a broader public, both mental health workers as the general public. Several mental health institutions have already shown their interest in the PsyMate app and are willing to use ESM-I in treatment of mental disorders. 


\section{Innovation}

To our knowledge, the randomized controlled trial presented in this dissertation is the first RCT using ESM as a novel therapeutic intervention (ESM-I) in depressed patients with a view to improve personalized treatment (chapter 5). Thanks to the recent development and availability of digital ESM devices and web-based applications, real-life data on emotional dynamics becomes easily available to patients and health care professionals. This may help to bridge the gap between the therapist office and an individual's daily life, by refining the diagnostic process (ESM-D), creating the possibility to monitor psychiatric symptomatology in the flow of daily life (ESM-M), and the opportunity to provide an out-of-the-office ESM intervention (ESM-I) to the individual (chapter 4, 5 (6-9)). ESM thus provides the opportunity to 'personalize' the diagnostic process and treatment possibly resulting in an enhanced treatment effect $(8,10-13)$. It can be easily implemented in standard mental health care and does not require much additional investment of clinicians $(7,10)$. In sum, ESM opens a whole new venue on psychiatric diagnosis and intervention possibilities.

\section{Clinical implementation: possibilities, challenges and concerns}

As technology rapidly progresses, new opportunities to collect daily life data are developed. There are a growing number of mobile phone or tablet applications and devices (e.g. wearables) directed to gather data and monitor both physiological and psychological parameters of (ill) health (see for example www.smarthealth.nl). Continuous ambulatory assessments of objective physiological parameters such as blood pressure, heart rate, blood glucose, and movement are already used in somatic medicine. Combining the assessment of objective physiological parameters with ESM assessments of for example emotional experiences, psychological health, habits, dietary intake, medication adherence and behavioral patterns over time, would result in an increasingly detailed biopsychosocial film of the daily life of the individual. Behavioral and psychological interventions can be delivered in real-life at the moment itself when one is for example confronted with a stressor or experiences an anomalous event after which the effect of the intervention can be monitored.

The present work indicates that ESM can make an important contribution to clinical practice. Yet, actual implementation in mental health care is still in its early years and needs to be further developed and examined. Earlier work pointed to the risk of withdrawal of the health care professional and disengagement of the patient (14). The present endeavor, in contrast, showed that long-term monitoring (an 8 week period) was considered feasible by mild to severe depressed patients, and that completion rates 
of the ESM assessments were high (chapter 5).

Given the almost endless opportunities in data gathering, ethical issues with respect to information safety and data ownership arise. Notwithstanding these (future) challenges, the present work indicates that ESM may constitute a new viable approach to improve personalized mental health care.

\section{Directions for future research}

By integrating the findings and insights of the present thesis, several suggestions for future research can be made. In the battle against psychosis, attention for the depression continuum is important. As was demonstrated on the micro-level of negative affective experiences and macro-level of depressive symptomatology, the depression continuum is associated with the onset of paranoia and psychotic symptomatology. This association needs to be examined in greater detail in future research. Using a network approach, the role of affective symptomatology in the pathway to psychosis may be further explored.

The present work showed that ESM has the potential to contribute to clinical practice. We made a first attempt to develop an ESM-derived feedback module focused on PA. Although this intervention resulted in a reduction of depressive symptomatology, the exact mechanism by which it impacted on depressive symptomatology needs to be elucidated in future work. On a more practical level, the precise content of the PA focused feedback is an important theme in the development of new ESM interventions, as well as potentially elaborating the feedback with biological assessments of for example movement and heart rate.

An exciting application of ESM in the diagnostic process would consist of complementing and refining current diagnostic methods by the construction of a personalized micro-level network of emotional experiences, behavior and symptoms $(5,6,9)$. By transforming implicit real-life dynamic patterns to explicit, visualized and quantifiable configurations, specific dysfunctional patterns in the daily life of the individual patient that are in need for change can be identified. Of specific interest is the identification of 'positive nodes' (defined as nodes that reflect a protective factor in the development of psychopathology) in such a network (15). A positive node may be an essential link in the switch from mental ill health to mental health. Given the well-known contribution of positive affect to mental health $(16,17)$, it is attractive to speculate that positive affect may be such a positive node.

Zooming into the micro-level of experience thus resulted in new insights in mechanisms underlying the development of psychopathology that could also contribute to the development of effective interventions. Therefore, it may help to bridge the gap between science and clinical practice. 


\section{References}

1. Wittchen HU, Jacobi F, Rehm J, Gustavsson A, Svensson M, Jönsson B, et al. The size and burden of mental disorders and other disorders of the brain in Europe 2010. Eur Neuropsychopharmacol. 2011;21(9):65579.

2. Bijl RV, Ravelli A, van Zessen G. Prevalence of psychiatric disorder in the general population: results of the Netherlands Mental Health Survey and Incidence Study (NEMESIS). Soc Psychiatry Psychiatr Epidemiol. 1998;33(12):587-95.

3. de Graaf R, Tuithof M, van Dorsselaer S, ten Have M. Comparing the effects on work performance of mental and physical disorders. Soc Psychiatry Psychiatr Epidemiol. 2012; 47(11):1873-83.

4. Gustavsson A, Svensson M, Jacobi F, Allgulander C, Alonso J, Beghi E, et al. Cost of disorders of the brain in Europe 2010. Eur Neuropsychopharmacol. 2011;21(10):718-79.

5. van Os J, Lataster T, Delespaul P, Wichers M, Myin-Germeys I. Evidence that a psychopathology interactome has diagnostic value, predicting clinical needs: an experience sampling study. PLoS One. 2014;9(1):e86652.

6. van Os J, Delespaul P, Wigman J, Myin-Germeys I, Wichers M. Beyond DSM and ICD: introducing "precision diagnosis" for psychiatry using momentary assessment technology. World Psychiatry. 2013;12(2):113-7.

7. Myin-Germeys I, Birchwood M, Kwapil T. From environment to therapy in psychosis: a real-world momentary assessment approach. Schizophr Bull. 2011;37(2):244-7.

8. Wichers M, Hartmann JA, Kramer IMA, Lothmann C, Peeters F, van Bemmel L, et al. Translating assessments of the film of daily life into person-tailored feedback interventions in depression. Acta Psychiatr Scand. 2011;123(5):402-3.

9. Wichers M. The dynamic nature of depression: a new micro-level perspective of mental disorder that meets current challenges. Psychol Med. 2014;44(7):1349-60.

10. Wichers M, Simons CJP, Kramer IMA, Hartmann JA, Lothmann C, Myin-Germeys I, et al. Momentary assessment technology as a tool to help patients with depression help themselves. Acta Psychiatr Scand. 2011;124(4):262-72.

11. Trull TJ, Ebner-Priemer UW. Using experience sampling methods/ecological momentary assessment (ESM/EMA) in clinical assessment and clinical research: introduction to the special section. Psychol Assessment. 2009;21(4):457-62.

12. Trull TJ, Ebner-Priemer U. Ambulatory assessment. Annu. Rev Clin Psychol. 2013;9:151-76.

13. Heron KE, Smyth JM. Ecological momentary interventions: incorporating mobile technology into psychosocial and health behaviour treatments. Br J Health Psychol. 2010;15(1):1-39.

14. van der Feltz-Cornelis CM, van Os J, Knappe S, Schumann G, Vieta E, Wittchen HU, et al. Towards Horizon 2020: challenges and advances for clinical mental health research - outcome of an expert survey. Neuropsychiatr Dis Treat. 2014;10:157-68.

15. Borsboom D, Cramer AO, Schmittmann VD, Epskamp S, Waldorp LJ. The small world of psychopathology. PLoS One. 2011;6(11):e27407.

16. Dunn BD. Helping depressed clients reconnect to positive emotion experience: current insights and future directions. Clin Psychol Psychother. 2012;19:326-40.

17. Garland EL, Fredrickson B, Kring AM, Johnson DP, Meyer PS, Penn DL. Upward spirals of positive emotions counter downward spirals of negativity: insights from the broaden-and-build theory and affective neuroscience on the treatment of emotion dysfunctions and deficits in psychopathology. Clin Psychol Rev. 2010;30:849-64. 


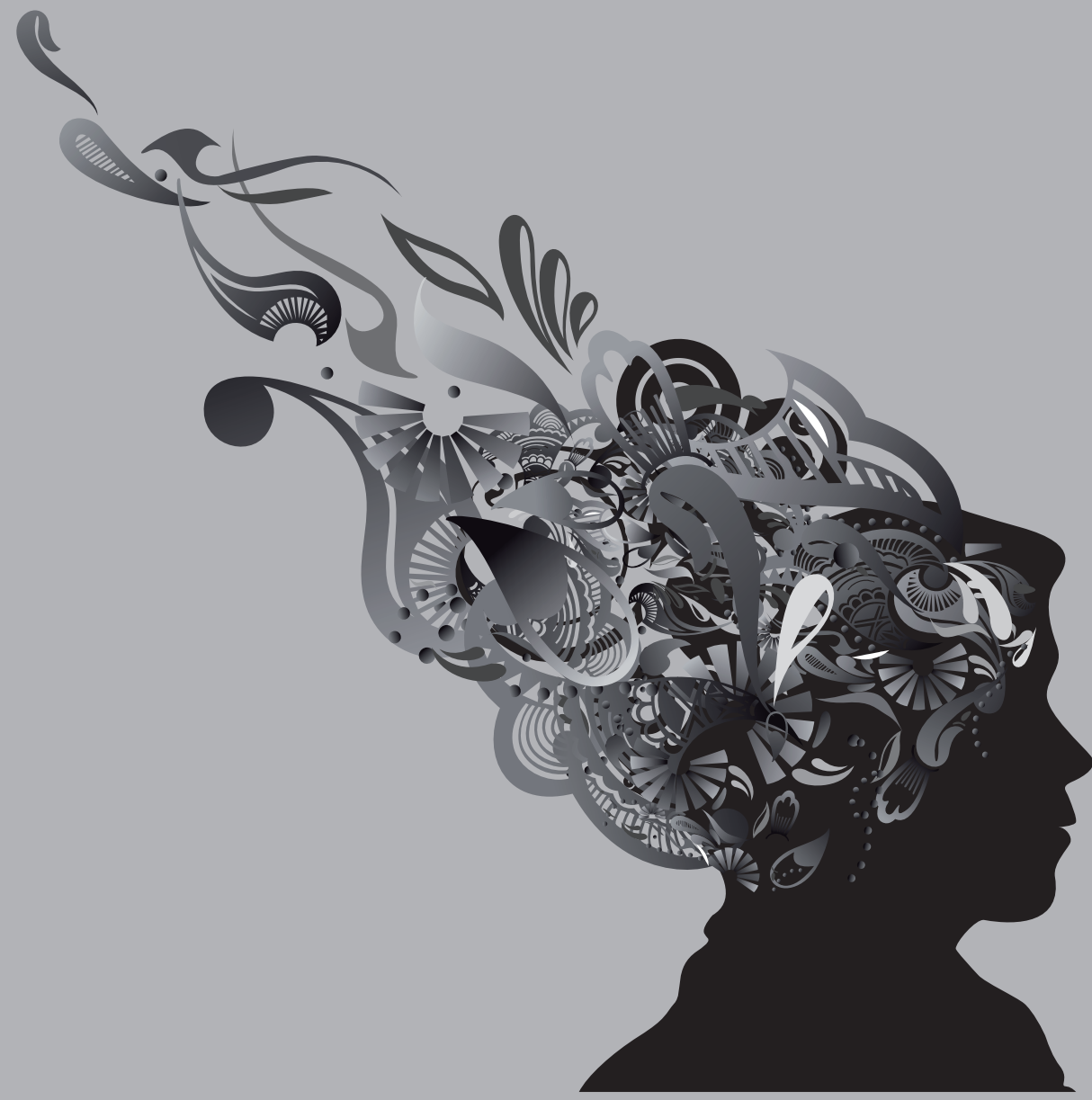


EPILOGUE 


\section{Summary}

This thesis focuses on the use of ambulatory assessment in mental health care. Using ambulatory assessment or the Experience Sampling Method (ESM), one's current context, mood, thoughts, and symptoms can be assessed in the flow of one's daily life. It is a structured diary technique that involves repetitive, prospective assessments that results in a fine-grained, high resolution ('micro-level') film of daily life emotional experiences and behavioral patterns embedded in their context. Using ESM, emotional dynamics within a person, over time and in different contexts can be examined. In the present thesis, it was examined whether a shift from the macro-level of symptomatology to the micro-level of emotional experiences would result in a better understanding of mechanisms underlying the development of symptoms of mental disorders (chapter 2 and 3). The second part of this thesis continues on the micro-level of emotional experiences and explores whether this may contribute to the treatment of mental disorders (chapter 4 and 5).

In chapter 1, a general introduction to the two mental disorders that this thesis focusses on, depression and psychosis, is provided. Affective dynamics seem to play an important role in the development of both depression and psychosis. The affective pathway to psychosis and subsequently the Experience Sampling Method are introduced. Chapter 1 ends with an overview of the aims and outline of this thesis..

Chapter 2 starts at the macro-level of symptomatology. Symptoms of depression and psychosis tend to cluster within individuals and families. Genes for depression may act by making individuals more sensitive to childhood trauma. As childhood adversity has been identified as a risk factor for the development of adult psychosis, the aim in chapter 2 was to examine whether the association between childhood adversity and psychotic symptoms was moderated by a genetic liability for depression. Furthermore, putative mechanisms underlying this moderation were studied; it was examined to what degree an increase in stress-sensitivity or depressive symptoms themselves occasioned the moderating effect. In a female twin sample, it was demonstrated that the effect of childhood adversity on follow-up psychotic symptoms (measured by the Symptom Checklist-90 Revised (SCL-90-R) and the Community Assessment of Psychic Experiences (CAPE)) was significantly moderated by genetic vulnerability for depression. The moderation by genetic liability was mediated by depressive experience, but not by stress sensitivity. A genetic liability for depression may potentiate the 
pathway from childhood adversity to psychotic symptoms. Dysfunctional emotional processing of anomalous experiences associated with childhood trauma may be an underlying mechanism.

In chapter 3, a shift to the micro-level of daily life emotional dynamics was made to examine whether and how exactly affective experiences impact on the risk for psychosis. Using ESM, moment-to-moment dynamics between negative affect (NA) and paranoia were examined in the same general population subject sample as used in chapter 2 . Results indicated that moments of NA increase resulted in an increase in paranoia over 180 subsequent minutes. The shift to the micro-level of hour-by-hour tracking of emotional dynamics made it also possible to examine how shared risk factors for depression and psychosis impacted on the dynamics between NA and subsequent paranoid experiences. Both stress sensitivity and depressive symptoms impacted on the transfer of NA to paranoia. While stress sensitivity boosted paranoia during the moment of NA increase, depressive symptoms increased the persistence of momentto-moment paranoid experiences. Depressive symptoms thus increased the time that paranoid feelings remained present over the day. The shift to the micro-level of daily life emotional dynamics thus not only revealed temporal NA-paranoia dynamics but also suggested how risk factors changed the micro-level of hour-to-hour interplay between NA and paranoia by increasing the amplitude or persistence of paranoia experiences following NA boosts. These subtle changes in repetitive everyday emotional dynamics were found to be associated with follow-up SCL-90-R and CAPE psychotic symptoms. These psychotic symptoms, when persistent, may eventually constitute risk for later clinical symptoms, help-seeking behavior, and finally the development of a clinical disorder. Examination of micro-level momentary experiences may thus yield new insights into the mechanism underlying co-occurrence of altered mood states and psychosis. Knowledge of underlying mechanisms is required in order to determine source and place where intervention and remediation should occur.

Affective dynamics play a role in the risk for development of mental disorders. Using ESM, changes in affective experiences (NA and positive affect (PA)) can be revealed. Depression, among other characteristics, is characterized by an increase in NA, a decrease in PA, high rates of relapse and frequent residual symptoms. Therefore, new strategies to increase treatment effectiveness are required. In chapter $\mathbf{4}$, a literature review was conducted to examine how electronic Experience Sampling Methodology may contribute to the assessment and intervention in depression. Experience sampling assessments are able to reveal subtle, small but repetitive and relevant patterns of emotional experiences. An experience sampling tool may reveal manageable pieces of 
daily life behavior contributing to depressive experiences that patients can influence. The use of this explicit knowledge of daily life experiences and behavioral patterns is understudied with regard to its potential contribution to the diagnostic process, monitoring of treatment effect, and intervention possibilities in depressed patients. The clinical application of ESM may stimulate a shift from passive consumption of treatment to an active role of patients in their recovery and increased ownership. The precise, prospective and fine-grained information that ESM provides may contribute to clinical practice in various way. Future studies should examine the clinical impact of its use and the feasibility of its implementation in mental health care.

An example of the use of ESM in mental health care could be the assessment and monitoring of affective experiences of patients. In depression, the ability to experience daily life PA predicts recovery and reduces relapse rates. Interventions based on the experience sampling method (ESM-I) are ideally suited to provide insight in personal, contextualized patterns of PA. In chapter 5, it was examined whether ESM-derived feedback on personalized patterns of PA is feasible and useful to patients as add-on to pharmacotherapy, and results in a reduction of depressive symptomatology. Depressed outpatients $(n=102)$ receiving pharmacological treatment participated in a randomized controlled trial with three arms: an experimental group receiving add-on ESM-derived feedback, a pseudo-experimental group participating in ESM but receiving no feedback, and a control group. The experimental group participated in an ESM procedure (three days per week over a 6-week period) using a palmtop ('PsyMate'). This group received weekly standardized feedback on personalized patterns of PA. Hamilton Depression Rating Scale-17 (HDRS) and Inventory of Depressive Symptoms (IDS) scores were obtained before and after the intervention. During a 6-month follow-up period, five HDRS and IDS assessments were completed. Add-on ESM-derived feedback resulted in a significant and clinically relevant stronger decrease in HDRS score relative to the control group. Compared to the pseudo-experimental group, a clinically relevant decrease in HDRS score was apparent at 6 months. Self-reported depressive complaints (IDS) yielded the same pattern over time. The use of ESM-I was deemed acceptable and the provided feedback easy to understand. Patients attempted to apply suggestions from ESM-derived feedback to daily life. These data suggest that the efficacy of traditional passive pharmacological approach to treatment of major depression can be enhanced by using person-tailored daily life information regarding PA.

In chapter 6, the main findings of this thesis are discussed. The results of chapters 2 and 3 were integrated in a new model that demonstrated how risk factors for psychosis may impact on the micro-level of moment-to-moment emotional experience that may 
over time result in clinical psychotic disorder. Furthermore, the contribution of ESM in the search for mechanisms underlying psychopathology is discussed as well as its potential contribution to the treatment of mental disorders such as depression (chapter 4 and 5). Chapter 7 closes with valorization, clinical implications and suggestions for future research. 


\section{Samenvatting}

In dit proefschrift wordt het gebruik van ambulante metingen in de geestelijke gezondheidszorg onderzocht. Door middel van ambulante metingen oftewel de Experience Sampling Methode (ESM) kunnen context, stemming, gedachtes en symptomen in de loop van iemands dagelijks leven gemeten worden. Het is een gestructureerde dagboekmethode bestaande uit opeenvolgende metingen die zeer gedetailleerde, hoge resolutie informatie ('microniveau') over alledaagse emotionele ervaringen, gedragspatronen en bijbehorende context oplevert. Met behulp van ESM kan de dynamiek tussen verschillende emoties van een persoon, optredende in de loop van de tijd en in verschillende contexten, onderzocht worden. In dit proefschrift werd onderzocht of een switch van het niveau van symptomen (macroniveau) naar het niveau van emotionele ervaringen (microniveau) kan leiden tot een beter begrip van mechanismen die aan het ontstaan van psychiatrische aandoeningen ten grondslag liggen (hoofdstuk 2 en 3). In het tweede gedeelte van dit proefschrift werd onderzocht of kennis op het microniveau van emotionele ervaringen kan bijdragen aan de behandeling van psychiatrische aandoeningen (hoofdstuk 4 en 5).

In hoofdstuk 1 wordt een algemene inleiding over twee in dit proefschrift centraal staande psychiatrische aandoeningen gegeven, depressie en psychose. In de ontwikkeling van zowel depressie als psychose lijken affectieve dynamieken een belangrijke rol te spelen. Het affectieve pad naar psychose en vervolgens de Experience Sampling Methode wordt geïntroduceerd. Hoofdstuk 1 eindigt met een beschrijving van de doelen van dit proefschrift waarbij een overzicht van de onderwerpen van de verschillende hoofdstukken gegeven wordt.

Hoofdstuk 2 begint op het macroniveau van symptomatologie. Symptomen van depressie en psychose komen vaak samen binnen dezelfde persoon maar ook binnen een en dezelfde familie voor. Genen die geassocieerd zijn met het ontwikkelen van een depressie zouden hun invloed kunnen uitoefenen door individuen kwetsbaarder te maken voor jeugdtrauma. Jeugdtrauma blijkt een risicofactor voor de ontwikkeling van psychose te zijn. Daarom werd in hoofdstuk 2 onderzocht of de associatie tussen jeugdtrauma en het ontwikkelen van psychotische symptomen beïnvloed werd door een genetische kwetsbaarheid voor depressie. Vervolgens werden mogelijke hieraan ten grondslag liggende mechanismen onderzocht; onderzocht werd in welke mate een toename in stressgevoeligheid of daadwerkelijke depressieve symptomen aan dit verband ten grondslag lagen. In een groep vrouwelijke tweelingen werd aangetoond 
dat het effect van jeugdtrauma op toekomstige psychotische symptomen (gemeten met de Symptom Checklist-90 Revised (SCL-90-R) en de Community Assessment for Psychic Experiences (CAPE)) significant beïnvloed werd door een genetische kwetsbaarheid voor depressie. Aan deze beïnvloeding lag niet stressgevoeligheid, maar wel de aanwezigheid van depressieve symptomen ten grondslag. Een genetische kwetsbaarheid voor depressie kan dus leiden tot een versterking van de relatie tussen jeugdtrauma en het ontstaan van psychotische symptomen. Een afwijkende emotionele verwerking van abnormale met jeugdtrauma geassocieerde ervaringen is hierbij een mogelijk onderliggend mechanisme.

In hoofdstuk 3 werd een switch van het macroniveau van symptomatologie naar het microniveau van emotionele ervaringen gemaakt. Onderzocht werd of affectieve ervaringen het risico op de ontwikkeling van psychose beïnvloeden en hoe dat gebeurt. Met behulp van ESM werd van moment tot moment de relatie tussen negatief affect (NA) en wantrouwen onderzocht in de steekproef van vrouwelijke tweelingen die ook in hoofdstuk 2 werd gebruikt. Een stijging in NA bleek gevolgd te worden door een periode met toegenomen wantrouwen. Deze stijging in wantrouwen was tot 180 minuten later significant. Het van uur-tot-uur volgen van de dynamiek tussen NA en wantrouwen maakte het mogelijk om te onderzoeken hoe gedeelde risicofactoren voor depressie en psychose deze dynamiek beïnvloedden. Zowel stress gevoeligheid als depressieve symptomen beïnvloedden het ontstaan van wantrouwen na een stijging in NA. Daar waar stressgevoeligheid tot een toename van wantrouwen tijdens de stijging in NA leidde, waren het de depressieve symptomen die tot het aanhouden (persistentie) van de momenten met wantrouwen leidden. Depressieve symptomen leidden dus tot een langere aanwezigheid van wantrouwen in het dagelijks leven. De switch naar het microniveau van alledaagse emotionele dynamieken bracht dus niet alleen tijdelijke veranderingen tussen NA en wantrouwen in kaart, maar liet ook zien hoe verschillende risicofactoren deze tijdelijke dynamiek beïnvloedden door enerzijds een toename van de amplitude of anderzijds het persisteren van wantrouwen die optreedt na een stijging in NA. Deze subtiele veranderingen in alledaagse, zich herhalende emotionele dynamieken waren geassocieerd met follow-up SCL-90-R en CAPE psychotische symptomen. Deze psychotische symptomen kunnen als ze persisteren een risico voor de ontwikkeling voor klinische psychotische symptomen, hulpzoekend gedrag en uiteindelijk de ontwikkeling van een klinische stoornis vormen. Onderzoek op het microniveau van emotionele ervaringen kan dus tot nieuwe inzichten in mechanismen die ten grondslag liggen aan het gelijktijdig optreden van affectieve veranderingen en psychose leiden. Kennis van onderliggende (micro)mechanismen is belangrijk om te bepalen op welk moment welke (herstelgerichte) interventie dient plaats te vinden. 
Affectieve dynamieken spelen een rol bij het risico op ontwikkeling van mentale stoornissen. Met behulp van de Experience Sampling Methode kunnen dynamieken tussen affectieve ervaringen (zoals NA en positief affect (PA)) in kaart gebracht worden. Bij depressie is er onder andere sprake van een toename in NA, een afname in PA, een hoog terugvalpercentage en het frequent optreden van restsymptomen. Het is belangrijk om nieuwe manieren ter vergroting van de effectiviteit van behandeling van depressie te vinden. In hoofdstuk 4 werd een literatuurstudie verricht waarin onderzocht werd hoe elektronische Experience Sampling metingen zouden kunnen bijdragen aan het vaststellen van en interveniëren bij een depressie. Experience Sampling metingen maken het mogelijk om subtiele, kleine doch herhaaldelijk optredende, relevante patronen in emotionele ervaringen bloot te leggen. Een Experience Sampling apparaat zou hanteerbare door patiënten te beïnvloeden stukjes alledaags gedrag dat bijdraagt aan depressieve ervaringen bloot kunnen leggen. De bijdrage die het gebruik van deze expliciete kennis over alledaagse ervaringen en alledaags gedrag aan het diagnostische proces, opvolging van behandeleffect en mogelijkheden tot interventie bij depressieve patiënten kan leveren, is vooralsnog een onderbelicht terrein. Klinisch gebruik van ESM zou bij de patiënt kunnen leiden tot een switch van passieve zorgconsumptie naar het hebben van een actieve rol en 'eigenaarschap' in het herstelproces. Gedetailleerde, hoge resolutie, prospectieve ESM data zou op een aantal manieren aan de klinische praktijk kunnen bijdragen. Toekomstig onderzoek naar de klinische impact van het gebruik van ESM en de haalbaarheid hiervan in de geestelijke gezondheidzorg is nodig.

Een van de toepassingen van ESM in de geestelijke gezondheidszorg zou het in kaart brengen en monitoren van affectieve ervaringen van patiënten kunnen zijn. Het vermogen om positief affect (PA) in het dagelijks leven te ervaren voorspelt het herstel van depressie en vermindert de kans op een terugval. Interventies die gebaseerd zijn op de Experience sampling Methode (ESM-I) hebben de mogelijkheid om inzicht in persoonlijke, context gebonden patronen in PA te verschaffen. In hoofdstuk $\mathbf{5}$ werd onderzocht of op ESM gebaseerde feedback over persoonlijke patronen in PA haalbaar en nuttig is voor patiënten en of dit toegevoegd aan farmacotherapie leidt tot een afname in depressieve klachten. Ambulante depressieve patiënten $(n=102)$ die antidepressieve medicatie gebruikten, namen deel aan een gerandomiseerde gecontroleerde trial met drie armen: een experimentele groep die op ESM gebaseerde feedback ontving, een pseudo-experimentele groep die deelnam aan de ESM metingen maar geen feedback ontving, en een controle groep. De experimentele groep participeerde in de ESM procedure (3 dagen per week gedurende 6 weken), gebruikmakende van een digitaal ESM apparaat ('PsyMate'). Deze groep ontving wekelijks gestandaardiseerde feedback gericht op het ervaren van PA in de alledaagse context. De Hamilton Depression Rating 
Scale-17 (HDRS) en de zelfinvullijst depressieve symptomen (Inventory of Depressive Symptoms (IDS)) werden voor en na de interventie afgenomen. Gedurende een followup periode van 6 maanden werden 5 HDRS en IDS metingen afgenomen. Het ontvangen van toegevoegde, op ESM gebaseerde feedback leidde in vergelijking met de controle groep tot een significante en klinisch relevante afname in HDRS score. In vergelijking met de pseudo-experimentele groep was er na 6 maanden sprake van een klinisch relevante afname in HDRS score. Zelfgerapporteerde depressieve symptomen (IDS) lieten een vergelijkbaar patroon over de tijd zien. Het gebruik van ESM-I werd door patiënten als acceptabel ervaren en de verstrekte feedback was gemakkelijk te begrijpen. Patiënten probeerden suggesties die voortvloeiden uit de op ESM-gebaseerde feedback toe te passen in hun dagelijks leven. Deze bevindingen suggereren dat de effectiviteit van reguliere passieve medicamenteuze behandeling van depressie verhoogd kan worden door gebruik te maken van gepersonaliseerde informatie over PA uit het alledaagse leven van de patiënt.

In hoofdstuk 6 worden de belangrijkste bevindingen van dit proefschrift besproken. De resultaten van hoofdstuk 2 en 3 werden geïntegreerd in een nieuw model dat liet zien hoe risicofactoren voor psychose invloed kunnen hebben op het microniveau van momentane emotionele ervaringen welke na verloop van tijd kunnen leiden tot een klinische psychotische stoornis. Vervolgens werd ingegaan op de bijdrage van ESM in het onderzoek naar micro-mechanismen in het ontstaan van psychopathologie en de mogelijke bijdrage van ESM aan de behandeling van mentale stoornissen zoals depressie (hoofdstuk 4 en 5). Tot slot werden in hoofdstuk 7 de valorisatie, klinische implicaties en suggesties voor toekomstig onderzoek besproken. 


\section{Dankwoord}

De laatste punt is gezet; het proces van loslaten kan beginnen. Hieraan is de realisatie verbonden dat dit meerjaren project niet mogelijk zou zijn geweest zonder de steun en hulp van een grote groep mensen. Hiervoor mijn grote dankbaarheid.

Het begon allemaal in de eindfase van mijn opleiding tot psychiater, toen ik bij mijn opleider Dr. Lex van Bemmel binnenliep met de vraag of het mogelijk was om mijn keuzejaar aan onderzoek te besteden. Zijn enthousiaste reactie leidde tot een kennismaking met drie zeer getalenteerde mensen, mijn promotieteam. Allereerst gaat mijn dank uit naar mijn promotor, Prof. dr. Jim van Os. Jim, dank voor de mogelijkheid onderzoek onder jouw supervisie en begeleiding uit te voeren, de stimulerende en inspirerende reacties op mijn werk. De snelheid hiervan is werkelijk legendarisch. Het was een groot voorrecht.

Daarnaast gaat een grote dank uit naar mijn beide copromotores, Dr. Marieke Wichers en Dr. Claudia Simons. Marieke, heel erg bedankt voor je inspirerende en deskundige begeleiding. Jouw optimisme is een grote kracht en stimulans. Claudia, jij verzorgde de dagelijkse begeleiding. Ik heb me altijd zeer welkom gevoeld om met vragen, hoe klein dan ook bij je binnen te lopen. Jij maakte altijd tijd voor me vrij. Jim, Marieke, en Claudia: dank je wel!

Lex van Bemmel, als jij mij niet in contact had gebracht met het promotieteam was dit boekje niet geschreven. Dank voor jouw bemiddeling. Ook bedankt voor je bijdrage aan het REMOD oftewel 'Emotionele Veerkracht bij Depressie' project in je rol als opleider en praktiserend psychiater.

Mijn dank gaat verder uit naar de coauteurs van de papers die ik geschreven heb. Bedankt voor jullie bijdrage. Verder wil ik Prof. dr. Catharine Derom en Prof. dr. Evert Thiery bedanken voor het gebruik van het Oost-Vlaams tweelingregister. Hoofdstuk 2 en 3 uit dit proefschrift zijn hierop gebaseerd.

Vervolgens wil ik graag de leden van mijn leescommissie en corona bedanken. Bedankt voor jullie tijd en moeite om mijn proefschrift door te nemen.

Grote dank gaat uit naar mijn collega's van de GGzE die op directe en indirecte wijze betrokken waren bij de totstandkoming van dit proefschrift. Allereerst, Kees Spitters, bedankt dat ik een aantal jaar als psychiater aan de afdeling Onderzoek en Ontwikkeling verbonden kon zijn om dit project te doen.

In het bijzonder wil ik mijn collega's van de crisisdienst bedanken voor hun niet aflatende steun en interesse in mijn onderzoek. Barbara en Sander, bedankt voor jullie flexibiliteit en steun in voor mij drukke tijden. De mogelijkheid een aantal maal een 
aantal weken een 'research holiday' in te plannen, heeft gemaakt dat ik de snelheid erin kon houden en in die periodes echt 'meters kon maken'. Tevens gaat mijn dank uit naar mijn 'AIOS-cohort': Joris, Janneke en Carla. Dierbare collega's met wie ik samen de opleiding tot psychiater doorlopen heb en wie het wel en wee van mijn promotie-traject vanaf de eerste dag gevolgd hebben. Dank voor jullie steun en interesse!

Verder gaat mijn dank uit naar alle collega's van de GGzE die door hun inzet het REMOD-project mede hebben mogelijk gemaakt. Zonder jullie inzet zouden we niet genoeg deelnemers hebben gehad.

Wendy, Truda, Karin en Ron: heel erg bedankt voor jullie hulp in het coördineren van de REMOD-studie en de geboden ondersteuning bij de PsyMate en REMODdataperikelen.

Uiteraard wil ik de rest van het REMOD-team voor hun inzet bedanken. Mijn speciale dank gaat uit naar Jessica. Heel erg bedankt voor je inzet, maar vooral ook voor je steun, betrokkenheid en de vele fijne gesprekken die we gehad hebben. Jij was op de GGzE mijn REMOD-maatje; we hebben samen veel eindeloos-lijkende REMOD-perikelen overleefd. Claudia Menne, Petra, en alle mensen die hebben geholpen in het verzamelen van de REMOD-data: Karina, Karlijn en Esther. Dank je wel.

Natuurlijk wil ik ook mijn paranimfen, Evert en Johan, bedanken. Evert: mijn broer, een niet ophoudende bron van relativering en ironie. Jij hebt geholpen om perspectief te bewaren. Johan: jouw vriendschap van meer dan 25 jaar, verschillende levensfases overstrijkend is voor mij erg belangrijk en steunend geweest bij de uitdagingen en beslissingen die onvermijdelijk op mijn levenspad zijn verschenen.

Nadrukkelijk wil ik ook mijn ouders en schoonouders bedanken, zonder wiens steun in woord en daad ik niet zo ver was gekomen.

Tot slot, Martijn, mijn lieve partner: ik weet dat jij misschien nog wel blijer bent dan ik dat dit project afgerond is. Bedankt voor je steun, flexibiliteit en onbegrensd geloof in mij.

Floris, Maurits, en het kleintje dat in mij groeit: dit proefschrift is aan jullie opgedragen. 


\section{Curriculum Vitae}

Ingrid Kramer werd op 12 april 1973 in Sittard geboren. Na afronding van het VWO examen (1991) is zij psychologie (afgerond in 1996) en vervolgens geneeskunde (afgerond in 2003) aan de Radboud Universiteit te Nijmegen gaan studeren.

In 2004 begon zij binnen de GGzE aan de opleiding tot psychiater. Deze heeft zij met een onderzoeksstage afgerond, hetgeen in een promotietraject binnen de afdeling Psychiatrie en Psychologie van de School for Mental Health and Neuroscience, Universiteit Maastricht resulteerde. Sinds januari 2010 werkt zij als psychiater bij de GGzE en heeft zij patiëntenzorg met onderzoek gecombineerd. 


\section{List of Publications}

\section{Journal articles}

Kramer, I.*, Simons, C.J.P*, Hartmann, J.A., Menne-Lothmann, C., Viechtbauer, W., Peeters, F., et al. A therapeutic application of the experience sampling method in the treatment of depression: A randomized controlled trial. World Psychiatry 2014, 13, 6877. *contributed equally

Kramer, I. De experience-samplingmethode als toegevoegde behandeling van depressie: een RCT. Tijdschrift voor Psychiatrie 2014, 56, 414-415.

Kramer, I., Simons, C.J.P., Wigman, J.T.W., Collip, D., Jacobs, N., Derom, C., et al. Timelagged moment-to-moment interplay between negative affect and paranoia: New insights in the affective pathway to psychosis. Schizophrenia Bulletin 2014, 40, 278-286.

Kramer, I. Negatief affect en wantrouwen in het dagelijks leven. Tijdschrift voor Psychiatrie 2013, 55, 382-383.

Kramer, I.M.A., Simons, C.J.P., Myin-Germeys, I., Jacobs, N., Derom, C., Thiery, E., et al. Evidence that genes for depression impact on the pathway from trauma to psychoticlike symptoms by occasioning emotional dysregulation. Psychological Medicine 2012, 42, 283-294.

Wichers, M, Simons, C.J.P.* , Kramer, I.M.A.* , Hartmann, J.A., Lothmann C., Myin-Germeys, I., et al. Momentary assessment technology as a tool to help patients with depression help themselves. Acta Psychiatrica Scandinavica 2011, 124, 262-272. *contributed equally

Wichers M., Hartmann J.A., Kramer I.M.A., Lothmann C., Peeters F., Van Bemmel, L., Myin-Germeys, I., Delespaul, Ph., Van Os, J., Simons, C.J.P. Translating assessments of the film of daily life into person-tailored feedback interventions in depression. Acta Psychiatrica Scandinavica 2011, 123, 402-403. 


\section{Presentations}

Poster presentation at the $5^{\text {th }}$ Schizofreniecongres: 'het alledaagse ongewoon' in Zwolle, the Netherlands, November 2009. Genetic liability for depression facilitates the pathway from childhood adversity to psychosis.

Poster presentation at the $18^{\text {th }}$ World Congress on Psychiatric Genetics in Athens, Greece, October 2010. Unravelling the association between reality distortion and affective dysregulation using momentary assessment technology.

Poster presentation at the 7th International Conference on Early Psychosis (IEPA) in Amsterdam, the Netherlands, November 2010. Unravelling the association between reality distortion and affective dysregulation using momentary assessment technology.

Poster presentation at the 39th Voorjaarscongres der Nederlandse Vereniging voor Psychiatrie (NVVP) in Amsterdam, the Netherlands, March 2011. Mechanisms of depression comorbidity in psychosis.

Poster presentation at the 39th Voorjaarscongres der Nederlandse Vereniging voor Psychiatrie (NVVP) in Amsterdam, the Netherlands, March 2011. Negative affect and paranoia in daily life.

Oral presentation at the $39^{\text {th }}$ Voorjaarscongres der Nederlandse Vereniging voor Psychiatrie (NVVP) in Amsterdam, the Netherlands, March 2011. Using ESM strategies as a therapeutic tool in depression.

Oral presentation at the Emotions Congres in Tilburg, the Netherlands, October 2011. Modification of positive affect in depression: the first therapeutic application of momentary assessment technology.

Oral presentation at the $40^{\text {th }}$ Voorjaarscongres der Nederlandse Vereniging voor Psychiatrie (NVVP) in Maastricht, the Netherlands, April 2012. Een eerste toepassing van ESM als therapeutisch hulpmiddel: het verbeteren van positief affect bij depressie 
Oral presentation at the $41^{\text {th }}$ Voorjaarscongres der Nederlandse Vereniging voor Psychiatrie (NVVP) in Maastricht, the Netherlands, April 2013. Gen-omgeving interactie bij psychose: hoe genen voor depressie het pad van trauma naar psychose beïnvloeden.

Oral presentation at the $2^{\text {nd }}$ Conference of the Society of Ambulatory Assessment in Amsterdam, the Netherlands, June 2013. Time-lagged moment-to-moment interplay between negative affect and paranoia: new insights in the affective pathway to psychosis.

Oral presentation at the Najaarscongres der Vereniging voor Gedragstherapie en Cognitieve Therapie in Veldhoven, the Netherlands, November 2013. Elektronische affect monitoring met feedbackinterventie in de behandeling van depressie: een gerandomiseerde gecontroleerde studie.

Oral presentation at the Sympopna congres: 'mHealth: voor de GGZ van morgen' in Utrecht, the Netherlands, September 2014. PsyMate en gepersonaliseerde feedback in de zorg. 
